\title{
One-loop Kubo estimations of the shear and bulk viscous coefficients for hot and magnetized bosonic and fermionic systems
}

\author{
Snigdha Ghosh $\oplus^{1, *}$ and Sabyasachi Ghosh $\odot^{2, \dagger}$ \\ ${ }^{1}$ Government General Degree College Kharagpur-II, \\ Madpur, Paschim Medinipur-721149, West Bengal, India \\ ${ }^{2}$ Indian Institute of Technology Bhilai, GEC Campus, \\ Sejbahar, Raipur-492015, Chhattisgarh, India
}

(Received 11 November 2020; accepted 16 April 2021; published 18 May 2021)

\begin{abstract}
The expressions of the shear viscosity and the bulk viscosity components in the presence of an arbitrary external magnetic field for a system of hot charged scalar bosons (spin 0) as well as for a system of hot charged Dirac fermions ( done by explicitly evaluating the thermomagnetic spectral functions of the energy-momentum tensors using the real time formalism of finite temperature field theory and the Schwinger proper time formalism. In the present work, a rich quantum field theoretical structure in the expressions of the viscous coefficients in nonzero magnetic field are found, which are different from their respective expressions obtained earlier via kinetic-theory-based calculations; though, in the absence of a magnetic field, the one-loop Kubo and the kinetic-theory-based expressions for the viscosities are known to be identical. We have identified that Kubo and kinetic-theory-based results of viscosity components follow a similar kind of temperature and magnetic field dependency. The relaxation time and the synchrotron frequency in the kinetic theory formalism are realized to be connected, respectively, with the thermal width of propagator and the transitions among the Landau levels of the charged particles in the Kubo formalism. We believe that the connection of the latter quantities is quite new and probably the present work is the first time addressing this interpretation along with the new expressions of viscosity components, not seen in existing works.
\end{abstract}

DOI: 10.1103/PhysRevD.103.096015

\section{INTRODUCTION}

The heavy ion collision experiment at relativistic energy can produce a superhot quark gluon plasma, which may be exposed under a strong magnetic field $B$ if the nucleusnucleus collision is noncentral. This magnetic field could be of the order of $\sim 10^{18} \mathrm{G}$ and comparable to the quantum chromodynamics $(\mathrm{QCD})$ scale $\left(e B \sim m_{\pi}^{2}\right.$ for RHIC-LHC energies) [1], for which many interesting QCD-linked phenomena [2-5] can be observed. Among a long list of interesting quantities, transport coefficients like shear viscosity and bulk viscosity are our aimed quantities in the present work. Owing to this fact, a long list of Refs. [6-34] have focused on the microscopic calculation of the transport coefficients, like the shear viscosity in Refs. [6-17], bulk viscosity in Refs. [18-21] and electrical conductivity in

\footnotetext{
*snigdha.physics@gmail.com

†sabyaphy@gmail.com
}

Published by the American Physical Society under the terms of the Creative Commons Attribution 4.0 International license. Further distribution of this work must maintain attribution to the author(s) and the published article's title, journal citation, and DOI. Funded by SCOAP.
Refs. [22-34] for the hot and/or dense QCD matter in the presence of a magnetic field. If we analyze the frameworks of those microscopic calculations, they are mostly in the kinetic-theory-based approaches.

For the shear viscosity in the presence of an external magnetic field, one can get five independent traceless tensors, with which five shear viscosity components will be linked, while a single traceless tensor with isotropic shear viscosity is found in the $B=0$ case. There are two possible sets of these five independent traceless tensors as proposed in Refs. [35,36], respectively. Using the former set, proposed in Ref. [35], the authors of Refs. [6,8-12,17] have obtained five shear viscosity components $\tilde{\eta}_{n}(n=0,1,2,3,4)$; while, using the latter set proposed in Ref. [36], the authors of Refs. $[14,15]$ have obtained their five shear viscosity components $\eta_{n}$. However, the $\tilde{\eta}_{n}$ 's and $\eta_{n}$ 's are interconnected and ultimately can be expressed in terms of the parallel, perpendicular and Hall components [10]. The general expressions of $\tilde{\eta}_{n}$ are obtained in the relaxation time approximation (RTA) of kinetic theory approach in Refs. $[8,10-12,17]$ and the same using the strong magnetic field approximation in Refs. [6,9]. In Ref. [14], the authors have obtained $\eta_{n}$ in RTAbased moment methods but its RTA-based kinetic theory calculation can be seen in Ref. [10]. 
However, a quantum field theoretical treatment at finite temperature and magnetic field via the Kubo relations has never been attempted in details which could reveal rich quantum structure in shear and bulk viscosity components. Though, in Ref. [15], the Kubo-type correlation structure has been considered but it is explored through a box simulation, not through a field theoretical calculation. So as far as our best knowledge, we are going to address for the first time a one-loop Kubo expression of viscosity components in the presence of a magnetic field by using a real time formalism of finite temperature field theory and the Schwinger proper time framework.

Let us revisit quickly the Kubo expression of shear viscosity of any bosonic or fermionic medium in the absence of a magnetic field [37-42]. Owing to the Kubo relation, shear viscosity can be related to the static limit (zero four-momentum limit) of the two-point correlation functions of the local viscous stress tensor $\pi^{\mu \nu}$. The simplest Feynman diagrams from the bosonic and fermion free Lagrangian (densities) will be boson-boson or fermionfermion loops, whose propagators must carry finite thermal width $\Gamma$. Without this $\Gamma$, one cannot get any nondivergent values; so this imposition of finite $\Gamma$ brings a quasiparticle picture, where the $\Gamma$ can be estimated from the interaction Lagrangian of a particular system and the numerical value of the shear viscosity of that system is mainly controlled by the strength of $\Gamma$. Interestingly, this one-loop Kubo expression [38-42] of shear viscosity becomes exactly identical to the RTA expression [43,44] with the relaxation time $\tau_{c}=1 / \Gamma$ in the absence of a magnetic field.

Now, at finite magnetic field, one should not expect the same expressions of viscosity components from the Kubo framework and the RTA framework $[6,8-12,14,17]$. The present work is going to reveal a difference between the expressions of the viscosities between the two frameworks at finite magnetic field picture. Here, we have found a rich quantum structure in the expressions of viscous coefficients, which might (not) be obtained by appropriate quantum extension of RTA frameworks. Reference [13] for shear viscosity and Refs. [22-24,32,32,33] for electrical conductivity calculations have followed the Landau quantization version of RTA. However, our Kubo expressions will have additional structures which are not the mere Landau quantized version of the corresponding RTA expressions of the viscosities. In the present work, we have considered two different systems-(i) system of charged scalar bosons (spin 0) and (ii) system of charged Dirac fermions ( $\operatorname{spin} \frac{1}{2}$ ) - and have calculated the corresponding thermomagnetic spectral functions of the energymomentum tensors (EMTs). The spectral function is the imaginary part of the Fourier transform of the local EMTEMT two-point correlator which is obtained using the real time formalism of finite temperature field theory and the Schwinger proper time formalism to incorporate the effects of finite temperature and external magnetic field, respectively. Then viscous coefficients are estimated from the thermomagnetic spectral functions using the Kubo relations in the covariant tensor basis of Ref. [36].

The article is organized as follows. We first start with the calculation of the in-medium spectral function of the energy-momentum tensor at zero magnetic field in Sec. II and at nonzero magnetic field in Sec. III. Next, Sec. IV has demonstrated how to obtain the shear and bulk viscosity from those spectral functions using the Kubo relations. After getting the new expressions for viscosity components, their numerical outcomes have been sketched and been tried to interpret in Sec. V. This is followed by Sec. VI where we have summarized the investigation. To compensate the calculation gaps, we have provided detailed Appendixes at the end.

\section{THE SPECTRAL FUNCTION OF THE ENERGY-MOMENTUM TENSOR}

The key microscopic quantity that is required to calculate the viscous coefficients of a thermal medium using the Kubo formalism [37] is the in-medium spectral function $\rho^{\mu \nu \alpha \beta}(q)$, given by

$$
\rho^{\mu \nu \alpha \beta}(q)=\operatorname{Im} i \int d^{4} x e^{i q \cdot x}\left\langle T^{\mu \nu}(x) T^{\alpha \beta}(0)\right\rangle_{R},
$$

where $T^{\mu \nu}(x)$ is the local EMT and $\langle\cdots\rangle_{R}$ represents the ensemble average of the retarded two-point correlation function. We will be using a metric tensor with signature $g^{\mu \nu}=\operatorname{diag}(1,-1,-1,-1)$. In order to use field theoretic methods, it is more convenient to express the spectral function in terms of time-ordered correlator as $[45,46]$

$\rho^{\mu \nu \alpha \beta}(q)=\tanh \left(\frac{q^{0}}{2 T}\right) \operatorname{Im} i \int d^{4} x e^{i q \cdot x}\left\langle\mathcal{T}_{C} T^{\mu \nu}(x) T^{\alpha \beta}(0)\right\rangle_{11}$,

where $\mathcal{T}_{C}$ is the time ordering with respect to the symmetric Schwinger-Keldysh contour $C$ in the complex time plane shown in Fig. 1 as used in the real time formalism (RTF) of finite temperature field theory. The subscript 11 in the above equation implies that the two points are on the real horizontal segment "(1)" of the contour $C$.

The form of the local EMT $T^{\mu \nu}(x)$ appearing in Eq. (2) depends on the particular system considered. In this work, we will be mainly considering two systems: (i) system of charged scalar bosons $B^{ \pm}$(spin 0) and (ii) system of charged Dirac fermions $F^{ \pm}$( $\operatorname{spin} \frac{1}{2}$ ). They are, respectively, described by the fields $\phi(x)$ and $\psi(x)$ which construct the following free Lagrangian (densities) [47]:

$$
\mathcal{L}_{\text {scalar }}=\partial^{\mu} \phi^{\dagger} \partial_{\mu} \phi-m^{2} \phi^{\dagger} \phi
$$




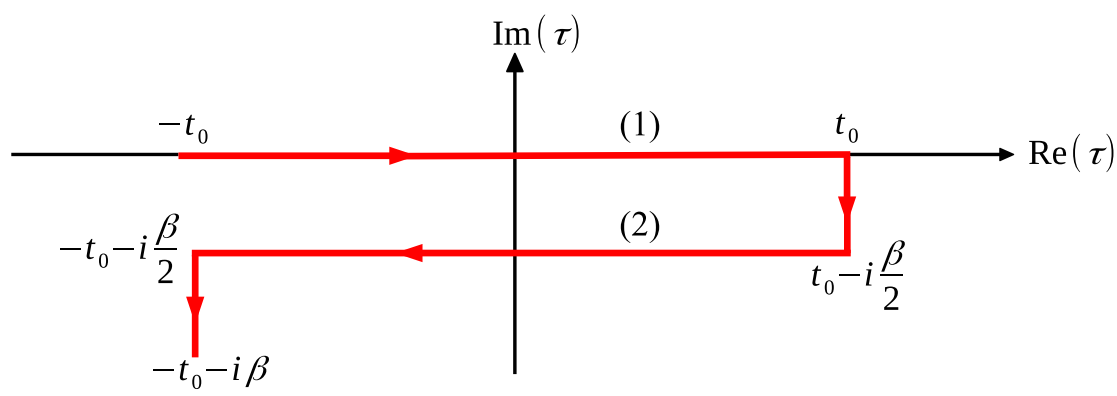

FIG. 1. The symmetric Schwinger-Keldysh contour $C$ in the complex time plane used in the RTF with $t_{0} \rightarrow \infty$ and $\beta=1 / T$. The two horizontal segments of the contour are referred as labels (1) and (2), respectively.

$$
\mathcal{L}_{\text {Dirac }}=\frac{i}{2}\left(\bar{\psi} \gamma^{\mu} \partial_{\mu} \psi-\partial_{\mu} \bar{\psi} \gamma^{\mu} \psi\right)-m \bar{\psi} \psi
$$

in which $m$ is the mass of the particles. Symmetric EMTs can be constructed out of the above Lagrangians as [47-49]

$$
T_{\text {scalar }}^{\mu \nu}=\partial^{\mu} \phi^{\dagger} \partial^{\nu} \phi-\frac{1}{2} g^{\mu \nu} \mathcal{L}_{\text {scalar }}+(\mu \leftrightarrow \nu)
$$

$$
\begin{aligned}
T_{\text {Dirac }}^{\mu \nu}= & \frac{i}{4}\left(\bar{\psi} \gamma^{\mu} \partial^{\nu} \psi-\partial^{\nu} \bar{\psi} \gamma^{\mu} \psi\right)-\frac{1}{2} g^{\mu \nu} \mathcal{L}_{\text {Dirac }} \\
& +(\mu \leftrightarrow \nu) .
\end{aligned}
$$

Using Eqs. (5) and (6), we now calculate the EMT correlation function $\left\langle\mathcal{T}_{C} T^{\mu \nu}(x) T^{\alpha \beta}(0)\right\rangle_{11}$ appearing in Eq. (2) for both the scalar and Dirac fields. The calculation have been provided in Appendix $\mathrm{A}$ and we get from Eqs. (A6) and (A15)

$$
\begin{aligned}
& \left\langle\mathcal{T}_{C} T_{\text {scalar }}^{\mu \nu}(x) T_{\text {scalar }}^{\alpha \beta}(0)\right\rangle_{11}=-\iint \frac{d^{4} p}{(2 \pi)^{4}} \frac{d^{4} k}{(2 \pi)^{4}} e^{-i x \cdot(p-k)} D_{11}(p ; m) D_{11}(k ; m) \mathcal{N}_{\text {scalar }}^{\mu \nu \alpha \beta}(k, p), \\
& \left\langle\mathcal{T}_{C} T_{\text {Dirac }}^{\mu \nu}(x) T_{\text {Dirac }}^{\alpha \beta}(0)\right\rangle_{11}=-\iint \frac{d^{4} p}{(2 \pi)^{4}} \frac{d^{4} k}{(2 \pi)^{4}} e^{-i x \cdot(p-k)} \tilde{D}_{11}(p ; m) \tilde{D}_{11}(k ; m) \mathcal{N}_{\text {Dirac }}^{\mu \nu \alpha \beta}(k, p),
\end{aligned}
$$

where $D_{11}, \tilde{D}_{11}$ and $\mathcal{N}_{\text {scalar,Dirac }}^{\mu \nu \alpha \beta}$ can be, respectively, read off from Eqs. (A5), (A16), (A7) and (A17). We now substitute the EMT correlators of Eqs. (7) and (8) into Eq. (2) and perform the $d^{4} x$ integral which yields the Dirac delta function $\delta^{4}(q-p+k)$. The Dirac delta function is in turn used to perform the $d^{4} p$ integral and the spectral function of EMT becomes

$$
\begin{aligned}
& \rho_{\text {scalar }}^{\mu \nu \alpha \beta}(q)=-\tanh \left(\frac{q^{0}}{2 T}\right) \operatorname{Im} i \int \frac{d^{4} k}{(2 \pi)^{4}} D_{11}(k ; m) D_{11}(p=q+k ; m) \mathcal{N}_{\text {scalar }}^{\mu \nu \alpha \beta}(k, p=q+k), \\
& \rho_{\text {Dirac }}^{\mu \nu \alpha \beta}(q)=-\tanh \left(\frac{q^{0}}{2 T}\right) \operatorname{Im} i \int \frac{d^{4} k}{(2 \pi)^{4}} \tilde{D}_{11}(k ; m) \tilde{D}_{11}(p=q+k ; m) \mathcal{N}_{\text {Dirac }}^{\mu \nu \alpha \beta}(k, p=q+k) .
\end{aligned}
$$

Substituting $D_{11}$ and $\tilde{D}_{11}$ from Eqs. (A5) and (A16) into the above two equations followed by performing the $d k^{0}$ integral, we get after some simplifications

$$
\begin{aligned}
\rho^{\mu \nu \alpha \beta}(q)= & \tanh \left(\frac{q^{0}}{2 T}\right) \pi \int \frac{d^{3} k}{(2 \pi)^{3}} \frac{1}{4 \omega_{k} \omega_{p}}\left[\{ 1 + a f _ { a } ( \omega _ { k } ) + a f _ { a } ( \omega _ { p } ) + 2 f _ { a } ( \omega _ { k } ) f _ { a } ( \omega _ { p } ) \} \left\{N^{\mu \nu \alpha \beta}\left(k^{0}=\omega_{k}\right) \delta\left(q^{0}-\omega_{k}-\omega_{p}\right)\right.\right. \\
& \left.+N^{\mu \nu \alpha \beta}\left(k^{0}=-\omega_{k}\right) \delta\left(q^{0}+\omega_{k}+\omega_{p}\right)\right\}+\left\{a f_{a}\left(\omega_{k}\right)+a f_{a}\left(\omega_{p}\right)+2 f_{a}\left(\omega_{k}\right) f_{a}\left(\omega_{p}\right)\right\} \\
& \left.\times\left\{N^{\mu \nu \alpha \beta}\left(k^{0}=-\omega_{k}\right) \delta\left(q^{0}-\omega_{k}+\omega_{p}\right)+N^{\mu \nu \alpha \beta}\left(k^{0}=\omega_{k}\right) \delta\left(q^{0}+\omega_{k}-\omega_{p}\right)\right\}\right],
\end{aligned}
$$

where $\omega_{k}=\sqrt{\vec{k}^{2}+m^{2}}, \omega_{p}=\sqrt{(\vec{k}+\vec{p})^{2}+m^{2}}$ and $f_{a}(x)=\frac{1}{e^{x / T}-a}$ with 


$$
a= \begin{cases}1 & \text { for scalar } \\ -1 & \text { for Dirac }\end{cases}
$$

Above Eq. (11) carries four Dirac delta functions which will give rise to the branch cuts of the spectral function in the complex $q^{0}$ plane. The kinematic regions where these Dirac delta functions are nonzero are, respectively, (i) $\sqrt{\vec{q}^{2}+4 m^{2}}<q_{0}<\infty$, (ii) $-\infty<q_{0}<-\sqrt{\vec{q}^{2}+4 m^{2}}$, (iii) and (iv) $\left|q^{0}\right|<|\vec{q}|$ as they appear in Eq. (11). Regions (i) and (ii) are, respectively, called the unitary-I and unitary-II cuts whereas regions (iii) and (iv) are called the Landau-II and Landau-I cuts, respectively $[38,46]$. For the evaluation of the viscous coefficients, we need to take the static limit $\vec{q}=\overrightarrow{0}, q^{0} \rightarrow 0$ of the EMT spectral function for which the unitary cuts do not contribute. Considering the Landau cuts only, we are left with

$$
\begin{gathered}
\rho^{\mu \nu \alpha \beta}\left(q^{0}, \vec{q}=\overrightarrow{0}\right)=\tanh \left(\frac{q^{0}}{2 T}\right) \pi \int \frac{d^{3} k}{(2 \pi)^{3}} \frac{1}{2 \omega_{k}^{2}} \delta\left(q^{0}\right) f_{a}\left(\omega_{k}\right)\left\{a+f_{a}\left(\omega_{k}\right)\right\}\left\{N^{\mu \nu \alpha \beta}\left(k^{0}=-\omega_{k}\right)+N^{\mu \nu \alpha \beta}\left(k^{0}=\omega_{k}\right)\right\} \\
=\lim _{\Gamma \rightarrow 0} \tanh \left(\frac{q^{0}}{2 T}\right) \int \frac{d^{3} k}{(2 \pi)^{3}} \frac{1}{2 \omega_{k}^{2}} \frac{\Gamma}{q_{0}^{2}+\Gamma^{2}} f_{a}\left(\omega_{k}\right)\left\{a+f_{a}\left(\omega_{k}\right)\right\}\left\{N^{\mu \nu \alpha \beta}\left(k^{0}=-\omega_{k}\right)+N^{\mu \nu \alpha \beta}\left(k^{0}=\omega_{k}\right)\right\},
\end{gathered}
$$

where a Breit-Wigner representation of the Dirac delta function has been used. According to the definition of Kubo relation, the dissipation coefficients are related to the zero four-momentum limit of $\rho^{\mu \nu \alpha \beta} / q_{0}$ or $\mathcal{S}^{\mu \nu \alpha \beta}=\frac{\partial \rho^{\mu \nu \alpha \beta}}{\partial q^{0}}$, owing to the L'Hospital's rule. Differentiating the spectral function with respect to $q^{0}$ and taking limit $q^{0} \rightarrow 0$, we finally obtain

$$
\mathcal{S}^{\mu \nu \alpha \beta}=\left.\frac{\partial \rho^{\mu \nu \alpha \beta}}{\partial q^{0}}\right|_{\vec{q}=\overrightarrow{0}, q^{0} \rightarrow 0}=\lim _{\Gamma \rightarrow 0} \frac{1}{T} \int \frac{d^{3} k}{(2 \pi)^{3}} \frac{1}{4 \omega_{k}^{2} \Gamma} f_{a}\left(\omega_{k}\right)\left\{a+f_{a}\left(\omega_{k}\right)\right\}\left[\left.N^{\mu \nu \alpha \beta}(k, k)\right|_{k^{0}=\omega_{k}}+\left.N^{\mu \nu \alpha \beta}(k, k)\right|_{k^{0}=-\omega_{k}}\right],
$$

where the simplified expressions of $N_{\text {scalar,Dirac }}^{\mu \nu \alpha \beta}(k)$ can be obtained from Eqs. (A8) and (A18) as

$$
\begin{aligned}
\mathcal{N}_{\text {scalar }}^{\mu \nu \alpha \beta}(k, k)= & 4 k^{\mu} k^{\nu} k^{\alpha} k^{\beta}-2\left(k^{2}-m^{2}\right)\left(g^{\mu \nu} k^{\alpha} k^{\beta}+g^{\alpha \beta} k^{\mu} k^{\nu}\right)+\left(k^{2}-m^{2}\right)^{2} g^{\mu \nu} g^{\alpha \beta}, \\
\mathcal{N}_{\text {Dirac }}^{\mu \nu \alpha \beta}(k, k)= & -8 k^{\mu} k^{\nu} k^{\alpha} k^{\beta}+\left(k^{2}-m^{2}\right)\left\{g^{\mu \alpha} k^{\nu} k^{\beta}+g^{\nu \alpha} k^{\mu} k^{\beta}+g^{\mu \beta} k^{\nu} k^{\alpha}+g^{\nu \beta} k^{\mu} k^{\alpha}\right. \\
& \left.+4 g^{\mu \nu} k^{\alpha} k^{\beta}+4 g^{\alpha \beta} k^{\mu} k^{\nu}\right\}-4\left(k^{2}-m^{2}\right)^{2} g^{\mu \nu} g^{\alpha \beta} .
\end{aligned}
$$

To get a nondivergent contribution of EMT spectral function in the zero momentum limit, further calculation will have to be continued with a finite value of $\Gamma$. This is the place where the interaction picture is introduced, which should be entered for a dissipative system. This $\Gamma$ can be identified as the thermal width or collision rate of the constituent particles, and it reciprocally measures the dissipative coefficients, like the shear viscosity and the bulk viscosity.

It may be noted that, in the present method, we have introduced the interaction information $\Gamma$ via the transformation of Dirac delta functions to Breit-Wigner functions, from which the noninteracting picture is realized as $\Gamma \rightarrow 0$ limit. This similar kind of transformation from noninteracting $(\Gamma=0)$ to interacting $(\Gamma \neq 0)$ picture can also be done by introducing $i \Gamma / 2$ in the propagators, located in our one-loop diagrammatic representation of the transport coefficients.

\section{THE SPECTRAL FUNCTION OF THE EMT IN THE PRESENCE OF AN EXTERNAL MAGNETIC FIELD}

In the presence of an external electromagnetic field described by the four-potential $A_{\text {ext }}^{\mu}(x)$, the Lagrangians of Eqs. (3) and (4) are modified to [50]

$$
\begin{gathered}
\mathcal{L}_{\text {scalar }}=D^{* \mu} \phi^{\dagger} D_{\mu} \phi-m^{2} \phi^{\dagger} \phi, \\
\mathcal{L}_{\text {Dirac }}=\frac{i}{2}\left(\bar{\psi} \gamma^{\mu} D_{\mu} \psi-D^{* \mu} \bar{\psi} \gamma^{\mu} \psi\right)-m \bar{\psi} \psi,
\end{gathered}
$$

where $D^{\mu}=\partial^{\mu}+i e A_{\text {ext }}^{\mu}(x)$ and $D^{* \mu}=\partial^{\mu}-i e A_{\text {ext }}^{\mu}(x)$ are the covariant derivatives incorporating the minimal coupling between the charged particle with charge $e$ (we consider $e>0$ ) and the electromagnetic field. The symmetric EMT in the presence of an electromagnetic field now becomes $[47,50]$ 


$$
\begin{gathered}
T_{\text {scalar }}^{\mu \nu}=D^{* \mu} \phi^{\dagger} D^{\nu} \phi-\frac{1}{2} g^{\mu \nu} \mathcal{L}_{\text {scalar }}+(\mu \leftrightarrow \nu), \\
T_{\text {Dirac }}^{\mu \nu}=\frac{i}{4}\left(\bar{\psi} \gamma^{\mu} D^{\nu} \psi-D^{* \nu} \bar{\psi} \gamma^{\mu} \psi\right)-\frac{1}{2} g^{\mu \nu} \mathcal{L}_{\text {Dirac }}+(\mu \leftrightarrow \nu) .
\end{gathered}
$$

Let us now consider a constant magnetic field $\vec{B}=B \hat{z}$ along the positive $\hat{z}$ direction. Using Eqs. (19) and (20), we now calculate the EMT correlation function $\left\langle\mathcal{T}_{C} T^{\mu \nu}(x) T^{\alpha \beta}(0)\right\rangle_{11}^{B}$ in the presence of an external magnetic field for both the scalar and Dirac field as sketched in Appendix B. We obtain from Eqs. (B11) and (B18)

$$
\begin{aligned}
& \left\langle\mathcal{T}_{C} T_{\text {scalar }}^{\mu \nu}(x) T_{\text {scalar }}^{\alpha \beta}(0)\right\rangle_{11}^{B}=-\iint \frac{d^{4} p}{(2 \pi)^{4}} \frac{d^{4} k}{(2 \pi)^{4}} e^{-i x \cdot(p-k)} \sum_{l=0}^{\infty} \sum_{n=0}^{\infty} D_{11}\left(p_{\|} ; m_{n}\right) D_{11}\left(k_{\|} ; m_{l}\right) \mathcal{N}_{l n ; \text { scalar }}^{\mu \nu \alpha \beta}(k, p), \\
& \left\langle\mathcal{T}_{C} T_{\text {Dirac }}^{\mu \nu}(x) T_{\text {Dirac }}^{\alpha \beta}(0)\right\rangle_{11}^{B}=-\iint \frac{d^{4} p}{(2 \pi)^{4}} \frac{d^{4} k}{(2 \pi)^{4}} e^{-i x \cdot(p-k)} \sum_{l=0}^{\infty} \sum_{n=0}^{\infty} \tilde{D}_{11}\left(p_{\|} ; m_{n}\right) \tilde{D}_{11}\left(k_{\|} ; m_{l}\right) \mathcal{N}_{l n ; \text { Dirac }}^{\mu \nu \alpha \beta}(k, p),
\end{aligned}
$$

where $D_{11}, \tilde{D}_{11}$ and $\mathcal{N}_{l n \text {;scalar,Dirac }}^{\mu \nu \alpha}$ can be, respectively, read off from Eqs. (A5), (A16), (B12) and (B19) and $m_{l}=$ $\sqrt{m^{2}+(2 l+1-2 s) e B}$ with $s$ being the spin of the particle $(s=0$ for the scalar and $s=1 / 2$ for the Dirac).

Similar to the zero magnetic field case, we now substitute the EMT correlators of Eqs. (21) and (22) into Eq. (2) and perform the $d^{4} x$ integral which yields the Dirac delta function $\delta^{4}(q-p+k)$. The Dirac delta function is in turn used to perform the $d^{4} p$ integral and the thermomagnetic spectral function of EMT becomes

$$
\begin{aligned}
& \rho_{\text {scalar }}^{\mu \nu \alpha \beta}(q)=-\tanh \left(\frac{q^{0}}{2 T}\right) \operatorname{Im} i \int \frac{d^{4} k}{(2 \pi)^{4}} \sum_{l=0}^{\infty} \sum_{n=0}^{\infty} D_{11}\left(k_{\|} ; m_{l}\right) D_{11}\left(p_{\|}=q_{\|}+k_{\|} ; m_{n}\right) \mathcal{N}_{l n ; \text { scalar }}^{\mu \nu \alpha \beta}(k, p=q+k), \\
& \rho_{\text {Dirac }}^{\mu \nu \alpha \beta}(q)=-\tanh \left(\frac{q^{0}}{2 T}\right) \operatorname{Im} i \int \frac{d^{4} k}{(2 \pi)^{4}} \sum_{l=0}^{\infty} \sum_{n=0}^{\infty} \tilde{D}_{11}\left(k_{\|} ; m_{l}\right) \tilde{D}_{11}\left(p_{\|}=q_{\|}+k_{\|} ; m_{n}\right) \mathcal{N}_{l n ; \text { Dirac }}^{\mu \nu \alpha \beta}(k, p=q+k) .
\end{aligned}
$$

Substituting $D_{11}$ and $\tilde{D}_{11}$ from Eqs. (A5) and (A16) into the above two equations followed by performing the $d k^{0}$ integral, we get after some simplifications

$$
\begin{aligned}
\rho^{\mu \nu \alpha \beta}(q)= & \tanh \left(\frac{q^{0}}{2 T}\right) \pi \sum_{l=0}^{\infty} \sum_{n=0}^{\infty} \int \frac{d^{3} k}{(2 \pi)^{3}} \frac{1}{4 \omega_{k l} \omega_{p n}}\left[\left\{1+a f_{a}\left(\omega_{k l}\right)+a f_{a}\left(\omega_{p n}\right)+2 f_{a}\left(\omega_{k l}\right) f_{a}\left(\omega_{p n}\right)\right\}\right. \\
& \times\left\{N_{l n}^{\mu \nu \alpha \beta}\left(k^{0}=\omega_{k l}\right) \delta\left(q^{0}-\omega_{k l}-\omega_{p n}\right)+N_{l n}^{\mu \nu \alpha \beta}\left(k^{0}=-\omega_{k l}\right) \delta\left(q^{0}+\omega_{k l}+\omega_{p n}\right)\right\} \\
& +\left\{a f_{a}\left(\omega_{k l}\right)+a f_{a}\left(\omega_{p n}\right)+2 f_{a}\left(\omega_{k l}\right) f_{a}\left(\omega_{p n}\right)\right\}\left\{N_{l n}^{\mu \nu \alpha \beta}\left(k^{0}=-\omega_{k l}\right) \delta\left(q^{0}-\omega_{k l}+\omega_{p n}\right)\right. \\
& \left.\left.+N_{l n}^{\mu \nu \alpha \beta}\left(k^{0}=\omega_{k l}\right) \delta\left(q^{0}+\omega_{k l}-\omega_{p n}\right)\right\}\right],
\end{aligned}
$$

where $\quad \omega_{k l}=\sqrt{k_{z}^{2}+m_{l}^{2}} \quad$ and $\quad \omega_{p n}=\sqrt{p_{z}^{2}+m_{n}^{2}}=$ $\sqrt{\left(k_{z}+q_{z}\right)^{2}+m_{n}^{2}}$. Similar to the zero field case, the spectral function in the presence of an external magnetic field contains four Dirac delta functions giving rise to branch cuts of the spectral function in the complex energy plane. The kinematic regions where these Dirac delta functions are nonzero now depends on the Landau levels of the charged particles as well. Thus, when summed over an infinite number of Landau levels, the kinematic regions for the unitary-I and unitary-II cuts comes out to be $\sqrt{q_{z}^{2}+4\left(m^{2}+e B\right)}<q^{0}<\infty$ and $-\infty<q^{0}<$ $-\sqrt{q_{z}^{2}+4\left(m^{2}+e B\right)}$, respectively, whereas the kinematic domain for both the Landau cuts becomes $\left|q^{0}\right|<$ $\sqrt{q_{z}^{2}+\left(\sqrt{m^{2}+e B}-\sqrt{m^{2}+3 e B}\right)^{2}}$ for the scalar case. On the other hand, for the Dirac case, the correspond kinematic domains for unitary-I and unitary-II cuts are $\sqrt{q_{z}^{2}+4 m^{2}}<q^{0}<\infty$ and $-\infty<q^{0}<-\sqrt{q_{z}^{2}+4 m^{2}}$, respectively, whereas the same for both the Landau cuts is $\left|q^{0}\right|<\sqrt{q_{z}^{2}+\left(m-\sqrt{m^{2}+2 e B}\right)^{2}}[51-53]$.

As already discussed in Sec. II, for the evaluation of the viscous coefficients, we only need the Landau cuts and the spectral function becomes 


$$
\begin{aligned}
\rho^{\mu \nu \alpha \beta}\left(q^{0}, \vec{q}=\overrightarrow{0}\right)= & \tanh \left(\frac{q^{0}}{2 T}\right) \pi \sum_{l=0}^{\infty} \sum_{n=0}^{\infty} \int \frac{d^{3} k}{(2 \pi)^{3}} \frac{1}{4 \omega_{k l} \omega_{k n}}\left\{a f_{a}\left(\omega_{k l}\right)+a f_{a}\left(\omega_{k n}\right)+2 f_{a}\left(\omega_{k l}\right) f_{a}\left(\omega_{k n}\right)\right\} \\
& \times\left\{N_{l n}^{\mu \nu \alpha \beta}\left(k^{0}=-\omega_{k l}\right) \delta\left(q^{0}-\omega_{k l}+\omega_{k n}\right)+N_{l n}^{\mu \nu \alpha \beta}\left(k^{0}=\omega_{k l}\right) \delta\left(q^{0}+\omega_{k l}-\omega_{k n}\right)\right\} \\
= & \lim _{\Gamma \rightarrow 0} \tanh \left(\frac{q^{0}}{2 T}\right) \sum_{l=0}^{\infty} \sum_{n=0}^{\infty} \int \frac{d^{3} k}{(2 \pi)^{3}} \frac{1}{4 \omega_{k l} \omega_{k n}}\left\{a f_{a}\left(\omega_{k l}\right)+a f_{a}\left(\omega_{k n}\right)+2 f_{a}\left(\omega_{k l}\right) f_{a}\left(\omega_{k n}\right)\right\} \\
& \times\left\{N_{l n}^{\mu \nu \alpha \beta}\left(k^{0}=-\omega_{k l}\right) \frac{\Gamma}{\left(q^{0}-\omega_{k l}+\omega_{k n}\right)^{2}+\Gamma^{2}}+N_{l n}^{\mu \nu \alpha \beta}\left(k^{0}=\omega_{k l}\right) \frac{\Gamma}{\left(q^{0}+\omega_{k l}-\omega_{k n}\right)^{2}+\Gamma^{2}}\right\},
\end{aligned}
$$

where a Breit-Wigner representation of the Dirac delta function has again been used. Differentiating the above equation with respect to $q^{0}$ and taking limit $q^{0} \rightarrow 0$, we finally obtain

$$
\begin{aligned}
\mathcal{S}^{\mu \nu \alpha \beta}= & \lim _{\Gamma \rightarrow 0} \sum_{l=0}^{\infty} \sum_{n=0}^{\infty} \frac{1}{2 T} \int \frac{d^{3} k}{(2 \pi)^{3}} \frac{1}{4 \omega_{k l} \omega_{k n}} \frac{\Gamma}{\left(\omega_{k l}-\omega_{k n}\right)^{2}+\Gamma^{2}}\left\{a f_{a}\left(\omega_{k l}\right)+a f_{a}\left(\omega_{k n}\right)+2 f_{a}\left(\omega_{k l}\right) f_{a}\left(\omega_{k n}\right)\right\} \\
& \times\left[\left.N_{l n}^{\mu \nu \alpha \beta}(k, k)\right|_{k^{0}=\omega_{k l}}+\left.N_{l n}^{\mu \nu \alpha \beta}(k, k)\right|_{k^{0}=-\omega_{k l}}\right],
\end{aligned}
$$

where the simplified expressions of $N_{l n ; \text {;calar,Dirac }}^{\mu \nu \alpha \beta}(k, k)$ can be obtained from Eqs. (B13) and (B28) as

$$
\mathcal{N}_{l n ; \text { scalar }}^{\mu \nu \alpha \beta}(k, k)=4 \mathcal{A}_{l n}\left(k_{\perp}^{2}\right)\left\{4 k^{\mu} k^{\nu} k^{\alpha} k^{\beta}-2\left(k^{2}-m^{2}\right)\left(g^{\mu \nu} k^{\alpha} k^{\beta}+g^{\alpha \beta} k^{\mu} k^{\nu}\right)+\left(k^{2}-m^{2}\right)^{2} g^{\mu \nu} g^{\alpha \beta}\right\}
$$

and

$$
\begin{aligned}
\mathcal{N}_{l n ; \text { Dirac }}^{\mu \nu \alpha \beta}(k, k)= & -16 \mathcal{B}_{l n}\left(k_{\perp}^{2}\right)\left[k^{\nu} k^{\beta}\left(2 k_{\perp}^{\mu} k_{\perp}^{\alpha}-k_{\perp}^{2} g^{\mu \alpha}\right)-g^{\mu \nu} k^{\beta} k_{\perp}^{2}\left(k_{\perp}^{\alpha}-k_{\|}^{\alpha}\right)-g^{\alpha \beta} k^{\nu} k_{\perp}^{2}\left(k_{\perp}^{\mu}-k_{\|}^{\mu}\right)\right. \\
& \left.+g^{\mu \nu} g^{\alpha \beta} k_{\perp}^{2}\left(k_{\perp}^{2}-k_{\|}^{2}+m^{2}\right)\right]-2 \mathcal{C}_{l n}\left(k_{\perp}^{2}\right)\left[k^{\nu} k^{\beta}\left\{2 k_{\|}^{\mu} k_{\|}^{\alpha}-\left(k_{\|}^{2}-m^{2}\right) g_{\|}^{\mu \alpha}\right\}-\left(k_{\|}^{2}-m^{2}\right) g^{\mu \nu} k^{\beta} k_{\|}^{\alpha}\right. \\
& \left.-\left(k_{\|}^{2}-m^{2}\right) g^{\alpha \beta} k^{\nu} k_{\|}^{\mu}+g^{\mu \nu} g^{\alpha \beta}\left(k_{\|}^{2}-m^{2}\right)^{2}\right]-2 \mathcal{D}_{l n}\left(k_{\perp}^{2}\right)\left(k_{\|}^{2}-m^{2}\right)\left[k^{\nu} k^{\beta} g_{\perp}^{\mu \alpha}-g^{\mu \nu} k^{\beta} k_{\perp}^{\alpha}\right. \\
& \left.-g^{\alpha \beta} k^{\nu} k_{\perp}^{\mu}+g^{\mu \nu} g^{\alpha \beta} k_{\perp}^{2}\right]-4 \mathcal{E}_{l n}\left(k_{\perp}^{2}\right)\left[k^{\nu} k^{\beta}\left(k_{\|}^{\mu} k_{\perp}^{\alpha}+k_{\perp}^{\mu} k_{\|}^{\alpha}\right)-g^{\mu \nu} k^{\beta}\left\{\left(k_{\|}^{2}-m^{2}\right) k_{\perp}^{\alpha}+k_{\perp}^{2} k_{\|}^{\alpha}\right\}\right. \\
& \left.-g^{\alpha \beta} k^{\nu}\left\{\left(k_{\|}^{2}-m^{2}\right) k_{\perp}^{\mu}+k_{\perp}^{2} k_{\|}^{\mu}\right\}+2 g^{\mu \nu} g^{\alpha \beta} k_{\perp}^{2}\left(k_{\|}^{2}-m^{2}\right)\right]+(\mu \leftrightarrow \nu)+(\alpha \leftrightarrow \beta)+(\mu \leftrightarrow \nu, \alpha \leftrightarrow \beta),
\end{aligned}
$$

respectively, in which the functions $\mathcal{A}_{l n}\left(k_{\perp}^{2}\right), \mathcal{B}_{l n}\left(k_{\perp}^{2}\right), \ldots, \mathcal{E}_{l n}\left(k_{\perp}^{2}\right)$ are defined in Eqs. (B14) and (B24)-(B27).

A few comments on the Lorentz structure of the EMT spectral functions are in order here. In the current work, we have considered only the symmetric part of the EMT spectral function which is proportional to the imaginary part of the retarded correlator. On the other hand, the real part of the correlator contributing to the antisymmetric piece of the spectral function has been discarded. Another way of saying this is that, in Eqs. (23) and (24), if the tensor $\mathcal{N}_{l n}^{\mu \nu \alpha \beta}$ contains terms like $i \epsilon_{\perp}^{\mu \alpha} k^{\nu} k^{\beta}$, then Eq. (25) will in turn have additional terms related to the real part of the Fourier-transformed correlator [54]. In the current work, we did not get such antisymmetric terms contributing to the viscous coefficients at oneloop order.

\section{VISCOUS COEFFICIENTS FROM THE SPECTRAL FUNCTION IN KUBO FORMALISM}

Owing to the Kubo relation [37], the viscous coefficients (shear and bulk) can be calculated from the spectral functions of the EMT which have already been obtained in Secs. II and III. We will first revisit the $B=0$ case [38-42] before discussing the finite $B$ calculations.

In the absence of an external magnetic field, the shear viscosity $(\eta)$ and bulk viscosity $(\zeta)$ are obtained from [38]

$$
v=\mathcal{P}_{\mu \nu \alpha \beta}^{(v)} \mathcal{S}^{\mu \nu \alpha \beta} ; \quad v \in\{\eta, \zeta\},
$$

where 


$$
\begin{gathered}
\mathcal{P}_{\mu \nu \alpha \beta}^{(\eta)}=\frac{1}{10}\left(\Delta_{\mu}^{\sigma} \Delta_{\nu}^{\rho}-\frac{1}{3} \Delta^{\sigma \rho} \Delta_{\mu \nu}\right)\left(\Delta_{\sigma \alpha} \Delta_{\rho \beta}-\frac{1}{3} \Delta_{\sigma \rho} \Delta_{\alpha \beta}\right), \\
\mathcal{P}_{\mu \nu \alpha \beta}^{(\zeta)}=\left(\frac{1}{3} \Delta_{\mu \nu}+\theta u_{\mu} u_{\nu}\right)\left(\frac{1}{3} \Delta_{\alpha \beta}+\theta u_{\alpha} u_{\beta}\right)
\end{gathered}
$$

in which $\Delta^{\mu \nu}=\left(g^{\mu \nu}-u^{\mu} u^{\nu}\right), \theta=\left(\frac{\partial P}{\partial \varepsilon}\right), P$ is the pressure and $\varepsilon$ is the energy density of the system being considered. Substituting Eq. (14) into Eq. (31), we obtain the viscous coefficients at zero magnetic field as

$$
\begin{aligned}
v= & \frac{1}{T} \int \frac{d^{3} k}{(2 \pi)^{3}} \frac{1}{2 \omega_{k}^{2} \Gamma} f_{a}\left(\omega_{k}\right)\left\{a+f_{a}\left(\omega_{k}\right)\right\} \mathcal{N}^{(v)}(\vec{k}) ; \\
v & \in\{\eta, \zeta\},
\end{aligned}
$$

where

$$
\mathcal{N}^{(v)}(\vec{k})=\frac{1}{2} \mathcal{P}_{\mu \nu \alpha \beta}^{(v)}\left\{\left.\mathcal{N}^{\mu \nu \alpha \beta}(k, k)\right|_{k^{0}=\omega_{k}}+\left.\mathcal{N}^{\mu \nu \alpha \beta}(k, k)\right|_{k^{0}=-\omega_{k}}\right\} .
$$

Substituting Eqs. (15), (16), (32) and (33) into Eq. (35) and simplifying, we obtain

$$
\begin{aligned}
& \mathcal{N}_{\text {scalar }}^{(\eta)}=\frac{4}{15} \vec{k}^{4}, \quad \mathcal{N}_{\text {scalar }}^{(\zeta)}=\frac{4}{9}\left\{m^{2}+(3 \theta-1) \omega_{k}^{2}\right\}^{2}, \\
& \mathcal{N}_{\text {Dirac }}^{(\eta)}=-\frac{8}{15} \vec{k}^{4}, \quad \mathcal{N}_{\text {Dirac }}^{(\zeta)}=-\frac{8}{9}\left\{m^{2}+(3 \theta-1) \omega_{k}^{2}\right\}^{2} .
\end{aligned}
$$

It is now easy to check that substitution of Eqs. (36) and (37) into Eq. (34) yields the well-known expressions of the shear and bulk viscosities for the system of scalar bosons and system of Dirac fermions [38-42]:

$$
\begin{aligned}
\eta_{\text {scalar }}= & \frac{2}{15 T} \int \frac{d^{3} k}{(2 \pi)^{3}} \frac{\vec{k}^{4}}{\omega_{k}^{2} \Gamma} f\left(\omega_{k}\right)\left\{1+f\left(\omega_{k}\right)\right\}, \\
\eta_{\text {Dirac }}= & \frac{4}{15 T} \int \frac{d^{3} k}{(2 \pi)^{3}} \frac{\vec{k}^{4}}{\omega_{k}^{2} \Gamma} \tilde{f}\left(\omega_{k}\right)\left\{1-\tilde{f}\left(\omega_{k}\right)\right\}, \\
\zeta_{\text {scalar }}= & \frac{2}{9 T} \int \frac{d^{3} k}{(2 \pi)^{3}} \frac{1}{\omega_{k}^{2} \Gamma}\left\{m^{2}+(3 \theta-1) \omega_{k}^{2}\right\}^{2} \\
& \times f\left(\omega_{k}\right)\left\{1+f\left(\omega_{k}\right)\right\}, \\
\zeta_{\text {Dirac }}= & \frac{4}{9 T} \int \frac{d^{3} k}{(2 \pi)^{3}} \frac{1}{\omega_{k}^{2} \Gamma}\left\{m^{2}+(3 \theta-1) \omega_{k}^{2}\right\}^{2} \\
& \times \tilde{f}\left(\omega_{k}\right)\left\{1-\tilde{f}\left(\omega_{k}\right)\right\} .
\end{aligned}
$$

The above expressions of shear and bulk viscosity for scalar and Dirac system from the Kubo framework [38-42] are exactly identical to the same obtained using the RTA in kinetic theory formalism $[43,44]$.

Let us now switch on the external magnetic field. The main difference between $B=0$ and $B \neq 0$ pictures of shear and bulk viscosity will start from their macroscopic definition, where the viscosity coefficients have basically appeared as the proportionality constants between thermodynamical force tensors and gradient tensors. Unlike single gradient tensors for $\eta$ and $\zeta$ at $B=0$, one can get five (traceless) and two (nonzero trace) independent gradient tensors, for which five shear viscosity coefficients $\eta_{n}(n=0,1,2,3,4)$ and two bulk viscosity coefficients $\zeta_{\perp, \|}$ will be introduced in the $B \neq 0$ picture. As shown in Ref. [35] by Landau, in the presence of an external magnetic field, there will be seven viscous coefficients (in Landau's notation, they are $\eta, \zeta, \eta_{1}$, $\eta_{2}, \eta_{3}, \eta_{4}$ and $\left.\zeta_{1}\right)$. The viscosity coefficients appear as the expansion coefficients of the tensorial decomposition (in a particular basis) of the viscous stress tensor. However, the choice of the tensor basis to decompose the viscous stress tensor is not unique. Apart from Ref. [35], in Refs. [36,55], Huang et al. have constructed a different tensor basis and thus obtained the seven viscosity coefficients (denoted as $\eta_{0}, \eta_{1}$, $\eta_{2}, \eta_{3}, \eta_{4}, \zeta_{\|}$and $\zeta_{\perp}$ in their notation). In a more recent work [56], the authors have constructed a more general tensor basis and thus obtained seven independent transport coefficients namely the two electrical resistivities $\left(\rho_{\|}\right.$and $\left.\rho_{\perp}\right)$ and five viscosities $\left(\eta_{\|}, \eta_{\perp}, \zeta_{\|}, \zeta_{\perp}\right.$ and $\left.\zeta_{\times}=\zeta_{\times}^{\prime}\right)$. From a physical point of view, it is understood that the viscous coefficients defined in Refs. $[35,36,55,56]$ must be interconnected and they can be expressed in terms of one another. In the RTAbased kinetic theory or moment methods, Refs. [6,8-12,17] have used the tensor basis of Ref. [35] whereas Refs. [10,14] have used the tensor basis of Refs. [36,55]. In this work, we have used the tensor decomposition of Refs. [36,55] and thus obtained the seven viscosity coefficients namely $\eta_{0}, \eta_{1}$, $\eta_{2}, \eta_{3}, \eta_{4}, \zeta_{\|}$and $\zeta_{\perp}$ using the Kubo formalism. Exploring the same with the tensor basis of Ref. [35] as well as Ref. [56] might be a very interesting future project.

Let us start with connecting relations between viscous coefficients $v$ and spectral function [36,55]:

$$
\begin{aligned}
& v=-\xi^{(v)} \eta_{0}+\mathcal{P}_{\mu \nu \alpha \beta}^{(v)} \mathcal{S}^{\mu \nu \alpha \beta} ; \\
& v \in\left\{\eta_{0}, \eta_{1}, \eta_{2}, \eta_{3}, \eta_{4}, \zeta_{\perp}, \zeta_{\|}\right\},
\end{aligned}
$$

where

$$
\xi^{(v)}= \begin{cases}4 / 3 & \text { if } v=\eta_{1} \\ 1 & \text { if } v=\eta_{2} \\ 0 & \text { otherwise }\end{cases}
$$

and $\mathcal{P}_{\mu \nu \alpha \beta}^{(v)}$ are given by 


$$
\begin{gathered}
\mathcal{P}_{\mu \nu \alpha \beta}^{\left(\eta_{0}\right)}=\frac{1}{4}\left(\Xi_{\mu}^{\sigma} \Xi_{\nu}^{\rho}-\frac{1}{2} \Xi^{\sigma \rho} \Xi_{\mu \nu}\right)\left(\Xi_{\sigma \alpha} \Xi_{\rho_{\beta}}-\frac{1}{2} \Xi_{\sigma \rho} \Xi_{\alpha \beta}\right), \\
\mathcal{P}_{\mu \nu \alpha \beta}^{\left(\eta_{1}\right)}=2\left(b_{\mu} b_{\nu}-\theta u_{\mu} u_{\nu}\right)\left(\frac{1}{2} \Xi_{\alpha \beta}+(\theta+\phi) u_{\alpha} u_{\beta}\right), \\
\mathcal{P}_{\mu \nu \alpha \beta}^{\left(\eta_{2}\right)}=-\frac{1}{2} \Xi_{\mu}^{\sigma} b_{\nu} \Xi_{\sigma \alpha} b_{\alpha}, \\
\mathcal{P}_{\mu \nu \alpha \beta}^{\left(\eta_{3}\right)}=-\frac{1}{8}\left(\Xi_{\mu}^{\sigma} \Xi_{\nu}^{\rho}-\frac{1}{2} \Xi^{\sigma \rho} \Xi_{\mu \nu}\right) b_{\sigma}^{\lambda}\left(\Xi_{\lambda \alpha} \Xi_{\rho_{\beta}}-\frac{1}{2} \Xi_{\lambda \rho} \Xi_{\alpha \beta}\right), \\
\mathcal{P}_{\mu \nu \alpha \beta}^{\left(\eta_{4}\right)}=\frac{1}{2} b_{\rho \sigma} \Xi_{\mu}^{\rho} b_{\nu} \Xi_{\alpha}^{\sigma} b_{\beta},
\end{gathered}
$$$$
\mathcal{P}_{\mu \nu \alpha \beta}^{\left(\zeta_{\perp}\right)}=\frac{1}{3}\left(\Delta_{\mu \nu}+(3 \theta+2 \phi) u_{\mu} u_{\nu}\right)\left(\frac{1}{2} \Xi_{\alpha \beta}+(\theta+\phi) u_{\alpha} u_{\beta}\right),
$$$$
\mathcal{P}_{\mu \nu \alpha \beta}^{\left(\zeta_{\|}\right)}=-\frac{1}{3}\left(\Delta_{\mu \nu}+(\theta+2 \phi) u_{\mu} u_{\nu}\right)\left(b_{\alpha} b_{\beta}-\theta u_{\alpha} u_{\beta}\right),
$$

where $b^{\mu}=\frac{1}{2 B} \varepsilon^{\mu \nu \alpha \beta} F_{\nu \alpha} u_{\beta}, F^{\mu \nu}=\left(\partial^{\mu} A_{\text {ext }}^{\nu}-\partial^{\nu} A_{\text {ext }}^{\mu}\right)$ is the electromagnetic field strength tensor, $b^{\mu \nu}=\varepsilon^{\mu \nu \alpha \beta} b_{\alpha} u_{\beta}$, and $\Xi^{\mu \nu}=\Delta^{\mu \nu}+b^{\mu} b^{\nu}$ with the convention of the Levi-Civita tensor $\varepsilon^{0123}=1$. In the local rest frame (LRF), $b_{\mathrm{LRF}}^{\mu} \equiv$ $(0,0,0,1)$. In Eqs. (43)-(49), the thermodynamic quantities $\theta=\left(\frac{\partial P}{\partial \varepsilon}\right)_{B}$ and $\phi=-B\left(\frac{\partial M}{\partial \varepsilon}\right)_{B}$ in which $M$ is the magnetization of the medium.

Substituting Eq. (28) into Eq. (42), we obtain the viscous coefficients in the presence of a constant external magnetic field as

$$
v=\xi^{(v)} \eta_{0}+\sum_{l=0}^{\infty} \sum_{n=0}^{\infty} \frac{1}{T} \int \frac{d^{3} k}{(2 \pi)^{3}} \frac{1}{4 \omega_{k l} \omega_{k n}} \frac{\Gamma}{\left(\omega_{k l}-\omega_{k n}\right)^{2}+\Gamma^{2}}\left\{a f_{a}\left(\omega_{k l}\right)+a f_{a}\left(\omega_{k n}\right)+2 f_{a}\left(\omega_{k l}\right) f_{a}\left(\omega_{k n}\right)\right\} \mathcal{N}_{l n}^{(v)}(\vec{k}),
$$

where

$$
\mathcal{N}_{l n}^{(v)}(\vec{k})=\frac{1}{2} \mathcal{P}_{\mu \nu \alpha \beta}^{(v)}\left\{\left.\mathcal{N}_{l n}^{\mu \nu \alpha \beta}(k, k)\right|_{k^{0}=\omega_{k l}}+\left.\mathcal{N}_{l n}^{\mu \nu \alpha \beta}(k, k)\right|_{k^{0}=-\omega_{k l}}\right\}
$$

Substitution of Eqs. (29), (30), and (43)-(49) into Eq. (51) yields after bit simplifications the following final expressions of $\mathcal{N}_{l n}^{(v)}(\vec{k})$ :

$$
\begin{gathered}
\mathcal{N}_{l n ; \text { scalar }}^{\left(\eta_{0}\right)}(\vec{k})=2 \mathcal{A}_{l n}\left(k_{\perp}^{2}\right) k_{\perp}^{4}, \\
\mathcal{N}_{l n ; \text { scalar }}^{\left(\eta_{1}\right)}(\vec{k})=-8 \mathcal{A}_{l n}\left(k_{\perp}^{2}\right)\left\{(1-\theta) \omega_{k l}^{2}+(1+\theta) k_{\perp}^{2}+(1-\theta) k_{z}^{2}-(1+\theta) m^{2}\right\} \\
\times\left\{(1-\theta-\phi) \omega_{k l}^{2}+(\theta+\phi) k_{\perp}^{2}-(1+\theta+\phi)\left(k_{z}^{2}+m^{2}\right)\right\}, \\
\mathcal{N}_{l n ; \text { scalar }}^{\left(\eta_{2}\right)}(\vec{k})=-8 \mathcal{A}_{l n}\left(k_{\perp}^{2}\right) k_{\perp}^{2} k_{z}^{2}, \\
\mathcal{N}_{l n ; \text { scalar }}^{\left(\eta_{3}\right)}(\vec{k})=\mathcal{N}_{l n ; \text { scalar }}^{\left(\eta_{4}\right)}(\vec{k})=0, \\
\mathcal{N}_{l n ; \text { scalar }}^{\left(\zeta_{\perp}\right)}(\vec{k})=\frac{4}{3} \mathcal{A}_{l n}\left(k_{\perp}^{2}\right)\left\{(1-\theta-\phi) \omega_{k l}^{2}+(\theta+\phi) k_{\perp}^{2}-(1+\theta+\phi)\left(k_{z}^{2}+m^{2}\right)\right\} \\
\times\left\{(3-3 \theta-2 \phi) \omega_{k l}^{2}+(1+3 \theta+2 \phi) k_{\perp}^{2}-(1+3 \theta+2 \phi) k_{z}^{2}-(3+3 \theta+2 \phi) m^{2}\right\}, \\
\mathcal{N}_{l n ; \text { scalar }}^{\left(\zeta_{l l}\right)}(\vec{k})=\frac{4}{3} \mathcal{A}_{l n}\left(k_{\perp}^{2}\right)\left\{(1-\theta) \omega_{k l}^{2}+(1+\theta) k_{\perp}^{2}+(1-\theta) k_{z}^{2}-(1+\theta) m^{2}\right\} \\
\times\left\{(3-3 \theta-2 \phi) \omega_{k l}^{2}+(1+3 \theta+2 \phi) k_{\perp}^{2}-(1+3 \theta+2 \phi) k_{z}^{2}-(3+3 \theta+2 \phi) m^{2}\right\}, \\
\mathcal{N}_{l n ; \text { Dirac }}^{\left(\eta_{\eta}\right)}(\vec{k})=2 \mathcal{D}_{l n}\left(k_{\perp}^{2}\right) k_{\perp}^{2}\left(-\omega_{k l}^{2}+k_{z}^{2}+m^{2}\right),
\end{gathered}
$$




$$
\begin{aligned}
& \mathcal{N}_{l n ; \text { Dirac }}^{\left(\eta_{1}\right)}(\vec{k})=8\left[8 \mathcal{B}_{l n}\left(k_{\perp}^{2}\right) k_{\perp}^{2}\left\{-2 \omega_{k l}^{2}+(1+\theta)(1+2 \theta+2 \phi) k_{\perp}^{2}+2 \theta(1+\theta+\phi) k_{z}^{2}+2(1+\theta)(1+\theta+\phi) m^{2}\right\}\right. \\
& +2 \mathcal{C}_{l n}\left(k_{\perp}^{2}\right)\left\{\omega_{k l}^{4}+\theta(1+\theta+\phi) k_{z}^{4}+(1+\theta)(1+\theta+\phi) m^{4}-\left(1+3 \theta+2 \phi-\theta \phi-\theta^{2}\right) \omega_{k l}^{2} k_{z}^{2}\right. \\
& \left.+(1+2 \theta)(1+\theta+\phi) k_{z}^{2} m^{2}-\left(2+2 \theta+\phi-\theta \phi-\theta^{2}\right) \omega_{k l}^{2} m^{2}\right\} \\
& +\mathcal{D}_{l n}\left(k_{\perp}^{2}\right) k_{\perp}^{2}(1+\theta)(1+2 \theta+2 \phi)\left(\omega_{k l}^{2}-k_{z}^{2}-m^{2}\right) \\
& \left.+2 \mathcal{E}_{l n}\left(k_{\perp}^{2}\right) k_{\perp}^{2}\left\{(3+4 \theta+2 \phi) \omega_{k l}^{2}-k_{z}^{2}\left(2+5 \theta+2 \phi+4 \theta \phi+4 \theta^{2}\right)-(1+\theta)(3+4 \theta+4 \phi) m^{2}\right\}\right], \\
& \mathcal{N}_{l n ; \text { Dirac }}^{\left(\eta_{2}\right)}(\vec{k})=8 \mathcal{B}_{l n}\left(k_{\perp}^{2}\right) k_{\perp}^{4}+\mathcal{C}_{l n}\left(k_{\perp}^{2}\right) k_{\perp}^{2}\left(\omega_{k l}^{2}+k_{z}^{2}-m^{2}\right)+2 \mathcal{D}_{l n}\left(k_{\perp}^{2}\right) k_{z}^{2}\left(\omega_{k l}^{2}-k_{z}^{2}-m^{2}\right)+4 \mathcal{E}_{l n}\left(k_{\perp}^{2}\right) k_{\perp}^{2} k_{z}^{2}, \\
& \mathcal{N}_{l n ; \text { Dirac }}^{\left(\eta_{3}\right)}(\vec{k})=\mathcal{N}_{l n ; \text { Dirac }}^{\left(\eta_{4}\right)}(\vec{k})=0 \\
& \mathcal{N}_{l n ; \text { Dirac }}^{\left(\zeta_{\perp}\right)}(\vec{k})=\frac{4}{3}\left[8 \mathcal { B } _ { l n } ( k _ { \perp } ^ { 2 } ) k _ { \perp } ^ { 2 } \left\{6 \omega_{k l}^{2}-(1+2 \theta+2 \phi)(2+3 \theta+2 \phi) k_{\perp}^{2}-2(1+\theta+\phi)(2+3 \theta+2 \phi) k_{z}^{2}\right.\right. \\
& \left.-2(1+\theta+\phi+1)(3+3 \theta+2 \phi) m^{2}\right\}+2 \mathcal{C}_{l n}\left(k_{\perp}^{2}\right)\left\{-3 \omega_{k l}^{4}-(1+\theta+\phi)(2+3 \theta+2 \phi) k_{z}^{4}\right. \\
& -(1+\theta+\phi)(3+3 \theta+2 \phi) m^{4}+\left(5+7 \theta+6 \phi-3 \theta^{2}-5 \theta \phi-2 \phi^{2}\right) \omega_{k l}^{2} k_{z}^{2}-(1+\theta+\phi)(5+6 \theta+4 \phi) k_{z}^{2} m^{2} \\
& \left.+\left(6+6 \theta+5 \phi-3 \theta^{2}-5 \theta \phi-2 \phi^{2}\right) \omega_{k l}^{2} m^{2}\right\}+\mathcal{D}_{l n}\left(k_{\perp}^{2}\right) k_{\perp}^{2}(1+2 \theta+2 \phi)(2+3 \theta+2 \phi)\left(-\omega_{k l}^{2}+k_{z}^{2}+m^{2}\right) \\
& +2 \mathcal{E}_{l n}\left(k_{\perp}^{2}\right) k_{\perp}^{2}\left\{-\omega_{k l}^{2}(7+12 \theta+10 \phi)+(2+3 \theta+2 \phi)(3+4 \theta+4 \phi) k_{z}^{2}\right. \\
& \left.\left.+\left(7+19 \theta+16 \phi+12 \theta^{2}+20 \theta \phi+8 \phi^{2}\right) m^{2}\right\}\right], \\
& \mathcal{N}_{l n ; \text { Dirac }}^{\left(\zeta_{\|}\right)}(\vec{k})=\frac{8}{3}\left[8 \mathcal{B}_{l n}\left(k_{\perp}^{2}\right) k_{\perp}^{2}\left\{3 \omega_{k l}^{2}-(1+\theta)(2+3 \theta+2 \phi) k_{\perp}^{2}-\theta(2+3 \theta+2 \phi) k_{z}^{2}-(1+\theta)(3+3 \theta+2 \phi) m^{2}\right\}\right. \\
& -\mathcal{C}_{l n}\left(k_{\perp}^{2}\right)\left\{3 \omega_{k l}^{4}+\theta(2+3 \theta+2 \phi) k_{z}^{4}+(1+\theta)(3+3 \theta+2 \phi) m^{4}-\omega_{k l}^{2} k_{z}^{2}\left(1+10 \theta+4 \phi-3 \theta^{2}-2 \theta \phi\right)\right. \\
& \left.+\left(1+8 \theta+2 \phi+6 \theta^{2}+4 \theta \phi\right) k_{z}^{2} m^{2}-\left(6+6 \theta+2 \phi-3 \theta^{2}-2 \theta \phi\right) \omega_{k l}^{2} m^{2}\right\} \\
& +\mathcal{D}_{l n}\left(k_{\perp}^{2}\right) k_{\perp}^{2}(1+\theta)(2+3 \theta+2 \phi)\left(-\omega_{k l}^{2}+k_{z}^{2}+m^{2}\right) \\
& \left.+2 \mathcal{E}_{l n}\left(k_{\perp}^{2}\right) k_{\perp}^{2}\left\{-\omega_{k l}^{2}(5+6 \theta+2 \phi)+(1+2 \theta)(2+3 \theta+2 \phi) k_{z}^{2}+(1+\theta)(5+6 \theta+4 \phi) m^{2}\right\}\right] .
\end{aligned}
$$

During our entire calculation, $\Gamma$ is introduced as a parameter, although it can be calculated microscopically from the interaction Lagrangian of a particular system and one can get it as temperature $(T)$, magnetic field $(B)$ and momentum $\vec{k}$-dependent function. By taking the appropriate momentum average one can get momentum-independent $\Gamma$ and take outside the $d^{2} k_{\perp}$ integral of Eq. (50). So, considering $\Gamma$ as constant or independent of $k_{\perp}$, the $d^{2} k_{\perp}$ integral of Eq. (50) can be analytically performed and we get the following simplified expressions of the viscous coefficients in the presence of a constant external magnetic field:

$v=\xi^{(v)} \eta_{0}+\sum_{l=0}^{\infty} \sum_{n=0}^{\infty} \frac{1}{T} \int_{-\infty}^{\infty} \frac{d k_{z}}{(2 \pi)} \frac{1}{4 \omega_{k l} \omega_{k n}} \frac{\Gamma}{\left(\omega_{k l}-\omega_{k n}\right)^{2}+\Gamma^{2}}\left\{a f_{a}\left(\omega_{k l}\right)+a f_{a}\left(\omega_{k n}\right)+2 f_{a}\left(\omega_{k l}\right) f_{a}\left(\omega_{k n}\right)\right\} \tilde{\mathcal{N}}_{l n}^{(v)}\left(k_{z}\right)$,

where

$$
\tilde{\mathcal{N}}_{l n}^{(v)}\left(k_{z}\right)=\int \frac{d^{2} k_{\perp}}{(2 \pi)^{2}} \mathcal{N}_{l n}^{(v)}(\vec{k})
$$

Substituting Eqs. (52)-(63) into Eq. (65), we obtain

$$
\tilde{\mathcal{N}}_{l n ; \text { scalar }}^{\left(\eta_{0}\right)}(\vec{k})=2 \mathcal{A}_{l n}^{(4)},
$$




$$
\begin{aligned}
\tilde{\mathcal{N}}_{l n \text {;scalar }}^{\left(\eta_{1}\right)}(\vec{k})= & 8\left[\mathcal{A}_{l n}^{(0)}\left\{(1-\theta)\left(\omega_{k l}^{2}+k_{z}^{2}\right)-(1+\theta) m^{2}\right\}\left\{-(1-\theta-\phi) \omega_{k l}^{2}+(1+\theta+\phi)\left(k_{z}^{2}+m^{2}\right)\right\}\right. \\
& +\mathcal{A}_{l n}^{(2)}\left\{-\left(1+\theta-2 \theta^{2}-2 \theta \phi\right) \omega_{k l}^{2}\left(1+\theta+2 \theta^{2}+2 \theta \phi\right) k_{z}^{2}+(1+\theta+1)(1+2 \theta+2 \phi) m^{2}\right\} \\
& \left.-\mathcal{A}_{l n}^{(4)}(1+\theta)(\theta+\phi)\right],
\end{aligned}
$$

$$
\begin{gathered}
\tilde{\mathcal{N}}_{l n ; \text { scalar }}^{\left(\eta_{2}\right)}(\vec{k})=-8 \mathcal{A}_{l n}^{(2)} k_{z}^{2}, \\
\tilde{\mathcal{N}}_{l n ; \text { scalar }}^{\left(\eta_{3}\right)}(\vec{k})=\tilde{\mathcal{N}}_{l n ; \text { scalar }}^{\left(\eta_{4}\right)}(\vec{k})=0,
\end{gathered}
$$

$$
\begin{aligned}
\tilde{\mathcal{N}}_{l n ; \text { scalar }}^{\left(\zeta_{\perp}\right)}(\vec{k})= & \frac{1}{3}\left[4 \mathcal{A}_{l n}^{(0)}\left\{(1-\theta-\phi) \omega_{k l}^{2}-(1+\theta+\phi)\left(k_{z}^{2}+m^{2}\right)\right\}\left\{(3-3 \theta-2 \phi) \omega_{k l}^{2}-(1+3 \theta+2 \phi) k_{z}^{2}-(3+3 \theta+2 \phi) m^{2}\right\}\right. \\
& +\mathcal{A}_{l n}^{(2)}\left\{4\left(1+10 \theta+4 \phi-6 \theta^{2}-10 \theta \phi-4 \phi^{2}\right) \omega_{k l}^{2}-4(1+2 \theta+2 \phi)(1+3 \theta+2 \phi) k_{z}^{2}\right. \\
& \left.\left.-4\left(1+7 \theta+6 \phi+6 \theta^{2}+10 \theta \phi+4 \phi^{2}\right) m^{2}\right\}+4 \mathcal{A}_{l n}^{(4)}(\theta+\phi)(1+3 \theta+2 \phi)\right],
\end{aligned}
$$

$$
\begin{aligned}
\tilde{\mathcal{N}}_{l n ; \text {;calar }}^{(\zeta)}(\vec{k})= & \frac{4}{3}\left[\mathcal{A}_{l n}^{(0)}\left\{(1-\theta)\left(\omega_{k l}^{2}+k_{z}^{2}\right)-(1+\theta 1) m^{2}\right\}\left\{(3-3 \theta-2 \phi) \omega_{k l}^{2}-(1+3 \theta+2 \phi) k_{z}^{2}-(3+3 \theta+2 \phi) m^{2}\right\}\right. \\
& +2 \mathcal{A}_{l n}^{(2)}\left\{\left(2+\theta-3 \theta^{2}-2 \theta \phi\right) \omega_{k l}^{2}-\theta(1+3 \theta+2 \phi) k_{z}^{2}-(1+\theta)(2+3 \theta+2 \phi) m^{2}\right\} \\
& \left.+\mathcal{A}_{l n}^{(4)}(1+\theta)(1+3 \theta+2 \phi)\right],
\end{aligned}
$$

$$
\tilde{\mathcal{N}}_{l n ; \text { Dirac }}^{\left(\eta_{0}\right)}(\vec{k})=-2 \mathcal{D}_{l n}^{(2)}\left(\omega_{k l}^{2}-k_{z}^{2}-m^{2}\right),
$$

$$
\begin{aligned}
& \tilde{\mathcal{N}}_{l n ; \text { Dirac }}^{\left(\eta_{1}\right)}(\vec{k})= 8\left[16 \mathcal{B}_{l n}^{(2)}\left\{-\omega_{k l}^{2}+\theta(1+\theta+\phi) k_{z}^{2}+(1+\theta)(1+\theta+\phi) m^{2}\right\}+8 \mathcal{B}_{l n}^{(4)}(1+\theta)(1+2 \theta+2 \phi)\right. \\
&+2 \mathcal{C}_{l n}^{(0)}\left\{\omega_{k l}^{4}+\theta(1+\theta+\phi) k_{z}^{4}+(1+\theta)(1+\theta+\phi) m^{4}-\left(1+3 \theta+2 \phi-\theta^{2}-\theta \phi\right) \omega_{k l}^{2} k_{z}^{2}\right. \\
&\left.+(1+2 \theta)(1+\theta+\phi) k_{z}^{2} m^{2}-\left(2+2 \theta+\phi-\theta^{2}-\theta \phi\right) \omega_{k l}^{2} m^{2}\right\}+\mathcal{D}_{l n}^{(2)}(1+\theta)(1+2 \theta+2 \phi)\left(\omega_{k l}^{2}-k_{z}^{2}-m^{2}\right) \\
&\left.+2 \mathcal{E}_{l n}^{(2)}\left\{(3+4 \theta+2 \phi) \omega_{k l}^{2}-\left(2+5 \theta+2 \phi+4 \theta^{2}+4 \theta \phi\right) k_{z}^{2}-(1+\theta)(3+4 \theta+4 \phi) m^{2}\right\}\right],(73) \\
& \tilde{\mathcal{N}}_{l n ; \text { Dirac }}^{\left(\eta_{2}\right)}(\vec{k})=8 \mathcal{B}_{l n}^{(4)}+\mathcal{C}_{l n}^{(2)}\left(\omega_{k l}^{2}+k_{z}^{2}-m^{2}\right)+2 \mathcal{D}_{l n}^{(0)} k_{z}^{2}\left(\omega_{k l}^{2}-k_{z}^{2}-m^{2}\right)+4 \mathcal{E}_{l n}^{(2)} k_{z}^{2}, \\
& \tilde{\mathcal{N}}_{l n ; \text { Dirac }}^{\left(\eta_{3}\right)}(\vec{k})=\tilde{\mathcal{N}}_{l n ; \text { Dirac }}^{\left(\eta_{4}\right)}(\vec{k})=0,
\end{aligned}
$$

$$
\begin{aligned}
\tilde{\mathcal{N}}_{l n ; \text { Dirac }}^{\left(\zeta_{\perp}\right)}(\vec{k})= & \frac{4}{3}\left[16 \mathcal{B}_{l n}^{(2)}\left\{3 \omega_{k l}^{2}-\left(2+5 \theta+4 \phi+3 \theta^{2}+5 \theta \phi+2 \phi^{2}\right) k_{z}^{2}-\left(3+6 \theta+5 \phi+3 \theta^{2}+5 \theta \phi+2 \phi^{2}\right) m^{2}\right\}\right. \\
& -8 \mathcal{B}_{l n}^{(4)}\left(2+7 \theta+6 \phi+6 \theta^{2}+10 \theta \phi+4 \phi^{2}\right)-2 \mathcal{C}_{l n}^{(0)}\left\{3 \omega_{k l}^{4}+\left(2+5 \theta+4 \phi+3 \theta^{2}+5 \theta \phi+2 \phi^{2}\right) k_{z}^{4}\right. \\
& +\left(3+6 \theta+5 \phi+3 \theta^{2}+5 \theta \phi+2 \phi^{2}\right) m^{4}-\left(5+7 \theta+6 \phi-3 \theta^{2}-5 \theta \phi-2 \phi^{2}\right) \omega_{k l}^{2} k_{z}^{2} \\
& \left.+\left(5+11 \theta+9 \phi+6 \theta^{2}+10 \theta \phi+4 \phi^{2}\right) k_{z}^{2} m^{2}-\left(6+6 \theta+5 \phi-3 \theta^{2}-5 \theta \phi-2 \phi^{2}\right) \omega_{k l}^{2} m^{2}\right\} \\
& -\mathcal{D}_{l n}^{(2)}\left(2+7 \theta+6 \phi+6 \theta^{2}+10 \theta \phi+4 \phi^{2}\right)\left(\omega_{k l}^{2}-k_{z}^{2}-m^{2}\right)-2 \mathcal{E}_{l n}^{(2)}\left\{\omega_{k l}^{2}(7+12 \theta+10 \phi)\right. \\
& \left.\left.-\left(6+17 \theta+14 \phi+12 \theta^{2}+20 \theta \phi+8 \phi^{2}\right) k_{z}^{2}-m^{2}\left(7+19 \theta+16 \phi+12 \theta^{2}+20 \theta \phi+8 \phi^{2}\right)\right\}\right],
\end{aligned}
$$

$$
\begin{aligned}
\tilde{\mathcal{N}}_{l n ; \text { Dirac }}^{\left(\zeta_{\|}\right)}(\vec{k})= & \frac{8}{3}\left[8 \mathcal{B}_{l n}^{(2)}\left\{3 \omega_{k l}^{2}-\theta(2+3 \theta+2 \phi) k_{z}^{2}-(1+\theta)(3+3 \theta+2 \phi) m^{2}\right\}-8 \mathcal{B}_{l n}^{(4)}(1+\theta)(2+3 \theta+2 \phi)\right. \\
& -\mathcal{C}_{l n}^{(0)}\left\{3 \omega_{k l}^{4}+\theta(2+3 \theta+2 \phi) k_{z}^{4}+(1+\theta)(3+3 \theta+2 \phi) m^{4}-\left(1+10 \theta+4 \phi-3 \theta^{2}-2 \theta \phi\right) \omega_{k l}^{2} k_{z}^{2}\right. \\
& \left.+\left(1+8 \theta+2 \phi+6 \theta^{2}+4 \theta \phi\right) k_{z}^{2} m^{2}-\left(6+6 \theta+2 \phi-3 \theta^{2}-2 \theta \phi\right) \omega_{k l}^{2} m^{2}\right\}-\mathcal{D}_{l n}^{(2)}(1+\theta)(2+3 \theta+2 \phi) \\
& \left.\times\left(\omega_{k l}^{2}-k_{z}^{2}-m^{2}\right)-2 \mathcal{E}_{l n}^{(2)}\left\{(5+6 \theta+2 \phi) \omega_{k l}^{2}-(1+2 \theta)(2+3 \theta+2 \phi) k_{z}^{2}-(1+\theta)(5+6 \theta+4 \phi) m^{2}\right\}\right],
\end{aligned}
$$

where 


$$
\begin{aligned}
& \mathcal{A}_{\text {ln }}^{(j)}=\int \frac{d^{2} k_{\perp}}{(2 \pi)^{2}} \mathcal{A}_{l n}\left(k_{\perp}^{2}\right)\left(k_{\perp}^{2}\right)^{j / 2}, \\
& \mathcal{B}_{\text {ln }}^{(j)}=\int \frac{d^{2} k_{\perp}}{(2 \pi)^{2}} \mathcal{B}_{l n}\left(k_{\perp}^{2}\right)\left(k_{\perp}^{2}\right)^{j / 2}, \\
& \mathcal{C}_{l n}^{(j)}=\int \frac{d^{2} k_{\perp}}{(2 \pi)^{2}} \mathcal{C}_{l n}\left(k_{\perp}^{2}\right)\left(k_{\perp}^{2}\right)^{j / 2}, \\
& \mathcal{D}_{l n}^{(j)}=\int \frac{d^{2} k_{\perp}}{(2 \pi)^{2}} \mathcal{D}_{l n}\left(k_{\perp}^{2}\right)\left(k_{\perp}^{2}\right)^{j / 2}, \\
& \mathcal{E}_{l n}^{(j)}=\int \frac{d^{2} k_{\perp}}{(2 \pi)^{2}} \mathcal{E}_{l n}\left(k_{\perp}^{2}\right)\left(k_{\perp}^{2}\right)^{j / 2} .
\end{aligned}
$$

Exploiting the orthogonality of the Laguerre polynomials present in the functions $\mathcal{A}_{l n}\left(k_{\perp}^{2}\right), \mathcal{B}_{l n}\left(k_{\perp}^{2}\right), \ldots, \mathcal{E}_{l n}\left(k_{\perp}^{2}\right)$, the $d^{2} k_{\perp}$ integrals of Eqs. (78)-(82) are now performed and the analytic expressions of the quantities $\mathcal{A}_{l n}^{(j)}, \mathcal{B}_{l n}^{(j)}, \ldots, \mathcal{E}_{l n}^{(j)}$ are listed in Appendix C.

\section{NUMERICAL RESULTS AND DISCUSSIONS}

In this section, we will try to explore the numerical outcomes of shear and bulk viscosity components; mainly, their temperature and magnetic-field-dependent curves will be our matter of interest. To show numerical results of the viscosities, we have chosen systems consisting of massless particles for simplicity, although the final expressions for the viscous coefficients in Eqs. (64)-(77) are valid for massive particles as well. We have also performed a proper numerical consistency check for ensuring the correctness of the analytical expressions as well as the numerical codes as follows: we have taken the numerical limit $B \rightarrow 0$ of the viscous coefficients in the presence of a magnetic field [Eq. (64)] and found that, for sufficiently small values of $B$, $\eta_{0} \rightarrow \eta, \eta_{1} \rightarrow 0, \eta_{2} \rightarrow 0, \zeta_{\perp} \rightarrow \zeta$ and $\zeta_{\|} \rightarrow \zeta$, where $\eta$ and $\zeta$ are, respectively, the shear and bulk viscosities in the absence of the external magnetic field obtained from Eqs. (38)-(41). This consistency implies that, for sufficiently small values of $B$, a large number of Landau levels contribute to $v$ in Eq. (64) (the Landau levels become infinitesimally close to each other reaching the continuum), which in turn numerically reproduce the exact continuum results of Eqs. (38)-(41), though it is nontrivial to take an analytic $B \rightarrow 0$ limit of Eq. (64). For all the numerical results shown in this section, we have considered up to 10000 Landau levels.

Figure 2 shows the temperature and magnetic field dependence of $\eta_{0}$ for the two different systems consisting of spin-0 scalar bosons and spin- $\frac{1}{2}$ Dirac fermions. Figures 2(a) and 2(c) depict the variation of the dimensionless quantity $\eta_{0} / T^{3}$ as a function of temperature for different values of magnetic field whereas Figs. 2(b) and 2(d) show the variation of $\eta_{0} / T^{3}$ as a function of magnetic field for different values of temperature. To understand the change in the values of viscosity components due to the magnetic field, we have first estimated viscosities at $B=0$. Using Eqs. (38) and (39), the shear viscosity $\eta$ of scalar and Dirac fluids at $B=0$ are estimated and they are plotted in Fig. 2 with solid red lines. With respect to the $B=0$ curves, we can get a comparative measurement on the values of the $\eta_{0}$ component. For the scalar fluid, $\eta_{0}$ decreases with $B$ and increases with $T$. One can identify the opposite roles of $T$ and $B$ on transport coefficients, which is also noticed in RTA of kinetic theory approaches $[10,14]$. Physically, we can also comprehend that temperature is the measurement of randomness, while the magnetic field aligns the system. So their thermodynamical roles on the system are expected to be quite opposite in nature.

A detail quantitative understanding for $T$ and $B$ dependence of $\eta_{0}$ seems to be a very cumbersome task (as the analytical expressions are very complicated), but we can try to relate it with its RTA expression $[10,14]$

$$
\eta_{0}=\eta A_{0} \quad \text { with } \quad A_{0}=\frac{1}{1+4\left(\frac{\tau_{c}}{\tau_{B}}\right)^{2}},
$$

where the expression of $\eta$ will be same as given in Eqs. (38) and (39), $\tau_{c}=1 / \Gamma$ is basically the relaxation time or inverse of the thermal width, and $\tau_{B}=\frac{\omega_{\mathrm{av}}}{e B}$ is the inverse of synchrotron frequency. Now in the quantum mechanical picture, the energy difference between the two Landau levels $\Delta \omega=\left(\omega_{k l}-\omega_{k n}\right)$ might be associated to the synchrotron frequency; i.e., we can grossly write $\Delta \omega \approx 1 / \tau_{B}$. Using this connection in Eq. (64), one can identify the term of effective relaxation time:

$$
\frac{\Gamma}{\left(\omega_{k l}-\omega_{k n}\right)^{2}+\Gamma^{2}} \approx \tau_{c} \frac{1}{1+\left(\frac{\tau_{c}}{\tau_{B}}\right)^{2}} \approx \tau_{c} A_{0} .
$$

In the massless limit, $\tau_{B} \approx \frac{3 T}{e B}[10]$, so the dominant $B$-dependent anisotropic factor

$$
A_{0}(T, B) \approx\left[1+\left(\frac{2 \tau_{c} e B}{3 T}\right)^{2}\right]^{-1}
$$

will mainly control the $T$ and $B$ dependence of $\eta_{0}$. One can find that $A_{0}$ decreases with $B$ and increases with $T$, which is mostly reflected on $\eta_{0}(T, B)$. Apart from the anisotropic factor $A_{0}, \eta_{0}$ contains the additional $B$ dependence via the quantized energy relation in the other part of the integrand of Eq. (64). The other part of the integrand mainly contains $\tilde{N}_{l n}(\omega)$ as well as the thermal distribution function $f(\omega)$, which is not much influential for scalar fluid. Therefore, $B$ and $T$ dependence of $\eta_{0}$ almost follows the same trend as observed in $A_{0}(T, B)$. 

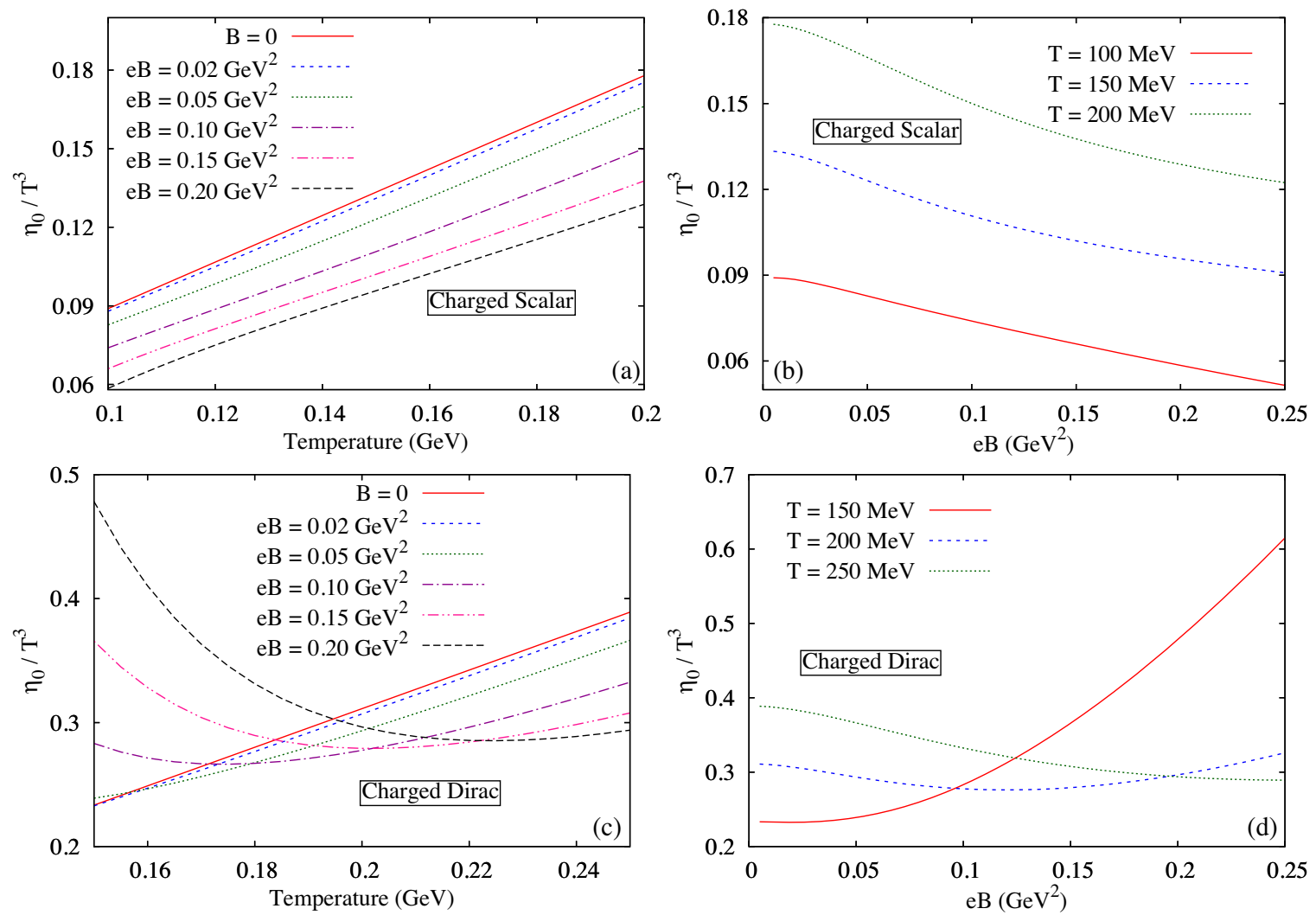

FIG. 2. The variation of $\eta_{0} / T^{3}$ as a function of (a) $T$ and (b) $e B$ for system of massless charged scalar bosons (spin 0 ) with relaxation time $\tau_{c}=1 / \Gamma=1 \mathrm{fm}$. The variation of $\eta_{0} / T^{3}$ as a function of (c) $T$ and (d) $e B$ for system of massless charged Dirac fermions (spin $\frac{1}{2}$ ) with relaxation time $\tau_{c}=1 / \Gamma=1 \mathrm{fm}$.

For the Dirac fluid, a similar trend for $\eta_{0}$ is observed at the lower values of magnetic field and high-temperature regions. However, we notice nonmonotonic behavior of $\eta_{0}$ at higher values of the external magnetic field. This is probably due to the nontrivial spin structure contained in $\tilde{N}_{l n}(\omega)$ for the Dirac case and is mainly responsible for this nonmonotonic behavior.

Since the $\tilde{N}_{l n}$ 's corresponding to the other viscous coefficients also carry the magnetothermodynamical quantities $\theta=\left(\frac{\partial P}{\partial \varepsilon}\right)_{B}$ and $\phi=-B\left(\frac{\partial M}{\partial \varepsilon}\right)_{B}$, their temperature and magnetic field dependence are separately plotted in Figs. 3 and 4. Their detail calculations are provided in Appendix D. Figures 3(a) and 2(c) depict the variation of the $\theta$ as a function of temperature whereas Figs. 3(b) and 3(d) show the variation of $\theta$ as a function of magnetic field.

We first note that, at $B=0, \theta$ is nothing but the squared speed of sound $\left(c_{s}^{2}\right)$ of the medium, which is $\frac{1}{3}$ in the massless case as clearly shown in Figs. 3(a) and 3(c) by solid red horizontal lines. However, at $B \neq 0$, the quantity $\theta=\left(\frac{\partial P}{\partial \varepsilon}\right)_{B}$ is not equal to the speed of sound of the medium since the speed of sound is most generally defined as $c_{s}^{2}=\left(\frac{\partial P}{\partial \varepsilon}\right)_{s} \neq \theta$, where $s$ is the entropy of the medium [57]. At nonzero magnetic field, we find $\theta$ to increase (decrease) monotonically with the increase in temperature for the scalar (Dirac) fluid, whereas for the scalar (Dirac) fluid, $\theta$ decreases (increases) with the increases in magnetic field. Thus, we observe completely opposite behaviors for the scalar and Dirac fluid in the $T$ and $B$ dependence of $\theta$, though, in all the cases, at high temperature, $\theta$ asymptotically approach the corresponding $B=0$ curves.

In Figs. 4(a) and 4(c), we have shown the variation of the $\phi$ as a function of temperature whereas in Figs. 4(b) and 4(d), the variation of $\phi$ as a function of magnetic field has been shown. We first note that, at vanishing magnetic field, $\phi$ becomes zero as it is related to the magnetization of the system. We also notice that $\phi$ decreases (increases) monotonically with the increase in temperature for the scalar (Dirac) fluid, whereas it increases (decreases) with the increases in magnetic field. Thus, similar to $\theta$, here also we observe completely opposite roles of temperature and magnetic field. Interestingly, we get $\phi>0$ for scalar and $\phi<0$ for Dirac systems. Like $\theta$, in all the cases, at high temperature, $\phi$ also asymptotically approach the corresponding $B=0$ curves.

Next, let us come to the corresponding temperature and magnetic field profiles for the other shear viscosity components $\eta_{1}$ and $\eta_{2}$. They are shown, respectively, in Figs. 5 and 6. Unlike $\eta_{0}$, these components are purely magnetically induced components as they are completely disappeared at 

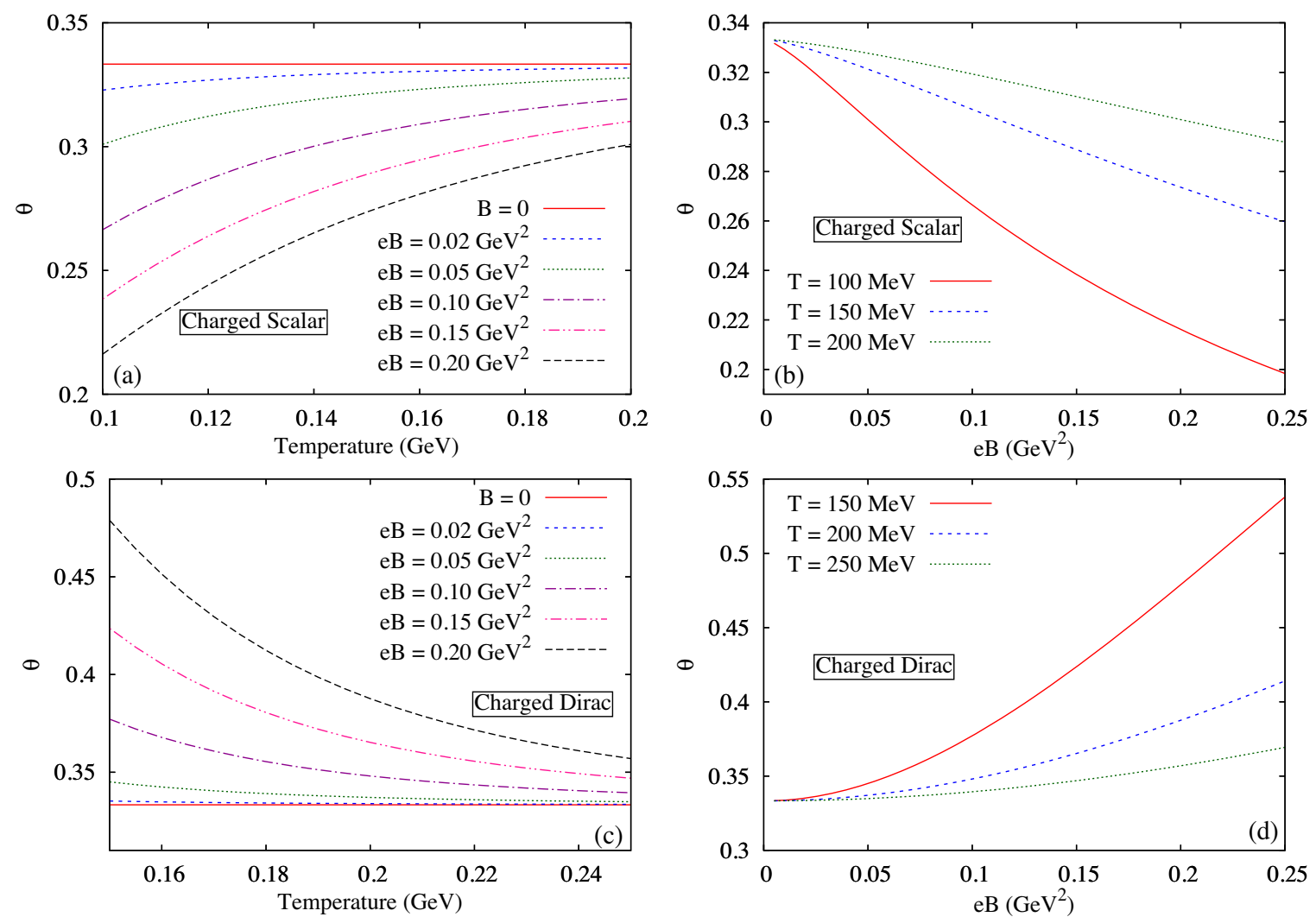

FIG. 3. The variation of $\theta=\left(\frac{\partial P}{\partial \varepsilon}\right)_{B}$ as a function of (a) $T$ and (b) $e B$ for system of massless charged scalar bosons (spin 0 ). The variation of $\theta$ as a function of (c) $T$ and (d) $e B$ for system of massless charged Dirac fermions (spin $\frac{1}{2}$ ).
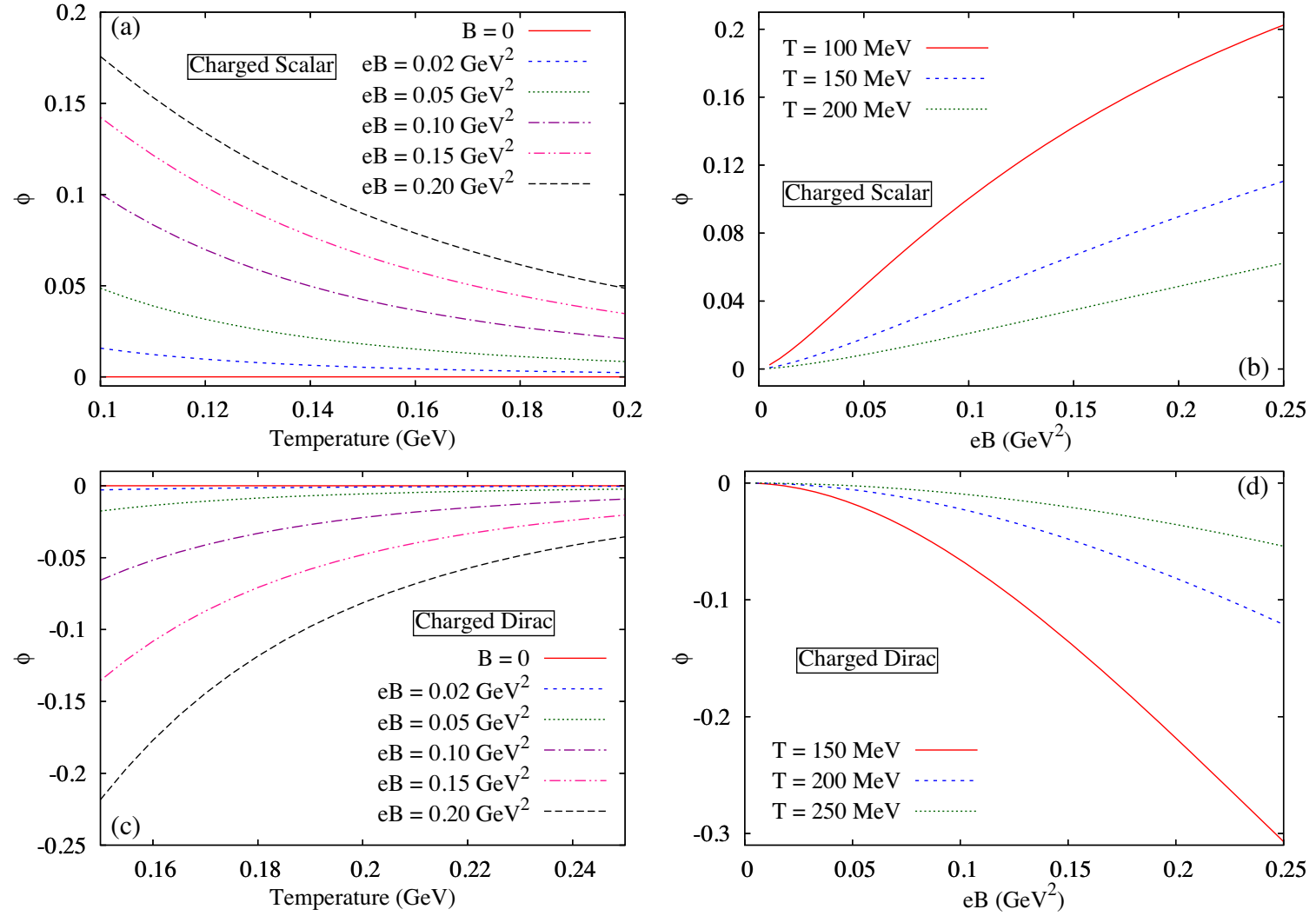

FIG. 4. The variation of $\phi=-B\left(\frac{\partial M}{\partial \varepsilon}\right)_{B}$ as a function of (a) $T$ and (b) $e B$ for system of massless charged scalar bosons (spin 0 ). The variation of $\phi$ as a function of (c) $T$ and (d) $e B$ for system of massless charged Dirac fermions (spin $\frac{1}{2}$ ). 

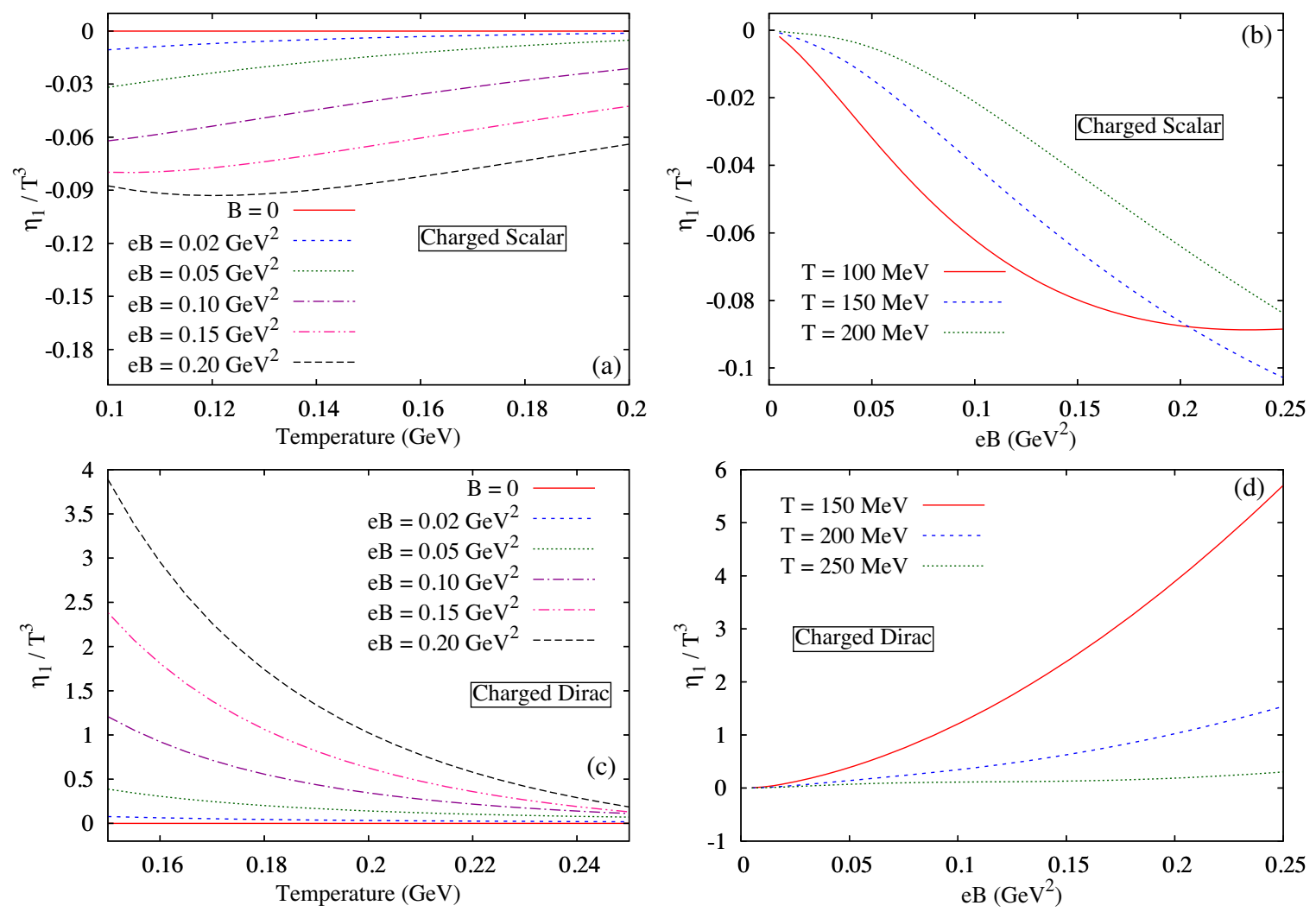

FIG. 5. The variation of $\eta_{1} / T^{3}$ as a function of (a) $T$ and (b) $e B$ for system of massless charged scalar bosons (spin 0 ) with relaxation time $\tau_{c}=1 / \Gamma=1 \mathrm{fm}$. The variation of $\eta_{1} / T^{3}$ as a function of (c) $T$ and (d) $e B$ for system of massless charged Dirac fermions (spin $\frac{1}{2}$ ) with relaxation time $\tau_{c}=1 / \Gamma=1 \mathrm{fm}$.

$B=0$, while $\eta_{0}$ at $B=0$ becomes exactly equal to the $\eta$. In RTA of kinetic theory framework [10,14], one can find a proportional relation $\eta_{1,2} \propto\left(\tau_{c} / \tau_{B}\right)^{2}$, which is missing for $\eta_{0}$. Therefore, at $B \rightarrow 0$ or $\tau_{B} \rightarrow \infty$, one gets $\eta_{1,2} \rightarrow 0$ in RTA, whereas, in the Kubo expressions, this imposition is taken care of by the rich structure contained in $\tilde{N}_{l n}$ 's of Eq. (64) when the continuum limits $(B \rightarrow 0)$ are considered.

Figures 5(a) and 5(c) depict the variation of the dimensionless quantity $\eta_{1} / T^{3}$ as a function of temperature for different values of magnetic field whereas Figs. 5(b) and 5(d) show the variation of $\eta_{1} / T^{3}$ as a function of magnetic field for different values of temperature. For the scalar (Dirac) case, $\eta_{1} / T^{3}$ increases (decrease) monotonically with the increase in temperature and it decreases (increases) with the increase in magnetic field, though at high magnetic field region slight nonmonotonicity is observed. We also notice that $\eta_{1}$ is negative for the scalar fluid and it might not be correct to consider the absolute values of the viscosity component; rather the positive or negative sign should be considered as the direction of magnetically induced shear flow. The completely opposite behavior of the temperature and magnetic field dependence of $\eta_{1} / T^{3}$ of the scalar and Dirac fluid can be attributed to several factors; for example the different spin structures contained in $\tilde{N}_{l n}(\omega)$ 's as well as the opposite thermomagnetic behavior of $\theta$ and $\phi$. In all the cases, at high temperature, $\eta_{1}$ is seen to approach zero which is due to the increase in random thermal motions in the system trying to destroy the magnetic orientations.

Next, Figs. 6(a) and 6(c) show the variation of the dimensionless quantity $\eta_{2} / T^{3}$ as a function of temperature whereas Figs. 6(b) and 6(d) show the variation of $\eta_{2} / T^{3}$ as a function of magnetic field. We observe that $\eta_{2} / T^{3}$ increases monotonically with the increase in temperature, whereas a nonmonotonic behavior is noticed in its magnetic field dependence. Like $\eta_{1}, \eta_{2}$ is also varying from positive to negative values at different $T$ and $B$ ranges, which should again be considered as the direction of the magnetically induced shear flows.

Since the other shear viscosity components $\eta_{3}$ and $\eta_{4}$ are coming zero in quantum field theory calculations because of the antisymmetric structure, we have not plotted them. The same vanishing values are realized in the ADS/CFT direction [58,59]. However, in the RTA of kinetic-theorybased calculations $[10,14]$, one might expect their nonzero values at nonzero chemical potential of the fluid, where particle and antiparticle density becomes different. These two coefficients in kinetic theory framework are realized as the Hall viscosities, which of course are vanished at zero chemical potential, i.e., when particle and antiparticle densities are the same; but they should be finite at nonzero 

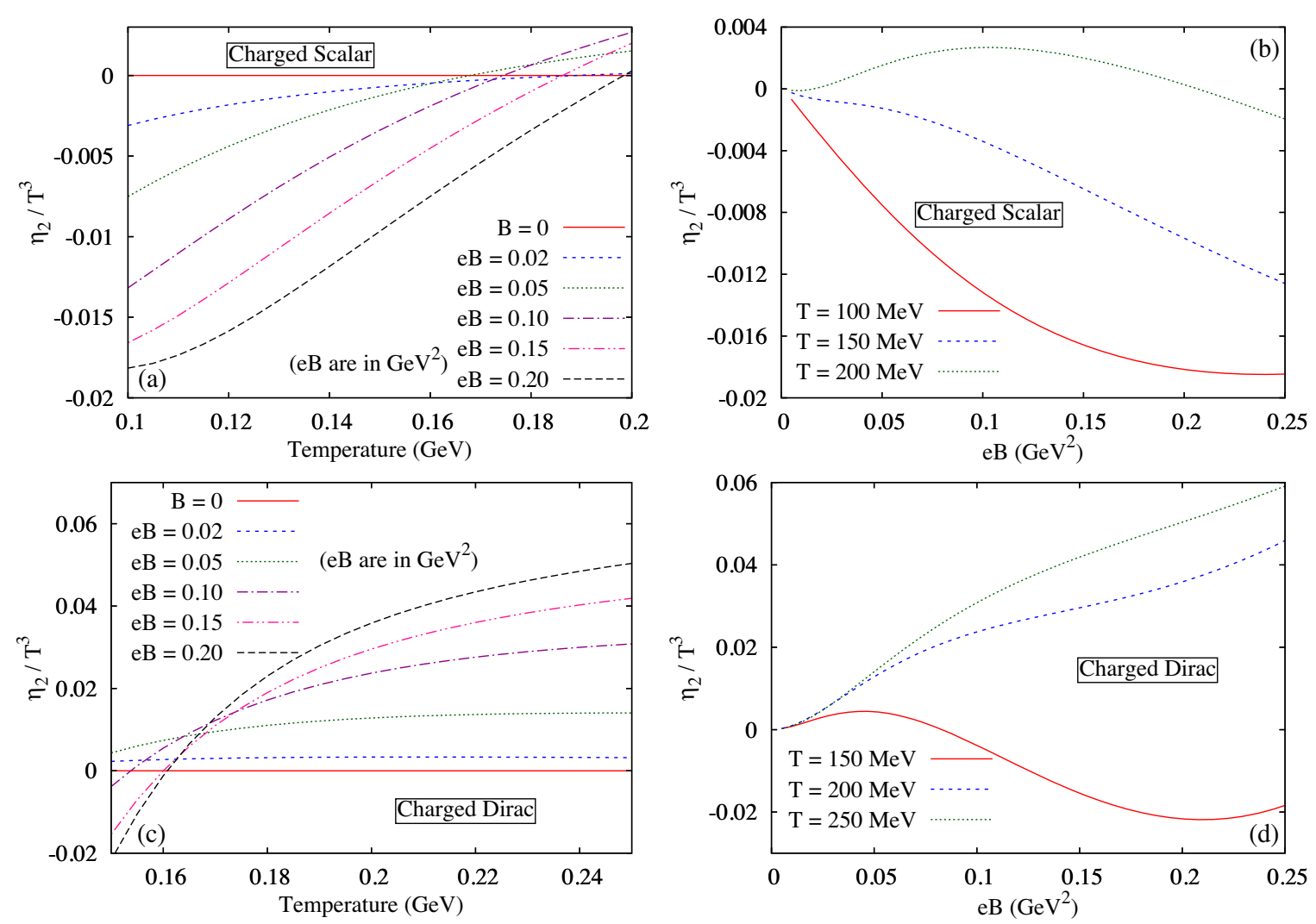

FIG. 6. The variation of $\eta_{2} / T^{3}$ as a function of (a) $T$ and (b) $e B$ for system of massless charged scalar bosons (spin 0 ) with relaxation time $\tau_{c}=1 / \Gamma=1 \mathrm{fm}$. The variation of $\eta_{2} / T^{3}$ as a function of (c) $T$ and (d) $e B$ for system of massless charged Dirac fermions (spin $\frac{1}{2}$ ) with relaxation time $\tau_{c}=1 / \Gamma=1 \mathrm{fm}$.

values of the chemical potential. For the Kubo or the field theoretical expressions, it seems that there is no possibility of getting nonzero Hall viscosity even at finite chemical potential as their vanishing contributions are coming from the antisymmetric nature of vertex factors $\tilde{N}_{l n}(\omega)$. We have not found any discussion on it in any earlier references (of course based on our searching) and we are unable to resolve this discrepancy, which should get kind attention from the community.

Now, let us turn our attention to the bulk viscosity coefficients $\zeta_{\perp}$ and $\zeta_{\|}$whose temperature and magnetic field dependence are shown in Figs. 7 and 8, respectively. Before going to finite $B$ cases, let us recapitulate our earlier knowledge of bulk viscosity at $B=0$. The expressions of $\zeta$ at $B=0$ for the scalar and Dirac system are given in Eqs. (40) and (41), respectively, from which we see that $\zeta \propto(3 \theta-1)$ in the massless limit $(m=0)$. As already discussed, in the massless limit, $\theta=\frac{1}{3}$ for $B=0$ and therefore one gets zero bulk viscosity although the shear viscosity is not necessary to be zero. At the high temperature, QCD behaves like a massless and conformal-type theory, where we can get $\zeta=0$. However, in the low and intermediate values of temperature, QCD will exhibit its nonconformal nature, which can be measured through the nonvanishing profile of $\zeta(T)$. If we take guidance from the lattice QCD calculations [60] (see references therein), then a clear deviation of $\theta$ from $\frac{1}{3}$ will be noticed and a nonzero interaction measure $T_{\mu}^{\mu}=(\varepsilon-3 P)$ of QCD thermodynamics will also be observed in the low and intermediate temperature domains. We can understand that the trace of the ideal part of the EMT is interaction measure of QCD thermodynamics; similarly the dissipation part of the EMT is basically linked with the bulk viscosity. Nonzero values of both expose the nonconformal nature of the QCD system $[61,62]$.

Here, we will not go through any particular system like QCD but rather will consider general relativistic scalar and Dirac fluids. And in the numerical point of view, we have focused on massless limits, from where we can get an idea of extreme relativistic boundaries of the different quantities. The bulk viscosity, which is zero in the massless limit of $B=0$ case, might not remain the same at finite $B$ case; one immediate reason is that $\theta$ deviates from $1 / 3$ when we switch on the magnetic field; therefore, a nonzero bulk viscosity is expected even in the massless limit. Even if one puts $\theta=\frac{1}{3}$ in Eq. (64) by hand for the massless system, the bulk viscosity components will still not be vanished due to the presence of other terms in $\tilde{N}_{l n}(\omega)$. So, it means that an additional nonconformal picture is growing at the finite magnetic field case, which is entering not only through the 

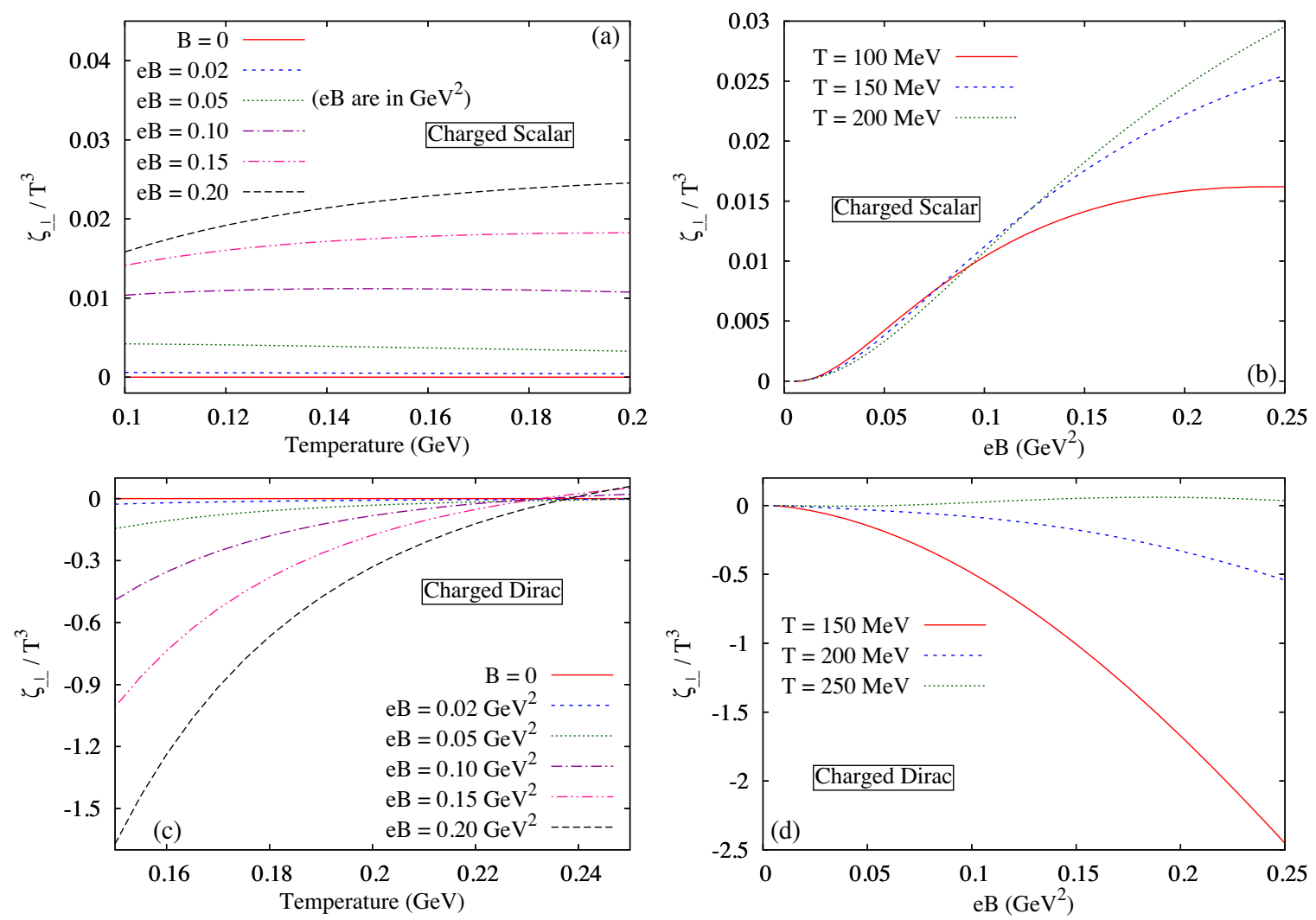

FIG. 7. The variation of $\zeta_{\perp} / T^{3}$ as a function of (a) $T$ and (b) $e B$ for system of massless charged scalar bosons (spin 0 ) with relaxation time $\tau_{c}=1 / \Gamma=1 \mathrm{fm}$. The variation of $\eta_{\perp} / T^{3}$ as a function of (c) $T$ and (d) $e B$ for system of massless charged Dirac fermions (spin $\frac{1}{2}$ ) with relaxation time $\tau_{c}=1 / \Gamma=1 \mathrm{fm}$.

$\theta(T, B)$ but also some other $B$-dependent terms in $\tilde{N}_{l n}(\omega)$. Hence, we can say that massless relativistic matter in the presence of a magnetic field can have nonzero bulk viscosity, which corresponds to its nonconformal nature (irrespective of the system like QED or QCD plasma). The phenomenon might be a little mild at high $T$ and low $B$ domain but quite prominent at low $T$ and high $B$ zone, which is known to be the quantum zone. This might be considered as a pure quantum field theoretical phenomenon due to the field quantization picture.

In Figs. 7(a) and 7(c), we depict the variation of the dimensionless quantity $\zeta_{\perp} / T^{3}$ as a function of temperature for different values of magnetic field whereas in Figs. 7(b) and 7(d), we show the variation of $\zeta_{\perp} / T^{3}$ as a function of magnetic field for different values of temperature. For the scalar fluid, $\zeta_{\perp} / T^{3}$ weakly depends on the temperature and the dependence is quite nonmonotonic. On the other hand, for the Dirac fluid, $\zeta_{\perp} / T^{3}$ increases monotonically with the increase in temperature, whereas with the increase in magnetic field, $\zeta_{\perp} / T^{3}$ increases (decreases) for the scalar (Dirac) cases; though a slight nonmonotonic behaviour is seen at the high temperature region. Like $\eta_{1}$ and $\eta_{2}$, we can see positive and negative directional shifts of the values of $\zeta_{\perp}$ from zero corresponding to the direction of magnetically induced bulk flows.
Finally, Figs. 8(a) and 8(c) depict the corresponding variation of the dimensionless quantity $\zeta_{\|} / T^{3}$ as a function of temperature whereas Figs. 8(b) and 8(d) show the variation of $\zeta_{\|} / T^{3}$ as a function of magnetic field. Like $\zeta_{\perp}$, for the scalar fluid, $\zeta_{\|} / T^{3}$ depends weakly on the temperature and the dependence is nonmonotonic in nature. For the Dirac fluid, $\zeta_{\perp} / T^{3}$ increases monotonically with the increase in temperature. With the increase in magnetic field, $\zeta_{\|} / T^{3}$ is seen to increase (decrease) for the scalar (Dirac) cases. The positive and negative directional shifts of the values of $\zeta_{\|}$from zero again correspond to the direction of magnetically induced bulk flows.

Comparing Figs. 7 and 8, one can see the difference between parallel and perpendicular components of bulk viscosity, i.e., $\zeta_{\|} \neq \zeta_{\perp}$ and the difference completely disappeared at $B=0$, where we can get the relation $\zeta_{\|}=\zeta_{\perp}=\zeta=0$. This fact reflects the transition from isotropic dissipation at $B=0$ to anisotropic dissipation at $B \neq 0$. Though a detailed analysis of differences between scalar and Dirac system for $\zeta_{\perp} / T^{3}$ and $\zeta_{\|} / T^{3}$ is not quite an easy task as their analytic expressions are quite complicated, main resources are hidden in $\tilde{N}_{l n}(\omega)$ and the thermal distribution functions. Also, the opposite thermomagnetic behaviors of $\theta$ and $\phi$ might have a role for getting different 

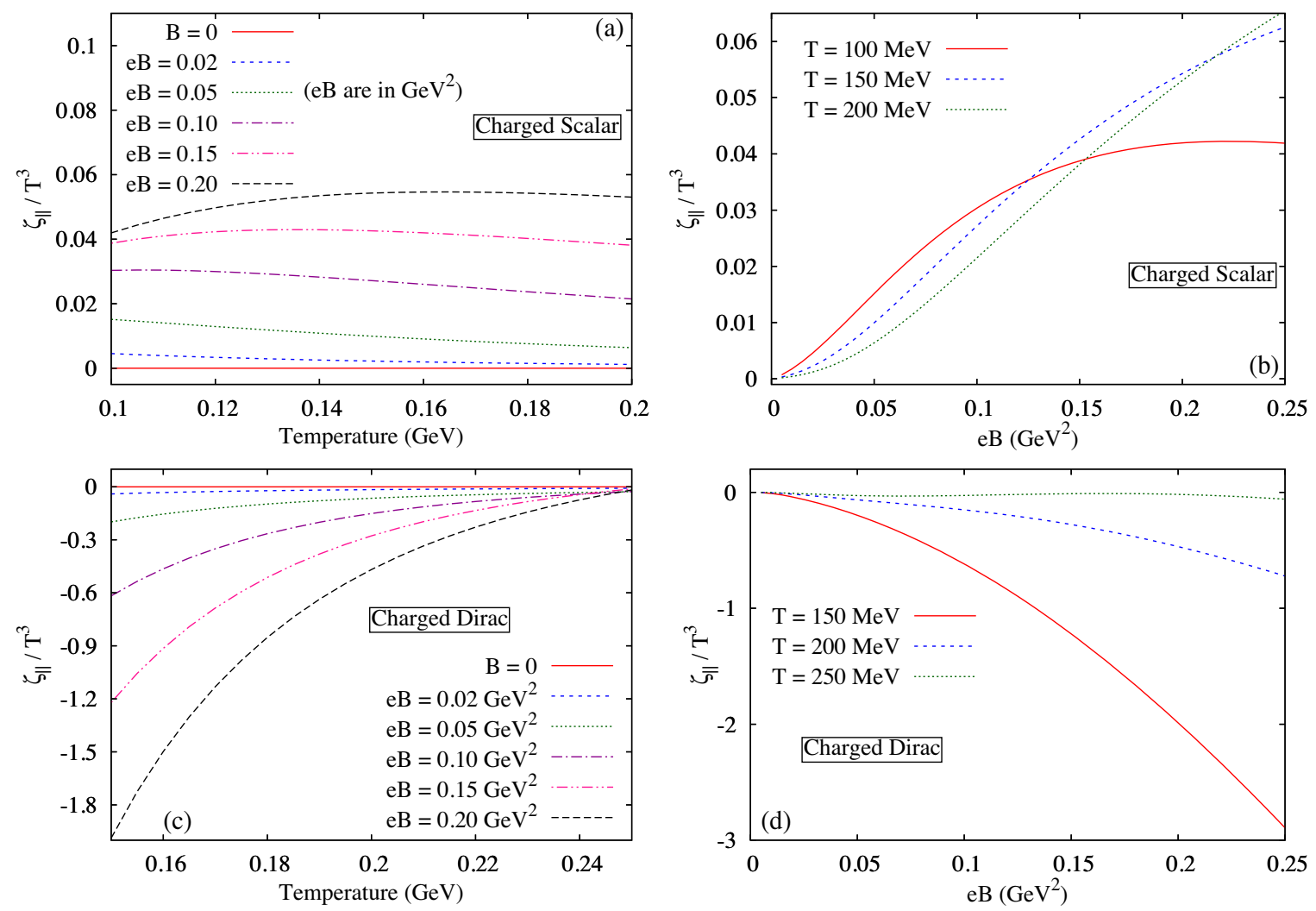

FIG. 8. The variation of $\zeta_{\|} / T^{3}$ as a function of (a) $T$ and (b) $e B$ for system of massless charged scalar bosons (spin 0 ) with relaxation time $\tau_{c}=1 / \Gamma=1 \mathrm{fm}$. The variation of $\zeta_{\|} / T^{3}$ as a function of (c) $T$ and (d) $e B$ for system of massless charged Dirac fermions (spin $\frac{1}{2}$ ) with relaxation time $\tau_{c}=1 / \Gamma=1 \mathrm{fm}$.

temperature and magnetic field dependence of $\zeta_{\perp}$ and $\zeta_{\|}$of the scalar and Dirac fluids.

\section{SUMMARY AND CONCLUSION}

In summary, we have performed a detailed calculation of the one-loop Kubo expressions of the five shear viscosity components and the two bulk viscosity components in the presence of an arbitrary background magnetic field $B$, where a general form of the thermomagnetic propagators are used based on the real time formalism of finite temperature field theory and the Schwinger proper time formalism. For this, we have taken two different systems(i) system of charged scalar bosons (spin 0) and (ii) system of charged Dirac fermions (spin $\frac{1}{2}$ ) — and have calculated the corresponding thermomagnetic spectral functions of the EMTs which is the imaginary part of the Fourier transform of the local EMT-EMT two-point correlator. Then viscous coefficients are estimated from these thermomagnetic spectral functions using the Kubo relations in the covariant tensor basis of Ref. [36].

In the absence of a magnetic field, it is quite standard that the one-loop Kubo expressions [38-42] of shear and bulk viscosity are exactly identical to the expression from the RTA of kinetic theory approach $[43,44]$, when we identify the inverse relation $\left(\Gamma=1 / \tau_{c}\right)$ between thermal width $\Gamma$ introduced in the propagators of Kubo method and the relaxation time $\tau_{c}$ in RTA method. However, in the presence of a magnetic field, this exact equality between the Kubo and RTA expressions has not been found as explored in present work. Recently, Refs. [6,8-12,14,17] have provided the RTA-based kinetic theory expression of the relativistic matter at finite magnetic field; of which Refs. [8,10-12,14,17] consider a general value of $B$ and Refs. [6,9] make a strong magnetic field approximation. In these calculations, five shear viscosity components and two bulk viscosity components become different in magnitude due to their $B$-dependent anisotropic factor, made by the two timescales: relaxation time $\tau_{c}$ and the inverse of synchrotron frequency $\tau_{B} \propto 1 / B$, where $B$ actually enters. If we compare those RTA expressions of viscosity components with the corresponding Kubo expressions, obtained in the present work, then one can find a rich magnetic-fielddependent structure, which probably reflects quantum field theoretical effects on viscosity expressions. One may think that a straightforward extension of Landau quantization of the RTA-based kinetic theory expression might be equal to our Kubo expressions, but this is not the case as the present work reveals; we have identified a rich vertex structure and an alternative entry of $\tau_{B}$ as an inverse of the difference 
between Landau quantized energies. Just like we realize the time period $(\tau)$ of radiation as the transition between the two energy levels in the hydrogen atom problem $\left(\tau^{-1}=\nu=\Delta E\right)$, here we can think of $\tau_{B}$ as the characteristic timescale for the transition between the two Landau levels and thus $\tau_{B}$ may be considered as the inverse of the energy difference of two Landau levels $\delta \omega_{l n}$.

Although the qualitative magnetic-field-dependent trends of RTA and Kubo expressions of viscosity components are quite similar, which can also be checked by recovering their isotropic picture, when one imposes the $B \rightarrow 0$ limit. Among the five components of shear viscosity $\eta_{n}(n=0,1,2,3,4), \eta_{1,2,3,4}$ components are completely originated due to the magnetic field and, therefore, they are disappeared in the $B \rightarrow 0$ limit, whereas $\eta_{0}$ only survives and merges with the isotropic value $\eta$, present at the $B=0$ picture. For the bulk viscosity components, isotropic value $\zeta$ at $B=0$ is split into two components along the parallel $\left(\zeta_{\|}\right)$and the perpendicular $\left(\zeta_{\perp}\right)$ direction with respect to the direction of the magnetic field, whose merging to the isotropic value is verified by imposing the numerical $B \rightarrow 0$ limit. The qualitative magnetic-field-dependent structure of the RTA and Kubo expressions is more or less same but we believe that the Kubo expressions proposed here are carrying a rich quantum field theoretical structure, which is probably missing in existing RTA expressions.

\section{ACKNOWLEDGMENTS}

The authors are thankful to Dr. Arghya Mukherjee and Sarthak Satapathy for carefully reading this article and for providing useful comments and suggestions. S. G. is funded by the Department of Higher Education, Government of West Bengal.

\section{APPENDIX A: CALCULATION OF THE EMT CORRELATORS}

In this appendix, we will sketch the calculation of the two-point correlation functions $\left\langle\mathcal{T}_{C} T^{\mu \nu}(x) T^{\alpha \beta}(y)\right\rangle_{11}$ for both the complex scalar and charged Dirac fields. Using Eq. (5), we have

$$
\begin{aligned}
\left\langle\mathcal{T}_{C} T_{\text {scalar }}^{\mu \nu}(x) T_{\text {scalar }}^{\alpha \beta}(y)\right\rangle_{11}= & \left\langle\mathcal{T}_{C}\left(\partial^{\mu} \phi^{\dagger}(x) \partial^{\nu} \phi(x)-\frac{1}{2} g^{\mu \nu} \mathcal{L}_{\text {scalar }}(x)\right)\left(\partial^{\alpha} \phi^{\dagger}(y) \partial^{\beta} \phi(y)-\frac{1}{2} g^{\alpha \beta} \mathcal{L}_{\text {scalar }}(y)\right)\right\rangle_{11} \\
& +(\mu \leftrightarrow \nu)+(\alpha \leftrightarrow \beta)+(\mu \leftrightarrow \nu, \alpha \leftrightarrow \beta) .
\end{aligned}
$$

Substituting $\mathcal{L}_{\text {scalar }}$ from Eq. (3) into Eq. (A1) and applying Wick's theorem [48], we arrive at

$$
\begin{array}{r}
\left\langle\mathcal{T}_{C} T_{\text {Scalar }}^{\mu \nu}(x) T_{\text {Scalar }}^{\alpha \beta}(y)\right\rangle_{11}=\left\langle\mathcal{T}_{C} \partial^{\mu} \phi^{\dagger}(x) \partial^{v} \phi(x) \partial^{\alpha} \phi^{\dagger}(y) \partial^{\beta} \phi(y)\right\rangle_{11}-\frac{1}{2} g^{\mu \nu}\left\langle\mathcal{T}_{C} \partial^{\sigma} \phi^{\dagger}(x) \partial_{\sigma} \phi(x) \partial^{\alpha} \phi^{\dagger}(y) \partial^{\beta} \phi(y)\right\rangle_{11} \\
+\frac{1}{2} g^{\mu v} m^{2}\left\langle\mathcal{T}_{C} \phi^{\dagger}(x) \phi(x) \partial^{\alpha} \phi^{\dagger}(y) \partial^{\beta} \phi(y)\right\rangle_{11}-\frac{1}{2} g^{\alpha \beta}\left\langle\mathcal{T}_{C} \partial^{\mu} \phi^{\dagger}(x) \partial^{v} \phi(x) \partial^{\sigma} \phi^{\dagger}(y) \partial_{\sigma} \phi(y)\right\rangle_{11} \\
+\frac{1}{2} g^{\alpha \beta} m^{2}\left\langle\mathcal{T}_{C} \partial^{\mu} \phi^{\dagger}(x) \partial^{v} \phi(x) \phi^{\dagger}(y) \phi(y)\right\rangle_{11}+\frac{1}{4} g^{\mu v} g^{\alpha \beta}\left\{\left\langle\mathcal{T}_{C} \partial^{\sigma} \phi^{\dagger}(x) \partial_{\sigma} \phi(x) \partial^{\rho} \phi^{\dagger}(y) \partial_{\rho} \phi(y)\right\rangle_{11}\right. \\
-m^{2}\left\langle\mathcal{T}_{C} \partial^{\sigma} \phi^{\dagger}(x) \partial_{\sigma} \phi(x) \phi^{\dagger}(y) \phi(y)\right\rangle_{11}-m^{2}\left\langle\mathcal{T}_{C} \phi^{\dagger}(x) \phi(x) \partial^{\sigma} \phi^{\dagger}(y) \partial_{\sigma} \phi(y)\right\rangle_{11} \\
\left.+m^{4}\left\langle\mathcal{T}_{C} \phi^{\dagger}(x) \phi(x) \phi^{\dagger}(y) \phi(y)\right\rangle_{11}\right\}+(\mu \leftrightarrow v)+(\alpha \leftrightarrow \beta)+(\mu \leftrightarrow v, \alpha \leftrightarrow \beta) .
\end{array}
$$

Simplification of the above equation yields

$$
\begin{aligned}
\left\langle\mathcal{T}_{C} T_{\text {scalar }}^{\mu \nu}(x) T_{\text {scalar }}^{\alpha \beta}(y)\right\rangle_{11}= & \partial_{x}^{\nu} \partial_{y}^{\alpha} D_{11}(x, y) \partial_{x}^{\mu} \partial_{y}^{\beta} D_{11}(y, x)-\frac{1}{2} g^{\mu \nu}\left\{\partial_{\sigma}^{x} \partial_{y}^{\alpha} D_{11}(x, y) \partial_{x}^{\sigma} \partial_{y}^{\beta} D_{11}(y, x)-m^{2} \partial_{y}^{\alpha} D_{11}(x, y) \partial_{y}^{\beta} D_{11}(y, x)\right\} \\
& -\frac{1}{2} g^{\alpha \beta}\left\{\partial_{x}^{\nu} \partial_{y}^{\sigma} D_{11}(x, y) \partial_{x}^{\mu} \partial_{\sigma}^{y} D_{11}(y, x)-m^{2} \partial_{x}^{\nu} D_{11}(x, y) \partial_{x}^{\mu} D_{11}(y, x)\right\} \\
& +\frac{1}{4} g^{\mu \nu} g^{\alpha \beta}\left\{\partial_{\sigma}^{x} \partial_{y}^{\rho} D_{11}(x, y) \partial_{x}^{\sigma} \partial_{\rho}^{y} D_{11}(y, x)-m^{2} \partial_{\sigma}^{x} D_{11}(x, y) \partial_{x}^{\sigma} D_{11}(y, x)-m^{2} \partial_{\sigma}^{y} D_{11}(x, y) \partial_{y}^{\sigma} D_{11}(y, x)\right. \\
& \left.+m^{4} D_{11}(x, y) D_{11}(y, x)\right\}+(\mu \leftrightarrow \nu)+(\alpha \leftrightarrow \beta)+(\mu \leftrightarrow \nu, \alpha \leftrightarrow \beta)
\end{aligned}
$$


where $\partial_{x}^{\mu} \equiv \frac{\partial}{\partial x_{\mu}}, \partial_{y}^{\mu} \equiv \frac{\partial}{\partial y_{\mu}}$, etc., and $D_{11}(x, y)=\left\langle\mathcal{T}_{C} \overline{\phi(x)} \phi^{\dagger}(y)\right\rangle_{11}$ is the 11-component of the real time free thermal scalar propagator in coordinate space. Since $D_{11}(x, y)=D_{11}(x-y)$ is translationally invariant, it can be Fourier transformed as

$$
D_{11}(x, y)=D_{11}(x-y)=\int \frac{d^{4} p}{(2 \pi)^{4}} e^{-i p \cdot(x-y)}\left(-i D_{11}(p ; m)\right)
$$

where $D_{11}(p ; m)$ is the 11-component of the momentum space real time free thermal scalar propagator whose explicit form reads $[45,46]$

$$
D_{11}(p ; m)=\left[\frac{-1}{p^{2}-m^{2}+i \epsilon}+\xi(p \cdot u) 2 \pi i \delta\left(p^{2}-m^{2}\right)\right]
$$

in which $u^{\mu}$ is the four-velocity of the medium, $\xi(x)=\Theta(x) f(x)+\Theta(-x) f(-x)$ and $f(x)=\left[e^{x / T}-1\right]^{-1}$ is the BoseEinstein distribution function at temperature $T$. In the LRF of the medium, $u_{\mathrm{LRF}}^{\mu} \equiv(1, \overrightarrow{0})$.

Substitution of Eq. (A4) into Eq. (A3) yields after some simplifications

$$
\left\langle\mathcal{T}_{C} T_{\text {scalar }}^{\mu \nu}(x) T_{\text {scalar }}^{\alpha \beta}(y)\right\rangle_{11}=-\iint \frac{d^{4} p}{(2 \pi)^{4}} \frac{d^{4} k}{(2 \pi)^{4}} e^{-i(x-y) \cdot(p-k)} D_{11}(p ; m) D_{11}(k ; m) \mathcal{N}_{\text {scalar }}^{\mu \nu \alpha \beta}(k, p),
$$

where

$$
\begin{aligned}
\mathcal{N}_{\text {scalar }}^{\mu \nu \alpha \beta}(k, p)= & k^{\mu} p^{\nu} p^{\alpha} k^{\beta}-\frac{1}{2}\left(p \cdot k-m^{2}\right)\left(g^{\mu \nu} p^{\alpha} k^{\beta}+g^{\alpha \beta} k^{\mu} p^{\nu}\right)+\frac{1}{4}\left(p \cdot k-m^{2}\right)^{2} g^{\mu \nu} g^{\alpha \beta} \\
& +(\mu \leftrightarrow \nu)+(\alpha \leftrightarrow \beta)+(\mu \leftrightarrow \nu, \alpha \leftrightarrow \beta) .
\end{aligned}
$$

In the calculation of viscous coefficients, we actually need the quantity $\mathcal{N}_{\text {scalar }}^{\mu \nu \alpha \beta}(k, k)$, which is easily followed from Eq. (A7) as

$$
\mathcal{N}_{\text {scalar }}^{\mu \nu \alpha \beta}(k, k)=4 k^{\mu} k^{\nu} k^{\alpha} k^{\beta}-2\left(k^{2}-m^{2}\right)\left(g^{\mu \nu} k^{\alpha} k^{\beta}+g^{\alpha \beta} k^{\mu} k^{\nu}\right)+\left(k^{2}-m^{2}\right)^{2} g^{\mu \nu} g^{\alpha \beta} .
$$

The calculation of EMT correlator for the Dirac field is done in similar ways. Using Eq. (6), we have

$$
\begin{aligned}
\left\langle\mathcal{T}_{C} T_{\text {Dirac }}^{\mu \nu}(x) T_{\text {Dirac }}^{\alpha \beta}(y)\right\rangle_{11}= & \left\langle\mathcal{T}_{C}\left(\frac{i}{4}\left(\bar{\psi}(x) \gamma^{\mu} \partial^{\nu} \psi(x)-\partial^{\nu} \bar{\psi}(x) \gamma^{\mu} \psi(x)\right)-\frac{1}{2} g^{\mu \nu} \mathcal{L}_{\text {Dirac }}(x)\right)\right. \\
& \left.\times\left(\frac{i}{4}\left(\bar{\psi}(y) \gamma^{\mu} \partial^{\nu} \psi(y)-\partial^{\nu} \bar{\psi}(y) \gamma^{\mu} \psi(y)\right)-\frac{1}{2} g^{\mu \nu} \mathcal{L}_{\text {Dirac }}(y)\right)\right\rangle_{11} \\
& +(\mu \leftrightarrow \nu)+(\alpha \leftrightarrow \beta)+(\mu \leftrightarrow \nu, \alpha \leftrightarrow \beta) .
\end{aligned}
$$

Substituting $\mathcal{L}_{\text {Dirac }}$ from Eq. (4) into Eq. (A9) and applying Wick's theorem [48], we arrive at 


$$
\begin{aligned}
& \left\langle\mathcal{T}_{C} T_{\text {Dirac }}^{\mu \nu}(x) T_{\text {Dirac }}^{\alpha \beta}(y)\right\rangle_{11}=-\frac{1}{16}\left\{\left\langle\mathcal{T}_{C} \bar{\psi}(x) \gamma^{\mu} \partial^{v} \overline{\psi(x) \bar{\psi}}(y) \gamma^{\alpha} \partial^{\beta} \psi(y)\right\rangle_{11}-\left\langle\mathcal{T}_{C} \bar{\psi}(x) \gamma^{\mu} \partial^{v} \psi(x) \partial^{\beta} \bar{\psi}(y) \gamma^{\alpha} \psi(y)\right\rangle_{11}\right. \\
& \left.-\left\langle\mathcal{T}_{C} \partial^{\nu} \bar{\psi}(x) \gamma^{\mu} \psi(x) \bar{\psi}(y) \gamma^{\alpha} \partial^{\beta} \psi(y)\right\rangle_{11}+\left\langle\mathcal{T}_{C} \partial^{v} \bar{\psi}(x) \gamma^{\mu} \psi(x) \partial^{\beta} \bar{\psi}(y) \gamma^{\alpha} \psi(y)\right\rangle_{11}\right\} \\
& +\frac{1}{16} g^{\mu \nu}\left\{\left\langle\mathcal{T}_{C} \bar{\psi}(x) \gamma^{\sigma} \partial_{\sigma} \overline{\psi(x) \bar{\psi}}(y) \gamma^{\alpha} \partial^{\beta} \psi(y)\right\rangle_{11}-\left\langle\mathcal{T}_{C} \bar{\psi}(x) \gamma^{\sigma} \partial_{\sigma} \overline{\psi(x) \partial^{\beta} \bar{\psi}}(y) \gamma^{\alpha} \psi(y)\right\rangle_{11}\right.
\end{aligned}
$$

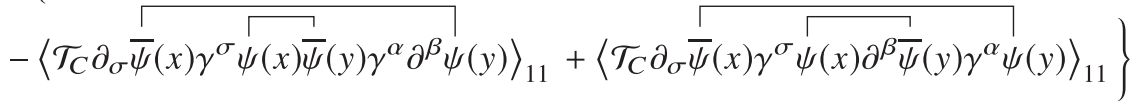

$$
\begin{aligned}
& +\frac{i}{8} g^{\mu \nu} m\left\{\left\langle\mathcal{T}_{C} \bar{\psi}(x) \overline{\psi(x) \bar{\psi}}(y) \gamma^{\alpha} \partial^{\beta} \psi(y)\right\rangle_{11}-\left\langle\mathcal{T}_{C} \bar{\psi} \bar{\psi}(x) \psi(x) \partial^{\beta} \bar{\psi}(y) \gamma^{\alpha} \psi(y)\right\rangle_{11}\right\} \\
& +\frac{1}{16} g^{\alpha \beta}\left\{\left\langle\mathcal{T}_{C} \bar{\psi}(x) \gamma^{\mu} \partial^{v} \overline{\psi(x) \bar{\psi}}(y) \gamma^{\sigma} \partial_{\sigma} \psi(y)\right\rangle_{11}-\left\langle\mathcal{T}_{C} \partial^{v} \bar{\psi}(x) \gamma^{\mu} \bar{\psi}(x) \bar{\psi}(y) \gamma^{\sigma} \partial_{\sigma} \psi(y)\right\rangle_{11}\right. \\
& \left.-\left\langle\mathcal{T}_{C} \bar{\psi}(x) \gamma^{\mu} \partial^{v}{ }_{\psi}(x) \partial_{\sigma} \bar{\psi}(y) \gamma^{\sigma} \psi(y)\right\rangle_{11}+\left\langle\mathcal{T}_{C} \partial^{v} \bar{\psi}(x) \gamma^{\mu}{ }_{\psi(x) \partial_{\sigma}} \bar{\psi}(y) \gamma^{\sigma} \psi(y)\right\rangle_{11}\right\} \\
& +\frac{i}{8} g^{\alpha \beta} m\left\{\left\langle\mathcal{T}_{C} \bar{\psi}(x) \gamma^{\mu} \partial^{v} \overline{\psi(x) \bar{\psi}}(y) \psi(y)\right\rangle_{11}-\left\langle\mathcal{T}_{C} \partial^{\nu} \bar{\psi}(x) \gamma^{\mu} \overline{\psi(x) \bar{\psi}}(y) \psi(y)\right\rangle_{11}\right\} \\
& -\frac{1}{16} g^{\mu v} g^{\alpha \beta}\left\{\left\langle\mathcal{T}_{C} \bar{\psi}(x) \gamma^{\sigma} \partial_{\sigma} \bar{\psi}(x) \bar{\psi}(y) \gamma^{\rho} \partial_{\rho} \psi(y)\right\rangle_{11}-\left\langle\mathcal{T}_{C} \partial_{\sigma} \bar{\psi}(x) \gamma^{\sigma} \bar{\psi}(x) \bar{\psi}(y) \gamma^{\rho} \partial_{\rho} \psi(y)\right\rangle_{11}\right. \\
& \left.-\left\langle\mathcal{T}_{C} \bar{\psi}(x) \gamma^{\sigma} \partial_{\sigma} \psi(x) \partial_{\rho} \bar{\psi}(y) \gamma^{\rho} \psi(y)\right\rangle_{11}+\left\langle\mathcal{T}_{C} \partial_{\sigma} \bar{\psi}(x) \gamma^{\sigma} \overline{\psi(x) \partial_{\rho} \bar{\psi}}(y) \gamma^{\rho} \psi(y)\right\rangle_{11}\right\} \\
& -\frac{i}{8} g^{\mu v} g^{\alpha \beta} m\left\{\left\langle\mathcal{T}_{C} \bar{\psi}(x) \gamma^{\sigma} \partial_{\sigma} \psi(x) \bar{\psi}(y) \psi(y)\right\rangle_{11}-\left\langle\mathcal{T}_{C} \partial_{\sigma} \bar{\psi}(x) \gamma^{\sigma} \bar{\psi}(x) \bar{\psi}(y) \psi(y)\right\rangle_{11}\right. \\
& \left.-\left\langle\mathcal{T}_{C} \bar{\psi}(x) \psi(x) \partial_{\rho} \bar{\psi}(y) \gamma^{\rho} \psi(y)\right\rangle_{11}+\left\langle\mathcal{T}_{C} \bar{\psi}(x) \bar{\psi}(x) \bar{\psi}(y) \gamma^{\rho} \partial_{\rho} \psi(y)\right\rangle_{11}\right\} \\
& +\frac{1}{4} g^{\mu v} g^{\alpha \beta} m^{2}\left\langle\mathcal{T}_{C} \bar{\psi}(x) \overline{\psi(x) \bar{\psi}}(y) \psi(y)\right\rangle_{11}+(\mu \leftrightarrow v)+(\alpha \leftrightarrow \beta)+(\mu \leftrightarrow v, \alpha \leftrightarrow \beta) .
\end{aligned}
$$

Simplification of the above equation yields

$$
\begin{aligned}
\left\langle\mathcal{T}_{C} T_{\text {Dirac }}^{\mu \nu}(x) T_{\text {Dirac }}^{\alpha \beta}(y)\right\rangle_{11}= & \frac{1}{16} \operatorname{Tr}\left\{\gamma^{\mu} \partial_{x}^{\nu} S_{11}(x, y) \gamma^{\alpha} \partial_{y}^{\beta} S_{11}(y, x)-\gamma^{\mu} \partial_{x}^{\nu} \partial_{y}^{\beta} S_{11}(x, y) \gamma^{\alpha} S_{11}(y, x)\right. \\
& \left.-\gamma^{\mu} S_{11}(x, y) \gamma^{\alpha} \partial_{x}^{\nu} \partial_{y}^{\beta} S_{11}(y, x)+\gamma^{\mu} \partial_{y}^{\beta} S_{11}(x, y) \gamma^{\alpha} \partial_{x}^{\nu} S_{11}(y, x)\right\} \\
& -\frac{1}{16} g^{\mu \nu} \operatorname{Tr}\left\{\gamma^{\sigma} \partial_{\sigma}^{x} S_{11}(x, y) \gamma^{\alpha} \partial_{y}^{\beta} S_{11}(y, x)-\gamma^{\sigma} \partial_{\sigma}^{x} \partial_{y}^{\beta} S_{11}(x, y) \gamma^{\alpha} S_{11}(y, x)\right. \\
& \left.-\gamma^{\sigma} S_{11}(x, y) \gamma^{\alpha} \partial_{\sigma}^{x} \partial_{y}^{\beta} S_{11}(y, x)+\gamma^{\sigma} \partial_{y}^{\beta} S_{11}(x, y) \gamma^{\alpha} \partial_{\sigma}^{x} S_{11}(y, x)\right\} \\
& -\frac{i}{8} g^{\mu \nu} m \operatorname{Tr}\left\{S_{11}(x, y) \gamma^{\alpha} \partial_{y}^{\beta} S_{11}(y, x)-\partial_{y}^{\beta} S_{11}(x, y) \gamma^{\alpha} S_{11}(y, x)\right\} \\
& -\frac{1}{16} g^{\alpha \beta} \operatorname{Tr}\left\{\gamma^{\mu} \partial_{x}^{\nu} S_{11}(x, y) \gamma^{\sigma} \partial_{\sigma}^{y} S_{11}(y, x)-\gamma^{\mu} S_{11}(x, y) \gamma^{\sigma} \partial_{x}^{\nu} \partial_{\sigma}^{y} S_{11}(y, x)\right. \\
& \left.-\gamma^{\mu} \partial_{x}^{\nu} \partial_{\sigma}^{y} S_{11}(x, y) \gamma^{\sigma} S_{11}(y, x)+\gamma^{\mu} \partial_{\sigma}^{y} S_{11}(x, y) \gamma^{\sigma} \partial_{x}^{\nu} S_{11}(y, x)\right\} \\
& -\frac{i}{8} g^{\alpha \beta} m \operatorname{Tr}\left\{\gamma^{\mu} \partial_{x}^{\nu} S_{11}(x, y) S_{11}(y, x)-\gamma^{\mu} S_{11}(x, y) \partial_{x}^{\nu} S_{11}(y, x)\right\}
\end{aligned}
$$




$$
\begin{aligned}
& +\frac{1}{16} g^{\mu \nu} g^{\alpha \beta} \operatorname{Tr}\left\{\gamma^{\sigma} \partial_{\sigma}^{x} S_{11}(x, y) \gamma^{\rho} \partial_{\rho}^{y} S_{11}(y, x)-\gamma^{\sigma} \partial_{\sigma}^{x} \partial_{\rho}^{y} S_{11}(x, y) \gamma^{\rho} S_{11}(y, x)\right. \\
& \left.-\gamma^{\sigma} S_{11}(x, y) \gamma^{\rho} \partial_{\sigma}^{x} \partial_{\rho}^{y} S_{11}(y, x)+\gamma^{\sigma} \partial_{\rho}^{y} S_{11}(x, y) \gamma^{\rho} \partial_{\sigma}^{x} S_{11}(y, x)\right\} \\
& +\frac{i}{8} g^{\mu \nu} g^{\alpha \beta} m \operatorname{Tr}\left\{\gamma^{\sigma} \partial_{\sigma}^{x} S_{11}(x, y) S_{11}(y, x)-\gamma^{\sigma} S_{11}(x, y) \partial_{\sigma}^{x} S_{11}(y, x)-\partial_{\rho}^{y} S_{11}(x, y) \gamma^{\rho} S_{11}(y, x)\right. \\
& \left.+S_{11}(x, y) \gamma^{\rho} \partial_{\rho}^{y} S_{11}(y, x)\right\}-\frac{1}{4} g^{\mu \nu} g^{\alpha \beta} m^{2} \operatorname{Tr}\left\{S_{11}(x, y) S_{11}(y, x)\right\} \\
& +(\mu \leftrightarrow \nu)+(\alpha \leftrightarrow \beta)+(\mu \leftrightarrow \nu, \alpha \leftrightarrow \beta),
\end{aligned}
$$

where $S_{11}(x, y)=\left\langle\mathcal{T}_{C} \psi(x) \bar{\psi}(y)\right\rangle_{11}$ is the 11-component of the real time free thermal Dirac propagator in coordinate space. It may be noted that the above expression is valid even if the field $\psi$ is a multiplet, in which case the traces in the above equation have to be taken over all the spaces belonging to the multiplet in addition to the Dirac space. Similar to the scalar propagator, $S_{11}(x, y)=S_{11}(x-y)$ is translationally invariant and it can be Fourier transformed as

$$
S_{11}(x, y)=S_{11}(x-y)=\int \frac{d^{4} p}{(2 \pi)^{4}} e^{-i p \cdot(x-y)}\left(-i S_{11}(p)\right)
$$

where $S_{11}(p)$ is the 11-component of the momentum space real time free thermal Dirac propagator whose explicit form reads $[45,46]$

$$
S_{11}(p)=(\not p+m)\left[\frac{-1}{p^{2}-m^{2}+i \epsilon}-\tilde{\xi}(p \cdot u) 2 \pi i \delta\left(p^{2}-m^{2}\right)\right]
$$

in which $\tilde{\xi}(x)=\Theta(x) \tilde{f}(x)+\Theta(-x) \tilde{f}(-x)$ and $\tilde{f}(x)=\left[e^{x / T}+1\right]^{-1}$ is the Fermi-Dirac distribution function at temperature $T$.

Substitution of Eq. (A12) into Eq. (A11) yields after some simplifications

$$
\begin{aligned}
\left\langle\mathcal{T}_{C} T_{\text {Dirac }}^{\mu \nu}(x) T_{\text {Dirac }}^{\alpha \beta}(y)\right\rangle_{11}= & \iint \frac{d^{4} p}{(2 \pi)^{4}} \frac{d^{4} k}{(2 \pi)^{4}} e^{-i(x-y) \cdot(p-k)}\left\{\frac{1}{16}\left(p^{\nu} k^{\beta}+k^{\nu} p^{\beta}+p^{\nu} p^{\beta}+k^{\nu} k^{\beta}\right) \operatorname{Tr}\left(\gamma^{\mu} S_{11}(p) \gamma^{\alpha} S_{11}(k)\right)\right. \\
& -\frac{1}{16} g^{\mu \nu}\left(p_{\sigma} k^{\beta}+k_{\sigma} p^{\beta}+p_{\sigma} p^{\beta}+k_{\sigma} k^{\beta}\right) \operatorname{Tr}\left(\gamma^{\sigma} S_{11}(p) \gamma^{\alpha} S_{11}(k)\right)+\frac{1}{8} g^{\mu \nu} m\left(p^{\beta}+k^{\beta}\right) \operatorname{Tr}\left(S_{11}(p) \gamma^{\alpha} S_{11}(k)\right) \\
& -\frac{1}{16} g^{\alpha \beta}\left(p^{\nu} k_{\sigma}+k^{\nu} p_{\sigma}+p^{\nu} p_{\sigma}+k^{\nu} k_{\sigma}\right) \operatorname{Tr}\left(\gamma^{\mu} S_{11}(p) \gamma^{\sigma} S_{11}(k)\right)+\frac{1}{8} g^{\alpha \beta} m\left(p^{\nu}+k^{\nu}\right) \operatorname{Tr}\left(\gamma^{\mu} S_{11}(p) S_{11}(k)\right) \\
& +\frac{1}{16} g^{\mu \nu} g^{\alpha \beta}\left(p_{\sigma} k_{\rho}+k_{\sigma} p_{\rho}+p_{\sigma} p_{\rho}+k_{\sigma} k_{\rho}\right) \operatorname{Tr}\left(\gamma^{\sigma} S_{11}(p) \gamma^{\rho} S_{11}(k)\right) \\
& -\frac{1}{8} g^{\mu \nu} g^{\alpha \beta} m\left(p_{\sigma}+k_{\sigma}\right)\left\{\operatorname{Tr}\left(S_{11}(p) \gamma^{\sigma} S_{11}(k)\right)+\operatorname{Tr}\left(\gamma^{\sigma} S_{11}(p) S_{11}(k)\right)\right\} \\
& \left.+\frac{1}{4} g^{\mu \nu} g^{\alpha \beta} m^{2} \operatorname{Tr}\left(S_{11}(p) S_{11}(k)\right)\right\}+(\mu \leftrightarrow \nu)+(\alpha \leftrightarrow \beta)+(\mu \leftrightarrow \nu, \alpha \leftrightarrow \beta) .
\end{aligned}
$$

Substituting $S_{11}(p)$ from Eq. (A13) into Eq. (A14) followed by evaluating the traces over the Dirac matrices and simplifying, we arrive at

$$
\left\langle\mathcal{T}_{C} T_{\text {Dirac }}^{\mu \nu}(x) T_{\text {Dirac }}^{\alpha \beta}(y)\right\rangle_{11}=-\iint \frac{d^{4} p}{(2 \pi)^{4}} \frac{d^{4} k}{(2 \pi)^{4}} e^{-i(x-y) \cdot(p-k)} \tilde{D}_{11}(p ; m) \tilde{D}_{11}(k ; m) \mathcal{N}_{\text {Dirac }}^{\mu \nu \alpha \beta}(k, p),
$$

where

$$
\tilde{D}_{11}(p ; m)=\left[\frac{-1}{p^{2}-m^{2}+i \epsilon}-\tilde{\xi}(p \cdot u) 2 \pi i \delta\left(p^{2}-m^{2}\right)\right]
$$

and 


$$
\begin{aligned}
\mathcal{N}_{\text {Dirac }}^{\mu \nu \alpha \beta}(k, p)= & \frac{1}{4}\left[-\left(k^{\mu} p^{\alpha}+p^{\mu} k^{\alpha}\right)\left(k^{\beta}+p^{\beta}\right)\left(k^{\nu}+p^{\nu}\right)+g^{\mu \alpha}\left(k^{\beta}+p^{\beta}\right)\left(k^{\nu}+p^{\nu}\right)\left(k \cdot p-m^{2}\right)\right. \\
& +g^{\mu \nu}\left(k^{\beta}+p^{\beta}\right)\left\{\left(p^{2}-m^{2}\right) k^{\alpha}+\left(k^{2}-m^{2}\right) p^{\alpha}\right\}+g^{\alpha \beta}\left(k^{\nu}+p^{\nu}\right)\left\{\left(p^{2}-m^{2}\right) k^{\mu}+\left(k^{2}-m^{2}\right) p^{\mu}\right\} \\
& \left.-g^{\mu \nu} g^{\alpha \beta}\left\{2\left(k^{2}-m^{2}\right)\left(p^{2}-m^{2}\right)+\left(k \cdot p-m^{2}\right)\left(k^{2}-m^{2}+p^{2}-m^{2}\right)\right\}\right] \\
& +(\mu \leftrightarrow \nu)+(\alpha \leftrightarrow \beta)+(\mu \leftrightarrow \nu, \alpha \leftrightarrow \beta) .
\end{aligned}
$$

In the calculation of viscous coefficients, we actually need the quantity $\mathcal{N}_{\text {Dirac }}^{\mu \nu \alpha \beta}(k, k)$, which is easily followed from Eq. (A17) as

$$
\begin{aligned}
\mathcal{N}_{\text {Dirac }}^{\mu \nu \alpha \beta}(k, k)= & -8 k^{\mu} k^{\nu} k^{\alpha} k^{\beta}+\left(k^{2}-m^{2}\right)\left\{g^{\mu \alpha} k^{\nu} k^{\beta}+g^{\nu \alpha} k^{\mu} k^{\beta}+g^{\mu \beta} k^{\nu} k^{\alpha}+g^{\nu \beta} k^{\mu} k^{\alpha}\right. \\
& \left.+4 g^{\mu \nu} k^{\alpha} k^{\beta}+4 g^{\alpha \beta} k^{\mu} k^{\nu}\right\}-4\left(k^{2}-m^{2}\right)^{2} g^{\mu \nu} g^{\alpha \beta} .
\end{aligned}
$$

\section{APPENDIX B: CALCULATION OF THE EMT CORRELATORS IN THE PRESENCE OF AN EXTERNAL MAGNETIC FIELD}

In this appendix, we will sketch the calculation of the two-point correlation functions $\left\langle\mathcal{T}_{C} T^{\mu \nu}(x) T^{\alpha \beta}(y)\right\rangle_{11}^{B}$ in the presence of a constant external magnetic field for both the complex scalar and charged Dirac fields. Proceeding along similar lines as done in Appendix A, Eq. (A3) for the scalar field now modifies in the presence of a magnetic field as

$$
\begin{aligned}
\left\langle\mathcal{T}_{C} T_{\text {scalar }}^{\mu \nu}(x) T_{\text {scalar }}^{\alpha \beta}(y)\right\rangle_{11}^{B}= & D_{x}^{\nu} D_{y}^{* \alpha} D_{11}^{B}(x, y) D_{x}^{* \mu} D_{y}^{\beta} D_{11}^{B}(y, x)-\frac{1}{2} g^{\mu \nu}\left\{D_{\sigma}^{x} D_{y}^{* \alpha} D_{11}^{B}(x, y) D_{x}^{* \sigma} D_{y}^{\beta} D_{11}^{B}(y, x)\right. \\
& \left.-m^{2} D_{y}^{* \alpha} D_{11}^{B}(x, y) D_{y}^{\beta} D_{11}^{B}(y, x)\right\} \\
& -\frac{1}{2} g^{\alpha \beta}\left\{D_{x}^{\nu} D_{y}^{* \sigma} D_{11}^{B}(x, y) D_{x}^{* \mu} D_{\sigma}^{y} D_{11}^{B}(y, x)-m^{2} D_{x}^{\nu} D_{11}^{B}(x, y) D_{x}^{* \mu} D_{11}^{B}(y, x)\right\} \\
& +\frac{1}{4} g^{\mu \nu} g^{\alpha \beta}\left\{D_{\sigma}^{x} D_{y}^{* \rho} D_{11}^{B}(x, y) D_{x}^{* \sigma} D_{\rho}^{y} D_{11}^{B}(y, x)-m^{2} D_{\sigma}^{x} D_{11}^{B}(x, y) D_{x}^{* \sigma} D_{11}^{B}(y, x)\right. \\
& \left.-m^{2} \partial_{\sigma}^{y} D_{11}^{B}(x, y) D_{y}^{* \sigma} D_{11}^{B}(y, x)+m^{4} D_{11}^{B}(x, y) D_{11}^{B}(y, x)\right\} \\
& +(\mu \leftrightarrow \nu)+(\alpha \leftrightarrow \beta)+(\mu \leftrightarrow \nu, \alpha \leftrightarrow \beta),
\end{aligned}
$$

where $\quad D_{x}^{\mu} \equiv \partial_{x}^{\mu}+i e A_{\mathrm{ext}}^{\mu}(x), \quad D_{x}^{* \mu} \equiv \partial_{x}^{\mu}-i e A_{\mathrm{ext}}^{\mu}(x)$, $D_{y}^{\mu} \equiv \partial_{y}^{\mu}+i e A_{\mathrm{ext}}^{\mu}(y), \quad D_{y}^{* \mu} \equiv \partial_{y}^{\mu}-i e A_{\mathrm{ext}}^{\mu}(y), \quad$ etc., $\quad$ and $D_{11}^{B}(x, y)=\left\langle\mathcal{T}_{C} \overline{\phi(x) \phi^{\dagger}}(y)\right\rangle_{11}^{B}$ is the 11-component of the real time free thermomagnetic scalar propagator in coordinate space. In contrast to the zero magnetic field case, the thermomagnetic propagator is no longer translationally invariant $D_{11}^{B}(x, y)=\Phi(x, y) D_{11}^{B}(x-y)$ and it contains the gauge-dependent phase factor $\Phi(x, y)$ which explicitly breaks the translational invariance. However, the translationally invariant piece $D_{11}^{B}(x-y)$ can be Fourier transformed and the thermomagnetic propagator can be written as

$$
D_{11}^{B}(x-y)=\int \frac{d^{4} p}{(2 \pi)^{4}} e^{-i p \cdot(x-y)}\left(-i D_{11}^{B}(p)\right),
$$

where $D_{11}^{B}(p)$ is the 11-component of the momentum space real time free thermomagnetic scalar propagator whose explicit form reads [63]

$$
D_{11}^{B}(p)=\sum_{l=0}^{\infty} 2(-1)^{l} e^{-\alpha_{p}} L_{l}\left(2 \alpha_{p}\right) D_{11}\left(p_{\|}, m_{l}\right)
$$

in which $l$ is the Landau level index, $\alpha_{p}=-\frac{p_{\perp}^{2}}{e B} \geq 0, m_{l}=$ $\sqrt{m^{2}+(2 l+1-2 s) e B}=\sqrt{m^{2}+(2 l+1) e B}$ is the "Landau-level-dependent effective mass," $s$ is the spin of the particle (for scalar field $s=0$ ) and $D_{11}$ is defined in Eq. (A5). Due to the external magnetic field in the $\hat{z}$ direction, the decomposition of any four vector $k^{\mu}$ is done as $k=\left(k_{\|}+k_{\perp}\right)$, where $k_{\|}^{\mu}=g_{\|}^{\mu \nu} k_{\nu}$ and $k_{\perp}^{\mu}=g_{\perp}^{\mu \nu} k_{\nu}$; the corresponding decomposition of the metric tensor reads $g^{\mu \nu}=\left(g_{\|}^{\mu \nu}+g_{\perp}^{\mu \nu}\right)$ with $g_{\|}^{\mu \nu}=\operatorname{diag}(1,0,0,-1)$ and $g_{\perp}^{\mu \nu}=\operatorname{diag}(0,-1,-1,0)$. Note that, in our convention, $k_{\perp}^{\mu}$ is a spacelike vector with $k_{\perp}^{2}=-\left(k_{x}^{2}+k_{y}^{2}\right)<0$.

Due to the presence of the phase factor $\Phi(x, y)$ in the propagator, it may seem that the quantity $\left\langle\mathcal{T}_{C} T_{\text {scalar }}^{\mu \nu}(x) T_{\text {scalar }}^{\alpha \beta}(y)\right\rangle_{11}^{B}$ in Eq. (B1) is not translationally invariant. Fortunately this is not the case. To see this, we 
first note that the gauge-dependent phase factor is given by [63]

$$
\Phi(x, y)=\exp \left[i e \int_{x}^{y} d x_{\mu}^{\prime} A_{\mathrm{ext}}^{\mu}\left(x^{\prime}\right)\right] .
$$

Differentiating the above equations separately with respect to $x$ and $y$ using the Leibniz rule yields

$$
\begin{gathered}
\partial_{x}^{\mu} \Phi(x, y)=\Phi(x, y)\left\{-i e A_{\mathrm{ext}}^{\mu}(x)\right\} \\
\partial_{y}^{\mu} \Phi(x, y)=\Phi(x, y)\left\{i e A_{\mathrm{ext}}^{\mu}(x)\right\} .
\end{gathered}
$$

The above two equations can be rewritten as

$$
D_{x}^{\mu} \Phi(x, y)=D_{y}^{* \mu} \Phi(x, y)=0 .
$$

Using Eq. (B7), it is easy to see that

$$
\begin{aligned}
D_{x}^{\mu} D_{11}^{B}(x, y) & =D_{x}^{\mu}\left[\Phi(x, y) D_{11}^{B}(x-y)\right] \\
& =\Phi(x, y) \partial_{x}^{\mu} D_{11}^{B}(x-y), \\
D_{y}^{* \mu} D_{11}^{B}(x, y) & =D_{y}^{* \mu}\left[\Phi(x, y) D_{11}^{B}(x-y)\right] \\
& =\Phi(x, y) \partial_{y}^{\mu} D_{11}^{B}(x-y) .
\end{aligned}
$$

We now use Eqs. (B8) and (B9) to simplify Eq. (B1) and get

$$
\begin{aligned}
\left\langle\mathcal{T}_{C} T_{\text {scalar }}^{\mu \nu}(x) T_{\text {scalar }}^{\alpha \beta}(y)\right\rangle_{11}^{B}= & \Phi(x, y) \Phi(y, x)\left[\partial_{x}^{\nu} \partial_{y}^{\alpha} D_{11}^{B}(x-y) \partial_{x}^{\mu} \partial_{y}^{\beta} D_{11}^{B}(y-x)\right. \\
& -\frac{1}{2} g^{\mu \nu}\left\{\partial_{\sigma}^{x} \partial_{y}^{\alpha} D_{11}^{B}(x-y) \partial_{x}^{\sigma} \partial_{y}^{\beta} D_{11}^{B}(y-x)-m^{2} \partial_{y}^{\alpha} D_{11}^{B}(x-y) \partial_{y}^{\beta} D_{11}^{B}(y-x)\right\} \\
& -\frac{1}{2} g^{\alpha \beta}\left\{\partial_{x}^{\nu} \partial_{y}^{\sigma} D_{11}^{B}(x-y) \partial_{x}^{\mu} \partial_{\sigma}^{y} D_{11}^{B}(y-x)-m^{2} \partial_{x}^{\nu} D_{11}^{B}(x-y) \partial_{x}^{\mu} D_{11}^{B}(y-x)\right\} \\
& +\frac{1}{4} g^{\mu \nu} g^{\alpha \beta}\left\{\partial_{\sigma}^{x} \partial_{y}^{\rho} D_{11}^{B}(x-y) \partial_{x}^{\sigma} \partial_{\rho}^{y} D_{11}^{B}(y-x)-m^{2} \partial_{\sigma}^{x} D_{11}^{B}(x-y) \partial_{x}^{\sigma} D_{11}^{B}(y-x)\right. \\
& \left.\left.-m^{2} \partial_{\sigma}^{y} D_{11}^{B}(x-y) \partial_{y}^{\sigma} D_{11}^{B}(y-x)+m^{4} D_{11}^{B}(x-y) D_{11}^{B}(y-x)\right\}\right] \\
& +(\mu \leftrightarrow \nu)+(\alpha \leftrightarrow \beta)+(\mu \leftrightarrow \nu, \alpha \leftrightarrow \beta) .
\end{aligned}
$$

Since the phase factor given in Eq. (B4) satisfies the relation $\Phi(x, y) \Phi(y, x)=1$, we notice that the phase factor in Eq. (B10) is completely canceled out and the quantity $\left\langle\mathcal{T}_{C} T_{\text {scalar }}^{\mu \nu}(x) T_{\text {scalar }}^{\alpha \beta}(y)\right\rangle_{11}^{B}$ is indeed translationally invariant and gauge independent. This type of cancellation of the phase factor for the loops containing particles with equal charges is well known $[51,53,63]$. If we now compare
Eq. (B10) with Eq. (A3), we notice that the expression of EMT correlator at nonzero magnetic field is identical to the same at zero magnetic field, except the thermal propagator has to be replaced by the translationally invariant piece of the thermomagnetic propagator.

Let us now substitute Eq. (B2) into Eq. (B10) and we get after some simplifications

$$
\left\langle\mathcal{T}_{C} T_{\text {scalar }}^{\mu \nu}(x) T_{\text {scalar }}^{\alpha \beta}(y)\right\rangle_{11}^{B}=-\iint \frac{d^{4} p}{(2 \pi)^{4}} \frac{d^{4} k}{(2 \pi)^{4}} e^{-i(x-y) \cdot(p-k)} \sum_{l=0}^{\infty} \sum_{n=0}^{\infty} D_{11}\left(p_{\|} ; m_{n}\right) D_{11}\left(k_{\|} ; m_{l}\right) \mathcal{N}_{l n ; \text { scalar }}^{\mu \nu \alpha \beta}(k, p),
$$

where

$$
\mathcal{N}_{l n ; \text { scalar }}^{\mu \nu \alpha \beta}(k, p)=4(-1)^{l+n} e^{-\alpha_{k}-\alpha_{p}} L_{l}\left(2 \alpha_{k}\right) L_{n}\left(2 \alpha_{p}\right) \mathcal{N}_{\text {scalar }}^{\mu \nu \alpha \beta}(k, p)
$$

in which $\mathcal{N}_{\text {scalar }}^{\mu \nu \alpha \beta}(k, p)$ is defined in Eq. (A7).

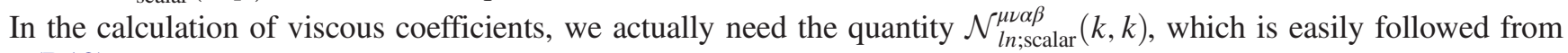
Eq. (B12) as

$$
\mathcal{N}_{l n ; \text { scalar }}^{\mu \nu \alpha \beta}(k, k)=4 \mathcal{A}_{l n}\left(k_{\perp}^{2}\right)\left\{4 k^{\mu} k^{\nu} k^{\alpha} k^{\beta}-2\left(k^{2}-m^{2}\right)\left(g^{\mu \nu} k^{\alpha} k^{\beta}+g^{\alpha \beta} k^{\mu} k^{\nu}\right)+\left(k^{2}-m^{2}\right)^{2} g^{\mu \nu} g^{\alpha \beta}\right\}
$$

in which

$$
\mathcal{A}_{l n}\left(k_{\perp}^{2}\right)=(-1)^{l+n} e^{-2 \alpha_{k}} L_{l}\left(2 \alpha_{k}\right) L_{n}\left(2 \alpha_{k}\right) .
$$


The calculation of the EMT correlator $\left\langle\mathcal{T}_{C} T_{\text {Dirac }}^{\mu \nu}(x) T_{\text {Dirac }}^{\alpha \beta}(y)\right\rangle_{11}^{B}$ for the Dirac field in the presence of an external magnetic field can be done in a similar fashion as done for the scalar field. In this case, the zero magnetic field expression of Eq. (A14) modifies to

$$
\begin{aligned}
\left\langle\mathcal{T}_{C} T_{\text {Dirac }}^{\mu \nu}(x) T_{\text {Dirac }}^{\alpha \beta}(y)\right\rangle_{11}^{B}= & \iint \frac{d^{4} p}{(2 \pi)^{4}} \frac{d^{4} k}{(2 \pi)^{4}} e^{-i(x-y) \cdot(p-k)}\left\{\frac{1}{16}\left(p^{\nu} k^{\beta}+k^{\nu} p^{\beta}+p^{\nu} p^{\beta}+k^{\nu} k^{\beta}\right) \operatorname{Tr}\left(\gamma^{\mu} S_{11}^{B}(p) \gamma^{\alpha} S_{11}^{B}(k)\right)\right. \\
& -\frac{1}{16} g^{\mu \nu}\left(p_{\sigma} k^{\beta}+k_{\sigma} p^{\beta}+p_{\sigma} p^{\beta}+k_{\sigma} k^{\beta}\right) \operatorname{Tr}\left(\gamma^{\sigma} S_{11}^{B}(p) \gamma^{\alpha} S_{11}^{B}(k)\right)+\frac{1}{8} g^{\mu \nu} m\left(p^{\beta}+k^{\beta}\right) \operatorname{Tr}\left(S_{11}^{B}(p) \gamma^{\alpha} S_{11}^{B}(k)\right) \\
& -\frac{1}{16} g^{\alpha \beta}\left(p^{\nu} k_{\sigma}+k^{\nu} p_{\sigma}+p^{\nu} p_{\sigma}+k^{\nu} k_{\sigma}\right) \operatorname{Tr}\left(\gamma^{\mu} S_{11}^{B}(p) \gamma^{\sigma} S_{11}^{B}(k)\right)+\frac{1}{8} g^{\alpha \beta} m\left(p^{\nu}+k^{\nu}\right) \operatorname{Tr}\left(\gamma^{\mu} S_{11}^{B}(p) S_{11}^{B}(k)\right) \\
& +\frac{1}{16} g^{\mu \nu} g^{\alpha \beta}\left(p_{\sigma} k_{\rho}+k_{\sigma} p_{\rho}+p_{\sigma} p_{\rho}+k_{\sigma} k_{\rho}\right) \operatorname{Tr}\left(\gamma^{\sigma} S_{11}^{B}(p) \gamma^{\rho} S_{11}^{B}(k)\right) \\
& -\frac{1}{8} g^{\mu \nu} g^{\alpha \beta} m\left(p_{\sigma}+k_{\sigma}\right)\left\{\operatorname{Tr}\left(S_{11}^{B}(p) \gamma^{\sigma} S_{11}^{B}(k)\right)+\operatorname{Tr}\left(\gamma^{\sigma} S_{11}^{B}(p) S_{11}^{B}(k)\right)\right\} \\
& \left.+\frac{1}{4} g^{\mu \nu} g^{\alpha \beta} m^{2} \operatorname{Tr}\left(S_{11}^{B}(p) S_{11}^{B}(k)\right)\right\}+(\mu \leftrightarrow \nu)+(\alpha \leftrightarrow \beta)+(\mu \leftrightarrow \nu, \alpha \leftrightarrow \beta)
\end{aligned}
$$

where $S_{11}^{B}(p)$ is the 11-component of the momentum space real time free thermomagnetic Dirac propagator whose explicit form is given by $[64,65]$

$$
S_{11}^{B}(p)=\sum_{l=0}^{\infty}(-1)^{l} e^{-\alpha_{p}} \mathcal{D}_{l}(p) \tilde{D}_{11}\left(p_{\|}, m_{l}\right)
$$

in which $m_{l}=\sqrt{m^{2}+(2 l+1-2 s) e B}=\sqrt{m^{2}+2 l e B}$ (for Dirac field, $\left.s=1 / 2\right), \tilde{D}_{11}$ is defined in Eq. (A16) and $\mathcal{D}_{l}(p)$ is

$$
\mathcal{D}_{l}(p)=\left(\not \not_{\|}+m\right)\left[\left(\mathbb{1}+i \gamma^{1} \gamma^{2}\right) L_{l}\left(2 \alpha_{p}\right)-\left(\mathbb{1}-i \gamma^{1} \gamma^{2}\right) L_{l-1}\left(2 \alpha_{p}\right)\right]-4 \not p_{\perp} L_{l-1}^{1}\left(2 \alpha_{p}\right)
$$

with the convention $L_{-1}(z)=L_{-1}^{1}(z)=0$.

We now substitute Eq. (B16) into Eq. (B15) and get after some simplifications

$$
\left\langle\mathcal{T}_{C} T_{\text {Dirac }}^{\mu \nu}(x) T_{\text {Dirac }}^{\alpha \beta}(y)\right\rangle_{11}^{B}=-\iint \frac{d^{4} p}{(2 \pi)^{4}} \frac{d^{4} k}{(2 \pi)^{4}} e^{-i(x-y) \cdot(p-k)} \sum_{l=0}^{\infty} \sum_{n=0}^{\infty} \tilde{D}_{11}\left(p_{\|} ; m_{n}\right) \tilde{D}_{11}\left(k_{\|} ; m_{l}\right) \mathcal{N}_{l n ; \text { Dirac }}^{\mu \nu \alpha \beta}(k, p),
$$

where

$$
\begin{aligned}
\mathcal{N}_{l n ; \text { Dirac }}^{\mu \nu \alpha \beta}(k, p)= & -(-1)^{l+n} e^{-\alpha_{k}-\alpha_{p}}\left\{\frac{1}{16}\left(p^{\nu} k^{\beta}+k^{\nu} p^{\beta}+p^{\nu} p^{\beta}+k^{\nu} k^{\beta}\right) \operatorname{Tr}\left(\gamma^{\mu} \mathcal{D}_{n}(p) \gamma^{\alpha} \mathcal{D}_{l}(l)\right)\right. \\
& -\frac{1}{16} g^{\mu \nu}\left(p_{\sigma} k^{\beta}+k_{\sigma} p^{\beta}+p_{\sigma} p^{\beta}+k_{\sigma} k^{\beta}\right) \operatorname{Tr}\left(\gamma^{\sigma} \mathcal{D}_{n}(p) \gamma^{\alpha} \mathcal{D}_{l}(l)\right)+\frac{1}{8} g^{\mu \nu} m\left(p^{\beta}+k^{\beta}\right) \operatorname{Tr}\left(\mathcal{D}_{n}(p) \gamma^{\alpha} \mathcal{D}_{l}(l)\right) \\
& -\frac{1}{16} g^{\alpha \beta}\left(p^{\nu} k_{\sigma}+k^{\nu} p_{\sigma}+p^{\nu} p_{\sigma}+k^{\nu} k_{\sigma}\right) \operatorname{Tr}\left(\gamma^{\mu} \mathcal{D}_{n}(p) \gamma^{\sigma} \mathcal{D}_{l}(l)\right)+\frac{1}{8} g^{\alpha \beta} m\left(p^{\nu}+k^{\nu}\right) \operatorname{Tr}\left(\gamma^{\mu} \mathcal{D}_{n}(p) \mathcal{D}_{l}(l)\right) \\
& +\frac{1}{16} g^{\mu \nu} g^{\alpha \beta}\left(p_{\sigma} k_{\rho}+k_{\sigma} p_{\rho}+p_{\sigma} p_{\rho}+k_{\sigma} k_{\rho}\right) \operatorname{Tr}\left(\gamma^{\sigma} \mathcal{D}_{n}(p) \gamma^{\rho} \mathcal{D}_{l}(l)\right) \\
& -\frac{1}{8} g^{\mu \nu} g^{\alpha \beta} m\left(p_{\sigma}+k_{\sigma}\right)\left\{\operatorname{Tr}\left(\mathcal{D}_{n}(p) \gamma^{\sigma} \mathcal{D}_{l}(l)\right)+\operatorname{Tr}\left(\gamma^{\sigma} \mathcal{D}_{n}(p) \mathcal{D}_{l}(l)\right)\right\} \\
& \left.+\frac{1}{4} g^{\mu \nu} g^{\alpha \beta} m^{2} \operatorname{Tr}\left(\mathcal{D}_{n}(p) \mathcal{D}_{l}(l)\right)\right\}+(\mu \leftrightarrow \nu)+(\alpha \leftrightarrow \beta)+(\mu \leftrightarrow \nu, \alpha \leftrightarrow \beta) .
\end{aligned}
$$

In the calculation of viscous coefficients, we actually need the quantity $\mathcal{N}_{l n ; \text { Dirac }}^{\mu \nu \alpha \beta}(k, k)$, which is easily followed from Eq. (B19) as 


$$
\begin{aligned}
\mathcal{N}_{l n ; \text { Dirac }}^{\mu \nu \alpha \beta}(k, k)= & -\frac{1}{4}\left[\mathcal{T}_{l n}^{\mu \alpha}(k) k^{\nu} k^{\beta}-g^{\mu \nu}\left\{\mathcal{T}_{l n}^{\sigma \alpha}(k) k_{\sigma} k^{\beta}-m \mathcal{T}_{l n}^{\alpha}(k) k^{\beta}\right\}-g^{\alpha \beta}\left\{\mathcal{T}_{l n}^{\mu \sigma}(k) k_{\sigma} k^{\nu}-m \mathcal{T}_{n l}^{\mu}(k) k^{\nu}\right\}\right. \\
& \left.+g^{\mu \nu} g^{\alpha \beta}\left\{\mathcal{T}_{l n}^{\sigma \rho}(k) k_{\sigma} k_{\rho}-2 m \mathcal{T}_{l n}^{\sigma}(k) k_{\sigma}+m^{2} \mathcal{T}_{l n}(k)\right\}\right]+(\mu \leftrightarrow \nu)+(\alpha \leftrightarrow \beta)+(\mu \leftrightarrow \nu, \alpha \leftrightarrow \beta),
\end{aligned}
$$

where

$$
\begin{aligned}
\mathcal{T}_{l n}^{\mu \nu}(k)= & (-1)^{l+n} e^{-2 \alpha_{k}} \operatorname{Tr}\left[\gamma^{\mu} \mathcal{D}_{n}(k) \gamma^{\nu} \mathcal{D}_{l}(k)\right] \\
= & 8\left[8\left(2 k_{\perp}^{\mu} k_{\perp}^{\nu}-k_{\perp}^{2} g^{\mu \nu}\right) \mathcal{B}_{l n}\left(k_{\perp}^{2}\right)+\left\{2 k_{\|}^{\mu} k_{\|}^{\nu}-g_{\|}^{\mu \nu}\left(k_{\|}^{2}-m^{2}\right)\right\} \mathcal{C}_{l n}\left(k_{\perp}^{2}\right)\right. \\
& \left.+\left(k_{\|}^{2}-m^{2}\right) g_{\perp}^{\mu \nu} \mathcal{D}_{l n}\left(k_{\perp}^{2}\right)+2\left(k_{\|}^{\mu} k_{\perp}^{\nu}+k_{\perp}^{\mu} k_{\|}^{\nu}\right) \mathcal{E}_{l n}\left(k_{\perp}^{2}\right)\right] \\
\mathcal{T}_{l n}^{\mu}(k)= & (-1)^{l+n} e^{-2 \alpha_{k}} \operatorname{Tr}\left[\mathcal{D}_{n}(k) \gamma^{\mu} \mathcal{D}_{l}(k)\right]=16 m\left[k_{\|}^{\mu} \mathcal{C}_{l n}\left(k_{\perp}^{2}\right)+k_{\perp}^{\mu} \mathcal{D}_{l n}\left(k_{\perp}^{2}\right)\right], \\
\mathcal{T}_{l n}(k)= & (-1)^{l+n} e^{-2 \alpha_{k}} \operatorname{Tr}\left[\mathcal{D}_{n}(k) \mathcal{D}_{l}(k)\right]=8\left[8 k_{\perp}^{2} \mathcal{B}_{l n}\left(k_{\perp}^{2}\right)+\left(k_{\|}^{2}+m^{2}\right) \mathcal{C}_{l n}\left(k_{\perp}^{2}\right)\right]
\end{aligned}
$$

in which

$$
\begin{aligned}
& \mathcal{B}_{l n}\left(k_{\perp}^{2}\right)=(-1)^{l+n} e^{-2 \alpha_{k}} L_{l-1}^{1}\left(2 \alpha_{k}\right) L_{n-1}^{1}\left(2 \alpha_{k}\right), \\
& \mathcal{C}_{l n}\left(k_{\perp}^{2}\right)=(-1)^{l+n} e^{-2 \alpha_{k}}\left\{L_{l-1}\left(2 \alpha_{k}\right) L_{n-1}\left(2 \alpha_{k}\right)+L_{l}\left(2 \alpha_{k}\right) L_{n}\left(2 \alpha_{k}\right)\right\}, \\
& \mathcal{D}_{l n}\left(k_{\perp}^{2}\right)=(-1)^{l+n} e^{-2 \alpha_{k}}\left\{L_{l}\left(2 \alpha_{k}\right) L_{n-1}\left(2 \alpha_{k}\right)+L_{l-1}\left(2 \alpha_{k}\right) L_{n}\left(2 \alpha_{k}\right)\right\}, \\
& \mathcal{E}_{l n}\left(k_{\perp}^{2}\right)=(-1)^{l+n} e^{-2 \alpha_{k}}\left\{L_{l-1}\left(2 \alpha_{k}\right) L_{n-1}^{1}\left(2 \alpha_{k}\right)-L_{l}\left(2 \alpha_{k}\right) L_{n-1}^{1}\left(2 \alpha_{k}\right)+L_{l-1}^{1}\left(2 \alpha_{k}\right) L_{n-1}\left(2 \alpha_{k}\right)-L_{l-1}^{1}\left(2 \alpha_{k}\right) L_{n}\left(2 \alpha_{k}\right)\right\} .
\end{aligned}
$$

Substituting Eqs. (B21)-(B23) into Eq. (B20) and simplifying, we finally obtain

$$
\begin{aligned}
\mathcal{N}_{l n ; \text { scalar }}^{\mu \nu \alpha \beta}(k, k)= & -16 \mathcal{B}_{l n}\left(k_{\perp}^{2}\right)\left[k^{\nu} k^{\beta}\left(2 k_{\perp}^{\mu} k_{\perp}^{\alpha}-k_{\perp}^{2} g^{\mu \alpha}\right)-g^{\mu \nu} k^{\beta} k_{\perp}^{2}\left(k_{\perp}^{\alpha}-k_{\|}^{\alpha}\right)-g^{\alpha \beta} k^{\nu} k_{\perp}^{2}\left(k_{\perp}^{\mu}-k_{\|}^{\mu}\right)\right. \\
& \left.+g^{\mu \nu} g^{\alpha \beta} k_{\perp}^{2}\left(k_{\perp}^{2}-k_{\|}^{2}+m^{2}\right)\right]-2 \mathcal{C}_{l n}\left(k_{\perp}^{2}\right)\left[k^{\nu} k^{\beta}\left\{2 k_{\|}^{\mu} k_{\|}^{\alpha}-\left(k_{\|}^{2}-m^{2}\right) g_{\|}^{\mu \alpha}\right\}-\left(k_{\|}^{2}-m^{2}\right) g^{\mu \nu} k^{\beta} k_{\|}^{\alpha}\right. \\
& \left.-\left(k_{\|}^{2}-m^{2}\right) g^{\alpha \beta} k^{\nu} k_{\|}^{\mu}+g^{\mu \nu} g^{\alpha \beta}\left(k_{\|}^{2}-m^{2}\right)^{2}\right]-2 \mathcal{D}_{l n}\left(k_{\perp}^{2}\right)\left(k_{\|}^{2}-m^{2}\right)\left[k^{\nu} k^{\beta} g_{\perp}^{\mu \alpha}-g^{\mu \nu} k^{\beta} k_{\perp}^{\alpha}\right. \\
& \left.-g^{\alpha \beta} k^{\nu} k_{\perp}^{\mu}+g^{\mu \nu} g^{\alpha \beta} k_{\perp}^{2}\right]-4 \mathcal{E}_{l n}\left(k_{\perp}^{2}\right)\left[k^{\nu} k^{\beta}\left(k_{\|}^{\mu} k_{\perp}^{\alpha}+k_{\perp}^{\mu} k_{\|}^{\alpha}\right)-g^{\mu \nu} k^{\beta}\left\{\left(k_{\|}^{2}-m^{2}\right) k_{\perp}^{\alpha}+k_{\perp}^{2} k_{\|}^{\alpha}\right\}\right. \\
& \left.-g^{\alpha \beta} k^{\nu}\left\{\left(k_{\|}^{2}-m^{2}\right) k_{\perp}^{\mu}+k_{\perp}^{2} k_{\|}^{\mu}\right\}+2 g^{\mu \nu} g^{\alpha \beta} k_{\perp}^{2}\left(k_{\|}^{2}-m^{2}\right)\right]+(\mu \leftrightarrow \nu)+(\alpha \leftrightarrow \beta)+(\mu \leftrightarrow \nu, \alpha \leftrightarrow \beta) .
\end{aligned}
$$

\section{APPENDIX C: EXPLICIT ANALYTIC EXPRESSIONS OF $\mathcal{A}_{l n}^{(j)}, \mathcal{B}_{l n}^{(j)}, \ldots, \mathcal{E}_{l n}^{(j)}$}

Let us first note that, using the orthogonality of the Laguerre polynomials, the following integral identities can be derived:

$$
\begin{gathered}
\int \frac{d^{2} k_{\perp}}{(2 \pi)^{2}} e^{-2 \alpha_{k}} L_{l-1}^{1}\left(2 \alpha_{k}\right) L_{n-1}^{1}\left(2 \alpha_{k}\right) k_{\perp}^{4}=\frac{(e B)^{3}}{32 \pi} n l\left(2 \delta_{l-1}^{n-1}-\delta_{l-1}^{n}-\delta_{l-1}^{n-2}\right), \\
\int \frac{d^{2} k_{\perp}}{(2 \pi)^{2}} e^{-2 \alpha_{k}} L_{l-1}^{1}\left(2 \alpha_{k}\right) L_{n-1}^{1}\left(2 \alpha_{k}\right) k_{\perp}^{2}=-\frac{(e B)^{2}}{16 \pi} n \delta_{l-1}^{n-1}, \\
\int \frac{d^{2} k_{\perp}}{(2 \pi)^{2}} e^{-2 \alpha_{k}} L_{l-1}^{1}\left(2 \alpha_{k}\right) L_{n}\left(2 \alpha_{k}\right) k_{\perp}^{2}=-\frac{(e B)^{2}}{16 \pi} l\left(\delta_{l-1}^{n}-\delta_{l}^{n}\right), \\
\int \frac{d^{2} k_{\perp}}{(2 \pi)^{2}} e^{-2 \alpha_{k}} L_{l}\left(2 \alpha_{k}\right) L_{n}\left(2 \alpha_{k}\right) k_{\perp}^{4}=\frac{(e B)^{3}}{32 \pi}\left\{(2 l+1)^{2} \delta_{l}^{n}-(2 l+1) l \delta_{l}^{n+1}-(2 l+1) n \delta_{l}^{n-1}-(2 n+1) n \delta_{l+1}^{n}\right. \\
\left.+(l+1)^{2} \delta_{l+1}^{n+1}+(l+1) n \delta_{l+1}^{n-1}-(2 n+1) l \delta_{l-1}^{n}+(n+1) l \delta_{l-1}^{n+1}+l^{2} \delta_{l-1}^{n-1}\right\},
\end{gathered}
$$




$$
\begin{gathered}
\int \frac{d^{2} k_{\perp}}{(2 \pi)^{2}} e^{-2 \alpha_{k}} L_{l}\left(2 \alpha_{k}\right) L_{n}\left(2 \alpha_{k}\right) k_{\perp}^{2}=-\frac{(e B)^{2}}{16 \pi}\left\{(2 n+1) \delta_{l}^{n}-(n+1) \delta_{l}^{n+1}-n \delta_{l}^{n-1}\right\} \\
\int \frac{d^{2} k_{\perp}}{(2 \pi)^{2}} e^{-2 \alpha_{k}} L_{l}\left(2 \alpha_{k}\right) L_{n}\left(2 \alpha_{k}\right)=\frac{e B}{8 \pi} \delta_{l}^{n} .
\end{gathered}
$$

Now, using Eqs. (C1)-(C6), we perform the $d^{2} k_{\perp}$ integrals of Eqs. (78)-(82) and obtain

$$
\begin{gathered}
\mathcal{A}_{l n}^{(0)}=\frac{e B}{8 \pi} \delta_{l}^{n}, \\
\mathcal{A}_{l n}^{(2)}=-\frac{(e B)^{2}}{16 \pi}\left\{(2 n+1) \delta_{l}^{n}+(n+1) \delta_{l}^{n+1}+n \delta_{l}^{n-1}\right\}, \\
\mathcal{A}_{l n}^{(4)}=\frac{(e B)^{3}}{32 \pi}\left\{(2 l+1)^{2} \delta_{l}^{n}+(2 l+1) l \delta_{l}^{n+1}+(2 l+1) n \delta_{l}^{n-1}+(2 n+1) n \delta_{l+1}^{n}\right. \\
\left.+(l+1)^{2} \delta_{l+1}^{n+1}+(l+1) n \delta_{l+1}^{n-1}+(2 n+1) l \delta_{l-1}^{n}+(n+1) l \delta_{l-1}^{n+1}+l^{2} \delta_{l-1}^{n-1}\right\},
\end{gathered}
$$

$$
\begin{gathered}
\mathcal{B}_{l n}^{(2)}=-\frac{(e B)^{2}}{16 \pi} n \delta_{l-1}^{n-1}, \\
\mathcal{B}_{l n}^{(4)}=\frac{(e B)^{3}}{32 \pi} n l\left(2 \delta_{l-1}^{n-1}+\delta_{l-1}^{n}+\delta_{l-1}^{n-2}\right), \\
\mathcal{C}_{l n}^{(0)}=\frac{e B}{8 \pi}\left(\delta_{l}^{n}+\delta_{l-1}^{n-1}\right), \\
\mathcal{C}_{l n}^{(2)}=-\frac{(e B)^{2}}{16 \pi}\left\{(2 n+1) \delta_{l}^{n}+(n+1) \delta_{l}^{n+1}+n \delta_{l}^{n-1}\right. \\
\left.+(2 n-1) \delta_{l-1}^{n-1}+n \delta_{l-1}^{n}+(n-1) \delta_{l-1}^{n-2}\right\}, \\
\mathcal{D}_{l n}^{(0)}=-\frac{e B}{8 \pi}\left(\delta_{l}^{n-1}+\delta_{l-1}^{n}\right),
\end{gathered}
$$

$\mathcal{D}_{l n}^{(2)}=\frac{(e B)^{2}}{16 \pi}\left\{(2 n-1) \delta_{l}^{n-1}+n \delta_{l}^{n}+(n-1) \delta_{l}^{n-2}\right.$

$$
\left.+(2 n+1) \delta_{l-1}^{n}+(n+1) \delta_{l-1}^{n+1}+n \delta_{l-1}^{n-1}\right\},
$$

$\mathcal{E}_{l n}^{(2)}=-\frac{(e B)^{2}}{16 \pi}(l+n)\left(\delta_{l-1}^{n-1}+\delta_{l-1}^{n}+\delta_{l}^{n-1}+\delta_{l}^{n}\right)$.

It is important to note that the Kronecker delta function with a negative index is always zero (i.e., $\delta_{-1}^{-1}=0$ ), which follows from the convention of the Laguerre polynomials $L_{-1}(z)=L_{-1}^{1}(z)=0$ used in Eq. (B17).

Let us first consider the zero magnetic field case. The canonical partition function reads [66]

$$
\ln \mathcal{Z}=-V g \int \frac{d^{3} k}{(2 \pi)^{3}} a \ln \left(1-a e^{-\omega_{k} / T}\right)
$$

where $g$ is the degeneracy factor. For the system of charged scalar bosons $g=2$, whereas for the system of charged Dirac fermions $g=4$. All the thermodynamic quantities of interest can be derived from the partition function. The pressure is

$$
P=\frac{T}{V} \ln \mathcal{Z}=g \int \frac{d^{3} k}{(2 \pi)^{3}} \frac{\vec{k}^{2}}{3 \omega_{k}} f_{a}\left(\omega_{k}\right)
$$

where $f_{a}(x)=\left[e^{x / T}-a\right]^{-1}$ is the equilibrium thermal distribution (Bose-Einstein or Fermi-Dirac). Similarly, the energy density is given by

$$
\varepsilon=T\left(\frac{\partial P}{\partial T}\right)-P=g \int \frac{d^{3} k}{(2 \pi)^{3}} \omega_{k} f_{a}\left(\omega_{k}\right) .
$$

It is straightforward to obtain

$$
\theta=\left(\frac{\partial P}{\partial \varepsilon}\right)=\left(\frac{\partial P}{\partial T}\right) /\left(\frac{\partial \varepsilon}{\partial T}\right)
$$

where

\section{APPENDIX D: THERMODYNAMIC QUANTITIES}

In this appendix, we will derive the thermodynamic quantities $\theta=\left(\frac{\partial P}{\partial \varepsilon}\right)_{B}$ and $\phi=-B\left(\frac{\partial M}{\partial \varepsilon}\right)_{B}$ both at zero and nonzero external magnetic field, where $P$ is the longitudinal pressure, $\varepsilon$ is the energy density and $M$ is the magnetization.

$\left(\frac{\partial \varepsilon}{\partial T}\right)=\frac{1}{T^{2}} g \int \frac{d^{3} k}{(2 \pi)^{3}} \omega_{k}^{2} f_{a}\left(\omega_{k}\right)\left\{1+a f_{a}\left(\omega_{k}\right)\right\}$. 
Let us now switch on the external magnetic field. The canonical partition function for the charged particles now modifies to

$\ln \mathcal{Z}=-V \frac{e B}{2 \pi^{2}} \sum_{l=0}^{\infty} g_{l} \int_{0}^{\infty} \frac{d k_{z}}{(2 \pi)} a \ln \left(1-a e^{-\omega_{k l} / T}\right)$

where the degeneracy $g_{l}$ can now depend on the Landau level index $l$ as well. For the charged scalars $g_{l}=2$ whereas for the charged Dirac particles $g_{l}=2\left(2-\delta_{l}^{0}\right)$ implying that the lowest Landau level is spin nondegenerate. The longitudinal pressure and the energy density are given by

$P=\frac{T}{V} \ln \mathcal{Z}=T \frac{e B}{2 \pi^{2}} \sum_{l=0}^{\infty} g_{l} a \int_{0}^{\infty} d k_{z} \ln \left\{1+a f_{a}\left(\omega_{k l}\right)\right\}$,

$\varepsilon=T\left(\frac{\partial P}{\partial T}\right)_{B}-P=\frac{e B}{2 \pi^{2}} \sum_{l=0}^{\infty} g_{l} \int_{0}^{\infty} d k_{z} \omega_{k l} f_{a}\left(\omega_{k l}\right)$,

respectively, whereas the magnetization becomes

$$
\begin{aligned}
M & =\left(\frac{\partial P}{\partial B}\right)_{T} \\
& =\frac{1}{B}\left[P-\frac{(e B)^{2}}{4 \pi^{2}} \sum_{l=0}^{\infty} g_{l}(2 l+1-2 s) \int_{0}^{\infty} d k_{z} \frac{1}{\omega_{k l}} f_{a}\left(\omega_{k l}\right)\right] .
\end{aligned}
$$

The calculations of the quantities $\theta$ and $\phi$ at nonzero magnetic field are obtained from

$$
\theta=\left(\frac{\partial P}{\partial \varepsilon}\right)_{B}=\left(\frac{\partial P}{\partial T}\right)_{B} /\left(\frac{\partial \varepsilon}{\partial T}\right)_{B}
$$

$$
\phi=-B\left(\frac{\partial M}{\partial \varepsilon}\right)_{B}=-B\left(\frac{\partial M}{\partial T}\right)_{B} /\left(\frac{\partial \varepsilon}{\partial T}\right)_{B}
$$

where

$$
\begin{gathered}
\left(\frac{\partial P}{\partial T}\right)_{B}=\frac{\varepsilon+P}{T}, \\
\left(\frac{\partial \varepsilon}{\partial T}\right)_{B}=\frac{1}{T^{2}} \frac{e B}{2 \pi^{2}} \sum_{l=0}^{\infty} g_{l} \int_{0}^{\infty} d k_{z} \omega_{k l}^{2} f_{a}\left(\omega_{k l}\right)\left\{1+a f_{a}\left(\omega_{k l}\right)\right\},
\end{gathered}
$$

$$
\begin{aligned}
B\left(\frac{\partial M}{\partial T}\right)_{B}= & \left(\frac{\partial P}{\partial T}\right)_{B}-\frac{1}{T^{2}} \frac{(e B)^{2}}{4 \pi^{2}} \sum_{l=0}^{\infty} g_{l}(2 l+1-2 s) \\
& \times \int_{0}^{\infty} d k_{z} f_{a}\left(\omega_{k l}\right)\left\{1+a f_{a}\left(\omega_{k l}\right)\right\}
\end{aligned}
$$

[1] K. Tuchin, Adv. High Energy Phys. 2013, 490495 (2013).

[2] D. Kharzeev, J. Liao, S. Voloshin, and G. Wang, Prog. Part. Nucl. Phys. 88, 1 (2016).

[3] J. O. Andersen, W. R. Naylor, and A. Tranberg, Rev. Mod. Phys. 88, 025001 (2016).

[4] A. Bandyopadhyay and R. L. Farias, arXiv:2003.11054.

[5] R. Farias, K. Gomes, G. Krein, and M. Pinto, Phys. Rev. C 90, 025203 (2014).

[6] K. Tuchin, J. Phys. G 39, 025010 (2012).

[7] S. Li and H.-U. Yee, Phys. Rev. D 97, 056024 (2018).

[8] P. Mohanty, A. Dash, and V. Roy, Eur. Phys. J. A 55, 35 (2019).

[9] S. Ghosh, B. Chatterjee, P. Mohanty, A. Mukharjee, and H. Mishra, Phys. Rev. D 100, 034024 (2019).

[10] J. Dey, S. Satapathy, P. Murmu, and S. Ghosh, arXiv: 1907.11164.

[11] J. Dey, S. Satapathy, A. Mishra, S. Paul, and S. Ghosh, arXiv:1908.04335.
[12] A. Das, H. Mishra, and R. K. Mohapatra, Phys. Rev. D 100, 114004 (2019).

[13] M. Kurian, S. Mitra, S. Ghosh, and V. Chandra, Eur. Phys. J. C 79, 134 (2019).

[14] G. S. Denicol, X.-G. Huang, E. Molnár, G. M. Monteiro, H. Niemi, J. Noronha, D. H. Rischke, and Q. Wang, Phys. Rev. D 98, 076009 (2018).

[15] Z. Chen, C. Greiner, A. Huang, and Z. Xu, Phys. Rev. D 101, 056020 (2020).

[16] A. Jaiswal et al., Int. J. Mod. Phys. E 30, 2130001 (2021).

[17] A. Dash, S. Samanta, J. Dey, U. Gangopadhyaya, S. Ghosh, and V. Roy, Phys. Rev. D 102, 016016 (2020).

[18] K. Hattori, X.-G. Huang, D. H. Rischke, and D. Satow, Phys. Rev. D 96, 094009 (2017).

[19] N. Agasian, Phys. At. Nucl. 76, 1382 (2013).

[20] N. Agasian, JETP Lett. 95, 171 (2012).

[21] G. Kadam, Mod. Phys. Lett. A 30, 1550031 (2015).

[22] K. Hattori and D. Satow, Phys. Rev. D 94, 114032 (2016). 
[23] K. Hattori, S. Li, D. Satow, and H.-U. Yee, Phys. Rev. D 95, 076008 (2017).

[24] M. Kurian and V. Chandra, Phys. Rev. D 96, 114026 (2017).

[25] A. Harutyunyan and A. Sedrakian, Phys. Rev. C 94, 025805 (2016).

[26] S.-i. Nam, Phys. Rev. D 86, 033014 (2012).

[27] A. Das, H. Mishra, and R. K. Mohapatra, Phys. Rev. D 101, 034027 (2020).

[28] A. Das, H. Mishra, and R. K. Mohapatra, Phys. Rev. D 99, 094031 (2019).

[29] S. Ghosh, S. Ghosh, K. Goswami, S. Chakrabarty, and A. Goyal, Int. J. Mod. Phys. D 11, 843 (2002).

[30] S. Ghosh, A. Bandyopadhyay, R. L. Farias, J. Dey, and G. a. Krein, Phys. Rev. D 102, 114015 (2020).

[31] L. Thakur and P. Srivastava, Phys. Rev. D 100, 076016 (2019).

[32] S. Samanta, J. Dey, S. Satapathy, and S. Ghosh, arXiv: 2002.04434

[33] S. Rath and B. K. Patra, Eur. Phys. J. C 80, 747 (2020).

[34] P. Kalikotay, S. Ghosh, N. Chaudhuri, P. Roy, and S. Sarkar, Phys. Rev. D 102, 076007 (2020).

[35] E. Lifshitz and L. P. Pitaevski, Physical Kinetics (Elsevier, India, 1981).

[36] X.-G. Huang, A. Sedrakian, and D. H. Rischke, Ann. Phys. (Amsterdam) 326, 3075 (2011).

[37] R. Kubo, J. Phys. Soc. Jpn. 12, 570 (1957).

[38] S. Ghosh, Int. J. Mod. Phys. A 29, 1450054 (2014).

[39] S. Ghosh, Phys. Rev. C 90, 025202 (2014).

[40] D. Fernandez-Fraile and A. Gomez Nicola, Eur. Phys. J. C 62, 37 (2009).

[41] R. Lang, N. Kaiser, and W. Weise, Eur. Phys. J. A 48, 109 (2012).

[42] S. Jeon, Phys. Rev. D 52, 3591 (1995).

[43] S. Gavin, Nucl. Phys. A435, 826 (1985).

[44] P. Chakraborty and J. Kapusta, Phys. Rev. C 83, 014906 (2011).

[45] M. L. Bellac, Thermal Field Theory, Cambridge Monographs on Mathematical Physics (Cambridge University Press, Cambridge, England, 2011).

[46] S. Mallik and S. Sarkar, Hadrons at Finite Temperature (Cambridge University Press, Cambridge, England, 2016).
[47] W. Greiner and J. Reinhardt, Field Quantization (Springer, Germany, 1996).

[48] M. E. Peskin and D. V. Schroeder, An Introduction to Quantum Field Theory (Addison-Wesley, Reading, MA, 1995).

[49] A. Lahiri and P. B. Pal, A First Book of Quantum Field Theory (Narosa Publishing House, India, 2005).

[50] M. D. Schwartz, Quantum Field Theory and the Standard Model (Cambridge University Press, Cambridge, England, 2013).

[51] S. Ghosh, A. Mukherjee, P. Roy, and S. Sarkar, Phys. Rev. D 99, 096004 (2019).

[52] S. Ghosh, A. Mukherjee, M. Mandal, S. Sarkar, and P. Roy, Phys. Rev. D 96, 116020 (2017).

[53] S. Ghosh and V. Chandra, Phys. Rev. D 98, 076006 (2018).

[54] K. A. Mamo and H.-U. Yee, Phys. Rev. D 93, 065053 (2016).

[55] X.-G. Huang, M. Huang, D. H. Rischke, and A. Sedrakian, Phys. Rev. D 81, 045015 (2010).

[56] M. Hongo and K. Hattori, J. High Energy Phys. 02 (2021) 011.

[57] G. Sarwar, S. Chatterjee, and J.-e. Alam, J. Phys. G 44, 055101 (2017).

[58] S. I. Finazzo, R. Critelli, R. Rougemont, and J. Noronha, Phys. Rev. D 94, 054020 (2016); 96, 019903(E) (2017).

[59] R. Critelli, S. Finazzo, M. Zaniboni, and J. Noronha, Phys. Rev. D 90, 066006 (2014).

[60] S. Sharma, Adv. High Energy Phys. 2013, 452978 (2013).

[61] K. Saha, S. Upadhaya, and S. Ghosh, Mod. Phys. Lett. A 32, 1750018 (2017).

[62] D. Kharzeev and K. Tuchin, J. High Energy Phys. 09 (2008) 093.

[63] A. Ayala, A. Sanchez, G. Piccinelli, and S. Sahu, Phys. Rev. D 71, 023004 (2005).

[64] A. Ayala, A. Bashir, and S. Sahu, Phys. Rev. D 69, 045008 (2004).

[65] J. S. Schwinger, Phys. Rev. 82, 664 (1951).

[66] J. Kapusta and C. Gale, Finite-Temperature Field Theory: Principles and Applications, Cambridge Monographs on Mathematical Physics (Cambridge University Press, Cambridge, England, 2011). 